Pacific

Journal of

Mathematics

ON THE $\varepsilon$-REGULARIZATION OF THE MEAN CURVATURE FLOW PROBLEM WITH NEUMANN CONDITION

Yves Dumont 


\title{
ON THE $\varepsilon$-REGULARIZATION OF THE MEAN CURVATURE FLOW PROBLEM WITH NEUMANN CONDITION
}

\author{
Yves Dumont
}

\begin{abstract}
This paper deals with the study of the $\varepsilon$-regularization of the mean curvature equation with Neumann boundary conditions in one dimensional space. In particular, we measure the convergence of the regularized problem's solution to the viscosity solution of the mean curvature problem in appropriate topologies.
\end{abstract}

\section{Introduction.}

The topic of curve and surface evolution has recently generated great interest in the mathematical community because of its various applications such as crystal growth, flame propagation and image enhancement. The computation of such motion and its rigorous justification have proved to be difficult tasks. In particular, the motion of surface past singularities and changes of topological type has been the focus of extensive studies, see also [BSS, ES1, ESS].

1.1. The mean curvature flow problem. Consider the flow defined by

$$
\left\{\begin{aligned}
\frac{\partial u}{\partial t} & =|\nabla u| \nabla \cdot\left(\frac{\nabla u}{|\nabla u|}\right) & & \text { in } Q_{T}=\Omega \times(0, T), \\
\nabla u . \nu & =0 & & \text { on } S_{T}=\partial \Omega \times(0, T), \\
u(., 0) & =\phi & & \text { in } \bar{\Omega},
\end{aligned}\right.
$$

where $\Omega$ is a smooth bounded domain of $\mathbb{R}^{n}, \nu$ is the outer unit normal to $\partial \Omega$ and $\phi$ is a given data. Equation (1.1) is nonlinear, degenerated and undefined when $\nabla u(x, t)=0$. Evans and Spruck [ES1] and Chen et al. [CGG] circumvent these technical problems using the theory of viscosity solutions introduced by Crandall, Lions and Ishii [CLI]. In particular, they regularize the singular problem $(1.1)$ for $\varepsilon$ in $(0,1)$ by the partial differential equation

$$
\left\{\begin{aligned}
\frac{\partial u_{\varepsilon}}{\partial t}-\sqrt{\varepsilon^{2}+\left|\nabla u_{\varepsilon}\right|^{2}} \nabla \cdot\left(\frac{\nabla u_{\varepsilon}}{\sqrt{\varepsilon^{2}+\left|\nabla u_{\varepsilon}\right|^{2}}}\right) & =0 \text { in } Q_{T}, \\
\nabla u_{\varepsilon} . \nu & =0 \text { on } S_{T}, \\
u_{\varepsilon}(., 0) & =\phi \text { in } \bar{\Omega} .
\end{aligned}\right.
$$


Given a smooth function $\phi$ on $\Omega$ and under suitable assumptions on the boundary of the domain $\Omega$, the existence of a solution of (1.1) can be obtained using the same type of arguments as in $\left[\mathbf{E S 1 , ~ E S 4 ] ~ f o r ~} \Omega=\mathbb{R}^{n}\right.$, and in $[\mathbf{S Z}]$ for every smooth subset $\Omega$ of $\mathbb{R}^{n}$, first solving (1.2) and then passing to the limit as $\varepsilon$ goes to zero.

It is easy to prove that (1.2) is equivalent to

$$
\frac{\partial u_{\varepsilon}}{\partial t}(x, t)-\left(\delta_{i j}-\frac{\left(u_{\varepsilon}\right)_{x_{i}}\left(u_{\varepsilon}\right)_{x_{j}}}{\varepsilon^{2}+\left|\nabla u_{\varepsilon}\right|^{2}}\right)\left(u_{\varepsilon}\right)_{x_{i} x_{j}}=\frac{\partial u_{\varepsilon}}{\partial t}(x, t)-a_{\varepsilon}^{i j}\left(\nabla u_{\varepsilon}\right)_{x_{i} x_{j}},
$$

where

$$
a_{\varepsilon}^{i j}(p)=\delta_{i j}-\frac{p_{i} p_{j}}{\varepsilon^{2}+|p|^{2}} .
$$

Evans and Spruck [ES1] interpret (1.2) geometrically as follows. Assume $u_{\varepsilon}(x, t)$ to be a smooth solution of $(1.2)$ and write $y=\left(x, x_{n+1}\right) \in \mathbb{R}^{n+1}$ and define

$$
v_{\varepsilon}(x, t)=u_{\varepsilon}(x, t)-\varepsilon x_{n+1} .
$$

Then $\left|\nabla_{y} u_{\varepsilon}\right|=\varepsilon^{2}+\left|\nabla u_{\varepsilon}\right|$, and thus the PDE (1.2) becomes

$$
\left\{\begin{array}{rlrl}
\frac{\partial v_{\varepsilon}}{\partial t}(x, t)-\left(\delta_{i j}-\frac{\left(v_{\varepsilon}\right)_{i}\left(v_{\varepsilon}\right)_{j}}{\left|\nabla v_{\varepsilon}\right|^{2}}\right)\left(v_{\varepsilon}\right)_{y_{1} y_{j}}(x, t) & =0 & & \text { in } Q_{T} \\
\nabla v_{\varepsilon}(y, t) . \nu & =0 & & \text { on } S_{T} \\
v_{\varepsilon}(y, 0) & =\phi_{\varepsilon}(y) & \text { in } \bar{\Omega}
\end{array}\right.
$$

for $\phi_{\varepsilon}(y)=\phi(x)-\varepsilon x_{n+1}$. The PDE (1.5) means that each level set of $v_{\varepsilon}$ evolves according to its mean curvature. This is, in particular, the case for the zero level set

$$
\Gamma_{t}^{\varepsilon} \equiv\left\{y \in \bar{\Omega} \times \mathbb{R} \mid v_{\varepsilon}(y, t)=0\right\} .
$$

But according to (1.4), each $\Gamma_{t}^{\varepsilon}$ is a graph, i.e.,

$$
\Gamma_{t}^{\varepsilon} \equiv\left\{y=\left(x, x_{n+1}\right) \in \bar{\Omega} \times \mathbb{R} \mid x_{n+1}=\frac{1}{\varepsilon} u_{\varepsilon}(x, t)\right\},
$$

and Ecker and Huisken $[\mathbf{E H}]$ have shown that the evolution of an entire graph by mean curvature remains a smooth entire graph for all time. Therefore it seems to be interesting to use this property to compute numerically $u_{\varepsilon}$ as an approximation of the viscosity solution $u$ with respect to the parameter $\varepsilon$.

A corresponding finite element discretization of (1.2) with grid size $h$ is given by

$$
\int_{\Omega} \frac{u_{h t} \cdot \varphi_{h}+\nabla u_{h} \cdot \nabla \varphi_{h}}{\sqrt{\varepsilon^{2}+\left|\nabla u_{h}\right|^{2}}} d x=0, \quad \forall \varphi_{h} \in X_{h}, 0<t<T,
$$




$$
u_{h}(., 0)=\phi_{h},
$$

where $X_{h}$ is a suitable finite element space with grid size $h$. The regularization parameter is choosen according to the grid size $h$, e.g., $\varepsilon=h^{2}$. An additional time discretization leads to a nonlinear system which is linearized by a modified Newton's method. This gives a nonsymmetric linear system.

This algorithm is based on the analogous algorithm for the mean curvature flow for graphs $(\varepsilon=1)$ which was treated in $[\mathbf{W}]$ and for which asymptotic convergence has been proved in [DD].

Therefore, it would be interesting to know how fast $u_{\varepsilon}$ converges to $u$ as $\varepsilon$ goes to zero, i.e., find and prove an estimate of the norm $\left\|u_{\varepsilon}-u\right\| \leq C \varepsilon^{\alpha}$ with some power $\alpha>0$ for a suitable norm $\|\cdot\|$.

Numerical computations $[\mathbf{F r}]$ indicate a rate of convergence with respect to the square of the parameter $\varepsilon$, i.e., $\left\|u_{\varepsilon}-u\right\| \leq C \varepsilon^{2}$ with $C$ a positive constant.

The purpose of this article is to validate the numerical observations. Such estimates are provided in [D99] for the one dimensional problem with Dirichlet boundary conditions. We present here the extension of these results to Neumann boundary conditions.

1.2. The one dimensional problem. We now consider the nonlinear problem with nonhomogeneous Neumann boundary conditions in dimension one

$$
\left\{\begin{array}{rlrl}
\frac{\partial u_{\varepsilon}}{\partial t}(x, t)-\frac{\varepsilon^{2}}{\varepsilon^{2}+\left(\frac{\partial u_{\varepsilon}}{\partial x}(x, t)\right)^{2}} \frac{\partial^{2} u_{\varepsilon}}{\partial x^{2}}(x, t) & =0 & & \forall(x, t) \in Q_{T} \\
u_{\varepsilon}(x, 0) & =\phi(x) & & \forall x \in[0, l] \\
\frac{\partial u_{\varepsilon}}{\partial x}(0, t) & =\frac{\partial \phi}{\partial x}(0) & \forall t \in[0, T[ \\
\frac{\partial u_{\varepsilon}}{\partial x}(l, t) & =\frac{\partial \phi}{\partial x}(l) & \forall t \in[0, T[,
\end{array}\right.
$$

where $\left.Q_{T}=\right] 0, l[\times] 0, T[$.

For Neumann Condition, it is not possible to invoke the same type of computations used in [D99]. In particular, we will use in (1.6) an arctanformulation.

Our goal now is to find an asymptotic expansion of the solution $u_{\varepsilon}$ of (1.6) with respect to the regularization parameter $\varepsilon$ and to prove that this asymptotic expansion converges to the viscosity solution $\phi$ of (1.1) in some appropriate topology.

The paper is organized as follows. In $\S \mathbf{2}$, a short proof of the existence and uniqueness of a local solution $u_{\varepsilon}$ of (1.6) is given. In $\S \mathbf{3}$, a formal asymptotic expansion of the solution $u_{\varepsilon}$ in the powers of $\varepsilon$ is proposed. Then in $\S 4$ and $\S 5$ some estimates in appropriate topologies where the expansion of $u_{\varepsilon}$ is available are established:

- in $L^{2}\left(Q_{T}\right)$, when $\frac{\partial \phi}{\partial x}$ never reaches zero on $[0, l]$, 
- in appropriate weighted $L^{2}$-Sobolev spaces, when $\frac{\partial \phi}{\partial x}$ vanishes at a finite number of points in $[0, l]$.

\section{Local existence in Sobolev spaces.}

For every $T$ positive, we introduce the spaces $L^{\infty}\left(0, T ; L^{2}(0, l)\right)$ and $L^{2}\left(0, T ; H^{1}(0, l)\right)$ (see $\left.[\mathbf{L S U}]\right)$.

The Banach space $X=L^{\infty}\left(0, T ; L^{2}(0, l)\right) \cap L^{2}\left(0, T ; H^{1}(0, l)\right)$ is endowed with the norm

$$
\|u\|_{X}^{2}=\sup _{0 \leq t \leq T} \int_{0}^{l} u^{2}(x, t) d x+\int_{0}^{T} \int_{0}^{l}\left(\frac{\partial u}{\partial x}\right)^{2}(x, t) d x d t .
$$

The existence and uniqueness result reads as follows:

Theorem 2.1. Assume $\phi$ belongs to $H^{3}(0, l)$. Then there exist $T$ positive and a unique solution $u_{\varepsilon}$ of (1.6) which belongs to $X$. Moreover, $u_{\varepsilon}$ belongs to $L^{2}\left(0, T ; H^{3}(0, l)\right) \cap L^{\infty}\left(0, T ; H^{2}(0, l)\right)$ and $\frac{\partial u_{\varepsilon}}{\partial t}$ belongs to $L^{2}\left(0, T ; H^{2}(0, l)\right) \cap$ $L^{\infty}\left(0, T ; H^{1}(0, l)\right)$.

Sketch of the proof. We use Banach's fixed point Theorem as in [LSU]. Indeed, for every positive $\varepsilon$ and $M$, we introduce the set

$$
V_{\varepsilon, M}=\left\{\begin{array}{c}
u \in X \mid u(x, 0)=\phi(x), \frac{\partial u}{\partial x}(0, t)=\frac{\partial \phi}{\partial x}(0), \frac{\partial u}{\partial x}(l, t)=\frac{\partial \phi}{\partial x}(l), \\
u \in L^{2}\left(0, T ; H^{3}(0, l)\right) \cap L^{\infty}\left(0, T ; H^{2}(0, l)\right), \\
\frac{\partial u}{\partial t} \in L^{2}\left(0, T ; H^{2}(0, l)\right) \cap L^{\infty}\left(0, T ; H^{1}(0, l)\right), \\
\left\|\frac{\partial u}{\partial t}\right\|_{L^{\infty}\left(0, T ; H^{1}(0, l)\right)}^{2}+\left\|\frac{\partial u}{\partial t}\right\|_{L^{2}\left(0, T ; H^{2}(0, l)\right)}^{2}+ \\
+\|u\|_{L^{2}\left(0, T ; H^{3}(0, l)\right)}^{2}+\|u\|_{L^{\infty}\left(0, T ; H^{2}(0, l)\right)}^{2} \leq M \varepsilon^{2} .
\end{array}\right\}
$$

$V_{\varepsilon, M}$ is a closed and convex subset of $X$. We then consider the transformation $\mathbf{T}$ defined on $V_{\varepsilon, M}$ by: $w_{\varepsilon}=\mathbf{T} u$, where $w_{\varepsilon}$ is the solution of the following linear parabolic problem:

$$
\left\{\begin{aligned}
\left(1+\frac{1}{\varepsilon^{2}}\left(\frac{\partial u}{\partial x}(x, t)\right)^{2}\right) \frac{\partial w_{\varepsilon}}{\partial t}(x, t) & =\frac{\partial^{2} w_{\varepsilon}}{\partial x^{2}}(x, t)+\frac{\partial^{2} \phi}{\partial x^{2}}(x) & & \forall(x, t) \in Q_{T} \\
w_{\varepsilon}(x, 0) & =0 & & \forall x \in[0, l] \\
\frac{\partial w_{\varepsilon}}{\partial x}(0, t) & =0 & & \forall t \in[0, T] \\
\frac{\partial w_{\varepsilon}}{\partial x}(l, t) & =0 & & \forall t \in[0, T] .
\end{aligned}\right.
$$

For every $u$ in $V_{\varepsilon, M}$ we notice that

$$
\forall(x, t) \in Q_{T}: 0<\frac{\varepsilon^{2}}{\varepsilon^{2}+\left(\frac{\partial u}{\partial x}\right)^{2}(x, t)} \leq 1 .
$$

Hence from [LSU], there exists a unique solution $w_{\varepsilon}$ of (2.7) in $X$ satisfying, after some straightforward computations 


$$
\begin{aligned}
\| \frac{\partial w_{\varepsilon}}{\partial t} & \left\|_{L^{\infty}\left(0, T ; H^{1}(0, l)\right)}^{2}+\right\| \frac{\partial w_{\varepsilon}}{\partial t} \|_{L^{2}\left(0, T ; H^{2}(0, l)\right)}^{2} \\
& +\left\|w_{\varepsilon}\right\|_{L^{2}\left(0, T ; H^{3}(0, l)\right)}^{2}+\left\|w_{\varepsilon}\right\|_{L^{\infty}\left(0, T ; H^{2}(0, l)\right)}^{2} \leq C_{M}\|\phi\|_{H^{3}(0, l)}^{2} T,
\end{aligned}
$$

where $C_{M}$ is a constant independant of $\varepsilon$ but linearly dependant of $M$. If $T$ is sufficiently small, (2.8) implies that $w_{\varepsilon}$ belongs to $V_{\varepsilon, M}$. Moreover $\mathbf{T}$ is a contraction. Finally, Banach's fixed point Theorem implies the existence of a unique solution $u_{\varepsilon}$ of (1.6).

We are now looking for an asymptotic expansion in powers of the parameter $\varepsilon$ and estimates concerning $u_{\varepsilon}$ and its derivatives in order to justify this asymptotic expansion in adequate Sobolev spaces.

\section{The asymptotic expansion.}

A variational formulation of (1.6) is

$$
\left\{\begin{aligned}
\int_{0}^{T} \int_{0}^{l}\left(\varepsilon^{2}+\left(\frac{\partial u_{\varepsilon}}{\partial x}\right)^{2}\right) \frac{\partial u_{\varepsilon}}{\partial t} \varphi d x d t- & \\
-\varepsilon^{2} \int_{0}^{T} \int_{0}^{l} \frac{\partial^{2} u_{\varepsilon}}{\partial x^{2}} \frac{\partial \varphi}{\partial x} d x d t & =0, \forall \varphi \in L^{2}\left(0, T ; H^{1}(0, l)\right), \\
\frac{\partial u_{\varepsilon}}{\partial x}(0, t) & =\frac{\partial \phi}{\partial x}(0), \forall t \geq 0, \\
\frac{\partial u_{\varepsilon}}{\partial x}(l, t) & =\frac{\partial \phi}{\partial x}(l), \forall t \geq 0, \\
u_{\varepsilon}(x, 0)-\phi(x) & =0, \forall x \in[0, l] .
\end{aligned}\right.
$$

Let us suppose that $u_{\varepsilon}$ formally admits the following asymptotic expansion in powers of $\varepsilon$

$$
u_{\varepsilon}(x, t)=u_{0}(x, t)+\varepsilon u_{1}(x, t)+\varepsilon^{2} u_{2}(x, t)+\cdots .
$$

We replace $u_{\varepsilon}$ and its derivatives in (3.9) by their formal asymptotic expansions. We then deduce, when identifying the coefficients of $\varepsilon$-powers, that:

- The $\varepsilon^{0}$-term $u_{0}$ satisfies

$$
\left\{\begin{aligned}
\int_{0}^{T} \int_{0}^{l}\left(\frac{\partial u_{0}}{\partial x}\right)^{2} \frac{\partial u_{0}}{\partial t} \varphi d x d t & =0, & & \forall \varphi \in L^{2}\left(0, T ; H^{1}(0, l)\right), \\
u_{0}(x, 0) & =\phi(x), & & \forall x \in[0, l],
\end{aligned}\right.
$$

which implies $u_{0}(., t)=\phi$ in $L^{2}\left(Q_{T}\right)$.

- Then the $\varepsilon$-term $u_{1}$ satisfies

$$
\left\{\begin{aligned}
\int_{0}^{T} \int_{0}^{l}\left(\frac{\partial \phi}{\partial x}\right)^{2} \frac{\partial u_{1}}{\partial t} \varphi d x d t & =0 \quad \forall \varphi \in L^{2}\left(0, T ; H^{1}(0, l)\right), \\
u_{1}(x, 0) & =0, \quad \forall x \in[0, l]
\end{aligned}\right.
$$

which implies $\left(\frac{\partial \phi}{\partial x}\right)^{2}(x) u_{1}(x, t)=0$, almost everywhere in $Q_{T}$. 
- The $\varepsilon^{2}$-term $u_{2}$ satisfies

$$
\left\{\begin{array}{rlrl}
\int_{0}^{T} \int_{0}^{l}\left(\frac{\partial \phi}{\partial x}\right)^{4} \frac{\partial u_{2}}{\partial t} \varphi d x d t= & \int_{0}^{T} \int_{0}^{l}\left(\frac{\partial \phi}{\partial x}\right)^{2} \frac{\partial^{2} \phi}{\partial x^{2}} \varphi d x d t & & \\
& \forall \varphi \in L^{2}\left(0, T ; H^{1}(0, l)\right), & \\
u_{2}(x, 0)= & 0 & & \forall x \in[0, l], \\
\frac{\partial u_{2}}{\partial x}(l, t)= & 0 & & \forall t \geq 0, \\
\frac{\partial u_{2}}{\partial x}(l, t)= & 0 & & \forall t \geq 0,
\end{array}\right.
$$

which implies

$$
\left(\frac{\partial \phi}{\partial x}\right)^{2}(x) u_{2}(x, t)=t \frac{\partial^{2} \phi}{\partial x^{2}}(x), \text { almost everywhere in } Q_{T},
$$

when $\left|\frac{\partial \phi}{\partial x}(x)\right|>0$, for all $x$ in $[0, l]$, or

$\left(\frac{\partial \phi}{\partial x}(x)\right)^{4} u_{2}(x, t)=\left(\frac{\partial \phi}{\partial x}(x)\right)^{2} \frac{\partial^{2} \phi}{\partial x^{2}}(x) t$, almost everywhere in $Q_{T}$

when $\left|\frac{\partial \phi}{\partial x}(x)\right| \geq 0$, for all $x$ in $[0, l]$.

Finally, we have

$$
u_{\varepsilon}(x, t)=\phi(x)+\varepsilon^{2} u_{2}(x, t)+O\left(\varepsilon^{3}\right) .
$$

We now have to find the appropriate topology in which the asymptotic expansion (3.11) takes place.

Let us define $\omega_{\varepsilon}(x, t)=u_{\varepsilon}(x, t)-\phi(x)$. Then $\omega_{\varepsilon}$ satisfies the nonlinear problem

$$
\left\{\begin{array}{rlll}
\frac{\partial \omega_{\varepsilon}}{\partial t}-\frac{\varepsilon^{2}}{\varepsilon^{2}+\left(\frac{\partial \omega_{\varepsilon}}{\partial x}+\frac{\partial \phi}{\partial x}\right)^{2}}\left(\frac{\partial^{2} \omega_{\varepsilon}}{\partial x^{2}}+\frac{\partial^{2} \phi}{\partial x^{2}}\right) & =0 & \text { in } Q_{T} \\
\omega_{\varepsilon}(x, 0) & =0 & \forall x \in] 0, l[ \\
\frac{\partial \omega_{\varepsilon}}{\partial x}(0, t) & =0 & \forall t \in[0, T] \\
\frac{\partial \omega_{\varepsilon}}{\partial x}(l, t) & =0 & \forall t \in[0, T] .
\end{array}\right.
$$

A variational formulation of (3.12) is

$$
\begin{aligned}
\int_{0}^{T} \int_{0}^{l} \frac{\partial \omega_{\varepsilon}}{\partial t} \varphi d x d t+\frac{1}{\varepsilon^{2}} \int_{0}^{T} \int_{0}^{l}\left(\frac{\partial u_{\varepsilon}}{\partial x}\right)^{2} \frac{\partial \omega_{\varepsilon}}{\partial t} \varphi d x d t- & \\
& -\int_{0}^{T} \int_{0}^{l}\left(\frac{\partial^{2} \omega_{\varepsilon}}{\partial x^{2}}+\frac{\partial^{2} \phi}{\partial x^{2}}\right) \varphi d x d t=0 \quad \forall \varphi \in L^{2}\left(0, T ; H^{1}(0, l)\right) .
\end{aligned}
$$

In $\S \mathbf{4}$, we study the case $\left|\frac{\partial \phi}{\partial x}(x)\right|$ positive for all $x$ in $[0, l]$, and in $\S \mathbf{5}$ we study the case $\left|\frac{\partial \phi}{\partial x}(x)\right|$ nonnegative for all $x$ in $[0, l]$. 


\section{The first case.}

Using (3.13) with adequate test functions and after straightforward computations $[\mathbf{D}]$, we obtain:

\section{Lemma 4.2.}

1) The sequence $\left(\frac{1}{\varepsilon} \frac{\partial \omega_{\varepsilon}}{\partial x}\right)_{\varepsilon}$ is bounded in $L^{4}\left(Q_{T}\right) \cap L^{\infty}\left(0, T ; L^{4}(0, l)\right)$.

2) The sequence $\left(\frac{1}{\varepsilon^{2}} \frac{\partial \omega_{\varepsilon}}{\partial t}\right)_{\varepsilon}$ is bounded in $L^{2}\left(Q_{T}\right)$.

3) The sequence $\left(\frac{1}{\varepsilon^{2}} \omega_{\varepsilon}\right)_{\varepsilon}$ is bounded in $L^{2}\left(Q_{T}\right) \cap L^{\infty}\left(0, T ; L^{2}(0, l)\right)$.

From the previous Lemma, we deduce:

Theorem 4.3. If $\phi$ belongs to $H^{3}(0, l)$ and $\left|\frac{\partial \phi}{\partial x}\right|$ is positive in $[0, l]$, the sequence $\left(\frac{1}{\varepsilon^{2}} \omega_{\varepsilon}\right)_{\varepsilon}$ converges weakly in $L^{2}\left(Q_{T}\right)$ to the solution $u_{2}$ of $(3.10)$.

4.1. Further estimates and strong convergence. In order to make sure that the asymptotic expansion (3.11) is available in $L^{2}\left(Q_{T}\right)$, we define $\vartheta_{\varepsilon}=$ $u_{\varepsilon}-\phi-\varepsilon^{2} u_{2}$. Our purpose is to derive some estimates on $\vartheta_{\varepsilon}$ which satisfies the nonlinear problem

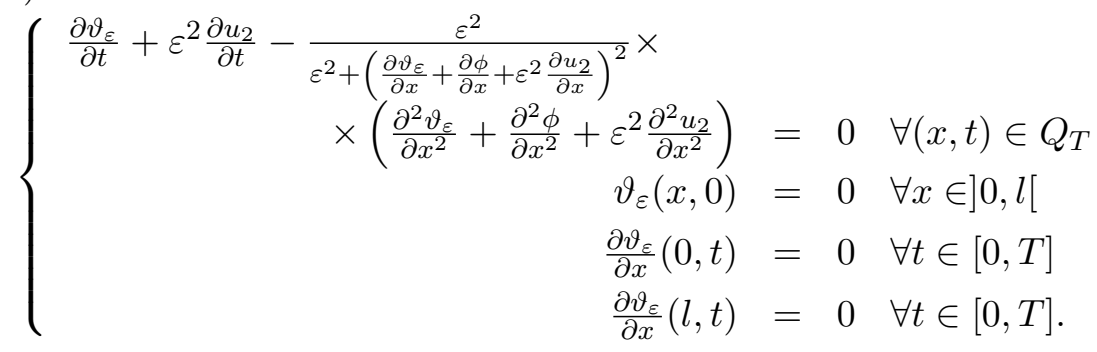

Using (4.14) with adequate test-functions and some straightforward computations $[\mathbf{D}]$, we deduce the following:

Theorem 4.4. Assuming that $\phi$ belongs to $H^{3}(0, l)$ and $\left|\frac{\partial \phi}{\partial x}\right|$ is positive in $[0, l]$, we have

1) the sequence $\left(\frac{1}{\varepsilon^{2}} \omega_{\varepsilon}\right)_{\varepsilon}$ converges strongly in $L^{2}\left(Q_{T}\right)$ to the solution $u_{2}$ of (3.10),

2) the asymptotic expansion (3.11) is available in $L^{2}\left(Q_{T}\right)$.

\section{The general case.}

We suppose that $\phi$ at least belongs to $H^{3}(0, l)$ and we allow $\frac{\partial \phi}{\partial x}$ to vanish at a finite number of points of $[0, l]$. We are first going to prove some intermediate a priori estimates on $\omega_{\varepsilon}=u_{\varepsilon}-\phi$, in order to obtain similar results to the ones obtained in the first case. The proofs are quite long and for reader's convenience we only develop some of them. 


\section{Lemma 5.5.}

1) Assuming that the solution $\omega_{\varepsilon}$ of (3.12) is smooth enough, it satisfies the following inequality

$$
\omega_{\varepsilon} \frac{\partial \omega_{\varepsilon}}{\partial t} \geq 0, \quad \text { a.e., in } Q_{T} .
$$

2) Assuming that the solution $u_{\varepsilon}$ of (1.6) is smooth enough, it satisfies the following inequalities

$$
\frac{\partial \phi}{\partial x} \frac{\partial u_{\varepsilon}}{\partial t} \geq 0, \quad \text { a.e., in } Q_{T} .
$$

Proof.

1. Define

$$
v_{\varepsilon}=\frac{\partial^{2} \phi}{\partial x^{2}} \frac{\partial u_{\varepsilon}}{\partial t}=\frac{\partial^{2} \phi}{\partial x^{2}} \frac{\partial w_{\varepsilon}}{\partial t}=\frac{\varepsilon^{2}}{\varepsilon^{2}+\left(\frac{\partial u_{\varepsilon}}{\partial x}\right)^{2}} \frac{\partial^{2} \phi}{\partial x^{2}} \frac{\partial^{2} u_{\varepsilon}}{\partial x^{2}} \quad \forall(x, t) \in Q_{T} .
$$

Since $u_{\varepsilon}(x, 0)=\phi(x)$, we get $v_{\varepsilon}(x, 0) \geq 0$ for all $x$ in $[0, l]$. We differentiate the previous equation with respect to $t$ (assuming that $u_{\varepsilon}$ is smooth enough). After some computations and multiplying by $\left(\frac{\partial^{2} \phi}{\partial x^{2}}\right)^{2}$, we obtain

$$
\begin{aligned}
& \left(\varepsilon^{2}+\left(\frac{\partial u_{\varepsilon}}{\partial x}\right)^{2}\right)\left(\frac{\partial^{2} \phi}{\partial x^{2}}\right)^{2} \frac{\partial v_{\varepsilon}}{\partial t}-\varepsilon^{2}\left(\varepsilon^{2}+\left(\frac{\partial u_{\varepsilon}}{\partial x}\right)^{2}\right)\left(\frac{\partial^{2} \phi}{\partial x^{2}}\right)^{2} \frac{\partial^{2} v_{\varepsilon}}{\partial x^{2}} \\
+ & 2 \varepsilon^{2}\left(\left(\varepsilon^{2}+\left(\frac{\partial u_{\varepsilon}}{\partial x}\right)^{2}\right) \frac{\partial^{2} \phi}{\partial x^{2}} \frac{\partial^{3} \phi}{\partial x^{3}}+\left(\frac{\partial^{2} \phi}{\partial x^{2}}\right)^{2} \frac{\partial u_{\varepsilon}}{\partial x} \frac{\partial^{2} u_{\varepsilon}}{\partial x^{2}}\right) \frac{\partial v_{\varepsilon}}{\partial x} \\
= & \varepsilon^{2}\left(\varepsilon^{2}+\left(\frac{\partial u_{\varepsilon}}{\partial x}\right)^{2}\right)\left(2\left(\frac{\partial^{3} \phi}{\partial x^{3}}\right)^{2}-\frac{\partial^{4} \phi}{\partial x^{4}} \frac{\partial^{2} \phi}{\partial x^{2}}\right) v_{\varepsilon} \\
& +2 \varepsilon^{2} \frac{\partial^{3} \phi}{\partial x^{3}} \frac{\partial^{2} \phi}{\partial x^{2}} \frac{\partial u_{\varepsilon}}{\partial x} \frac{\partial^{2} u_{\varepsilon}}{\partial x^{2}} v_{\varepsilon} .
\end{aligned}
$$

Let $w_{\varepsilon \sigma}(x, t)=e^{\sigma t} v_{\varepsilon}(x, t)$, with $\sigma<0$. Then $w_{\varepsilon \sigma}$ is the solution of

$$
\begin{aligned}
& \left(\varepsilon^{2}+\left(\frac{\partial u_{\varepsilon}}{\partial x}\right)^{2}\right)\left(\frac{\partial^{2} \phi}{\partial x^{2}}\right)^{2}\left(\frac{\partial w_{\varepsilon \sigma}}{\partial t}-\varepsilon^{2} \frac{\partial^{2} w_{\varepsilon \sigma}}{\partial x^{2}}\right) \\
+ & 2 \varepsilon^{2}\left(\left(\varepsilon^{2}+\left(\frac{\partial u_{\varepsilon}}{\partial x}\right)^{2}\right) \frac{\partial^{2} \phi}{\partial x^{2}} \frac{\partial^{3} \phi}{\partial x^{3}}+\left(\frac{\partial^{2} \phi}{\partial x^{2}}\right)^{2} \frac{\partial u_{\varepsilon}}{\partial x} \frac{\partial^{2} u_{\varepsilon}}{\partial x^{2}}\right) \frac{\partial w_{\varepsilon \sigma}}{\partial x} \\
= & \varepsilon^{2}\left(\varepsilon^{2}+\left(\frac{\partial u_{\varepsilon}}{\partial x}\right)^{2}\right)\left(2\left(\frac{\partial^{3} \phi}{\partial x^{3}}\right)^{2}-\frac{\partial^{4} \phi}{\partial x^{4}} \frac{\partial^{2} \phi}{\partial x^{2}}\right) w_{\varepsilon \sigma} \\
+ & \left(2 \varepsilon^{2} \frac{\partial^{3} \phi}{\partial x^{3}} \frac{\partial^{2} \phi}{\partial x^{2}} \frac{\partial u_{\varepsilon}}{\partial x} \frac{\partial^{2} u_{\varepsilon}}{\partial x^{2}}+\sigma\left(\varepsilon^{2}+\left(\frac{\partial u_{\varepsilon}}{\partial x}\right)^{2}\right)\left(\frac{\partial^{2} \phi}{\partial x^{2}}\right)^{2}\right) w_{\varepsilon \sigma} .
\end{aligned}
$$


Assuming that $w_{\varepsilon \sigma}$ is smooth enough, we choose $|\sigma|$ large enough such that the right hand side of the last equality is negative, at least at the points where $\frac{\partial^{2} \phi}{\partial x^{2}} \neq 0$. At $t=0, w_{\varepsilon \sigma}(x, 0)$ is nonnegative. Suppose that $w_{\varepsilon \sigma}$ becomes negative in $\overline{Q_{T}}$. Then it reaches a minimum $\beta$ at some point $\left(x_{\varepsilon \sigma}, t_{\varepsilon \sigma}\right)$. Due to the boundary conditions, we derive

$$
w_{\varepsilon \sigma}(0, t)=0=w_{\varepsilon \sigma}(l, t) \text { and } w_{\varepsilon \sigma}(x, 0) \geq 0,
$$

which imply that $\left(x_{\varepsilon \sigma}, t_{\varepsilon \sigma}\right)$ belongs to $\left.] 0, l[\times] 0, T\right]$. At this point, we have

$$
\begin{aligned}
\frac{\partial w_{\varepsilon \sigma}}{\partial t}\left(x_{\varepsilon \sigma}, t_{\varepsilon \sigma}\right) & \leq 0, \quad \frac{\partial w_{\varepsilon \sigma}}{\partial t}\left(x_{\varepsilon \sigma}, t_{\varepsilon \sigma}\right)=0, \\
w_{\varepsilon \sigma}\left(x_{\varepsilon \sigma}, t_{\varepsilon \sigma}\right) & =\beta<0, \quad \frac{\partial^{2} w_{\varepsilon \sigma}}{\partial x^{2}}\left(x_{\varepsilon \sigma}, t_{\varepsilon \sigma}\right) \geq 0 .
\end{aligned}
$$

This contradicts the fact that $w_{\varepsilon \sigma}$ satisfies the equation above. Then $w_{\varepsilon \sigma}$ is nonnegative in $Q_{T}$, hence $v_{\varepsilon}$, which implies

$$
\frac{\partial^{2} \phi}{\partial x^{2}} \frac{\partial w_{\varepsilon}}{\partial t}=\frac{\partial^{2} \phi}{\partial x^{2}} \frac{\partial u_{\varepsilon}}{\partial t} \geq 0, \text { in } Q_{T} .
$$

Since $w(x, 0)=0$, we deduce

$$
\frac{\partial^{2} \phi}{\partial x^{2}} w_{\varepsilon} \geq 0, \text { in } Q_{T} \Longrightarrow\left(\frac{\partial^{2} \phi}{\partial x^{2}}\right)^{2} w_{\varepsilon} \frac{\partial w_{\varepsilon}}{\partial t}, \text { in } Q_{T} .
$$

Finally, using the fact that $\left(\frac{\partial^{2} \phi}{\partial x^{2}}\right)^{2}$ vanishes at a finite number of points, we obtain the desired result.

2. The proof of this assertion is very similar to the previous one; it suffices to consider

$$
v_{\varepsilon}(x, t)=\frac{\partial \phi}{\partial x}(x) \frac{\partial u_{\varepsilon}}{\partial t}(x, t), \quad \forall(x, t) \in Q_{T} .
$$

5.1. Some estimates. Let us start the proofs of the estimates on $\left(\omega_{\varepsilon}\right)_{\varepsilon}$.

\section{Lemma 5.6.}

1) $\left(\omega_{\varepsilon}\right)_{\varepsilon}$ is bounded in $L^{2}\left(Q_{T}\right) \cap L^{\infty}\left(0, T ; L^{2}(0, l)\right)$

2) $\left(\frac{\partial \omega_{\varepsilon}}{\partial x}\right)_{\varepsilon}$ is bounded in $L^{2}\left(Q_{T}\right)$ and in $L^{\infty}\left(0, T ; L^{2}(0, l)\right)$

3) $\left(\frac{1}{\varepsilon} \frac{\partial u_{\varepsilon}}{\partial x} \frac{\partial \omega_{\varepsilon}}{\partial t}\right)_{\varepsilon}$ is bounded in $L^{2}\left(Q_{T}\right)$.

Proof.

1.2.3. We set $\varphi=\frac{\partial \omega_{\varepsilon}}{\partial t}$ in (3.13) and some straightforward computations imply the results. 


\section{Lemma 5.7.}

1) $\left(\frac{1}{\sqrt{\varepsilon}} \frac{\partial \phi}{\partial x} \omega_{\varepsilon}\right)_{\varepsilon}$ is bounded in $L^{2}\left(Q_{T}\right) \cap L^{\infty}\left(0, T ; L^{2}(0, l)\right)$ and in $L^{4}\left(Q_{T}\right) \cap$ $L^{\infty}\left(0, T ; L^{4}(0, l)\right)$.

2) $\left(\frac{1}{\varepsilon^{1 / 4}} \frac{\partial \phi}{\partial x} \frac{\partial \omega_{\varepsilon}}{\partial x}\right)_{\varepsilon}$ is bounded in $L^{2}\left(Q_{T}\right) \cap L^{\infty}\left(0, T ; L^{2}(0, l)\right)$.

3) $\left(\frac{1}{\varepsilon^{3 / 4}}\left(\frac{\partial \phi}{\partial x}\right)^{2} \frac{\partial \omega_{\varepsilon}}{\partial x} \omega_{\varepsilon}\right)_{\varepsilon}$ is bounded in $L^{2}\left(Q_{T}\right)$ and in $L^{\infty}\left(0, T ; L^{2}(0, l)\right)$.

4) $\left(\frac{1}{\varepsilon^{3 / 4}}\left(\frac{\partial \phi}{\partial x}\right)^{2} \frac{\partial \omega_{\varepsilon}}{\partial t} \omega_{\varepsilon}\right)_{\varepsilon}$ is bounded in $L^{2}\left(Q_{T}\right)$.

5) $\left(\frac{1}{\varepsilon^{3 / 4}} \frac{\partial u_{\varepsilon}}{\partial x}\left(\frac{\partial \phi}{\partial x}\right)^{2} \omega_{\varepsilon}\right)_{\varepsilon}$ is bounded in $L^{2}\left(Q_{T}\right)$ and in $L^{\infty}\left(0, T ; L^{2}(0, l)\right)$.

6) $\left(\frac{1}{\varepsilon^{3 / 4}}\left(\frac{\partial \phi}{\partial x}\right)^{3} \omega_{\varepsilon}\right)_{\varepsilon}$ is bounded in $L^{2}\left(Q_{T}\right) \cap L^{\infty}\left(0, T ; L^{2}(0, l)\right)$.

Proof. 1. Using the equality

$$
\frac{\varepsilon^{2}}{\varepsilon^{2}+\left(\frac{\partial u_{\varepsilon}}{\partial x}\right)^{2}} \frac{\partial^{2} u_{\varepsilon}}{\partial x^{2}}=\varepsilon \frac{\partial}{\partial x}\left(\arctan \left(\frac{1}{\varepsilon} \frac{\partial u_{\varepsilon}}{\partial x}\right)\right),
$$

a variational formulation of (3.12) can be written as

$$
\begin{array}{r}
\int_{0}^{T} \int_{0}^{l} \frac{\partial \omega_{\varepsilon}}{\partial t} \varphi d x d t=\varepsilon \int_{0}^{T} \int_{0}^{l} \frac{\partial}{\partial x}\left(\arctan \left(\frac{1}{\varepsilon} \frac{\partial u_{\varepsilon}}{\partial x}\right)\right) \varphi d x d t, \\
\forall \varphi \in L^{2}\left(0, T ; H^{1}(0, l)\right) .
\end{array}
$$

Setting $\varphi=\left(\frac{\partial \phi}{\partial x}\right)^{4} \omega_{\varepsilon}^{3}$ and integrating by parts with respect to $x$, we obtain

$$
\begin{aligned}
\frac{1}{4}\left\|\frac{\partial \phi}{\partial x} \omega_{\varepsilon}(., T)\right\|_{L^{4}(0, l)}^{4}= & \int_{0}^{T}\left[\varepsilon \arctan \left(\frac{1}{\varepsilon} \frac{\partial u_{\varepsilon}}{\partial x}\right)\left(\frac{\partial \phi}{\partial x}\right)^{4} \omega_{\varepsilon}^{3}\right]_{0}^{l} d t \\
& -4 \varepsilon \int_{0}^{T} \int_{0}^{l} \arctan \left(\frac{1}{\varepsilon} \frac{\partial u_{\varepsilon}}{\partial x}\right) \frac{\partial^{2} \phi}{\partial x^{2}}\left(\frac{\partial \phi}{\partial x}\right)^{3} \omega_{\varepsilon}^{3} d x d t \\
& -3 \varepsilon \int_{0}^{T} \int_{0}^{l} \arctan \left(\frac{1}{\varepsilon} \frac{\partial u_{\varepsilon}}{\partial x}\right)\left(\frac{\partial \phi}{\partial x}\right)^{4} \frac{\partial \omega_{\varepsilon}}{\partial x} \omega_{\varepsilon}^{2} d x d t .
\end{aligned}
$$

Since $\frac{\partial u_{\varepsilon}}{\partial x}(0, t)=\frac{\partial \phi}{\partial x}(0)$ and $\frac{\partial u_{\varepsilon}}{\partial x}(l, t)=\frac{\partial \phi}{\partial x}(l)$, we have

$$
\begin{aligned}
& {\left[\varepsilon \arctan \left(\frac{1}{\varepsilon} \frac{\partial u_{\varepsilon}}{\partial x}\right)\left(\frac{\partial \phi}{\partial x}\right)^{4} \omega_{\varepsilon}^{3}\right]_{0}^{l}} \\
& =\int_{0}^{l} \frac{\varepsilon^{2}}{\varepsilon^{2}+\left(\frac{\partial \phi}{\partial x}\right)^{2}} \frac{\partial^{2} \phi}{\partial x^{2}}\left(\frac{\partial \phi}{\partial x}\right)^{4} \omega_{\varepsilon}^{3} d x \\
& \quad+4 \varepsilon \int_{0}^{l} \arctan \left(\frac{1}{\varepsilon} \frac{\partial \phi}{\partial x}\right) \frac{\partial^{2} \phi}{\partial x^{2}}\left(\frac{\partial \phi}{\partial x}\right)^{3} \omega_{\varepsilon}^{3} d x
\end{aligned}
$$




$$
+3 \varepsilon \int_{0}^{l} \arctan \left(\frac{1}{\varepsilon} \frac{\partial \phi}{\partial x}\right)\left(\frac{\partial \phi}{\partial x}\right)^{4} \frac{\partial \omega_{\varepsilon}}{\partial x} \omega_{\varepsilon}^{2} d x .
$$

Then

$$
\begin{aligned}
\frac{1}{4} \| & \frac{\partial \phi}{\partial x} \omega_{\varepsilon}(., T) \|_{L^{4}(0, l)}^{4} \\
= & 4 \varepsilon \int_{0}^{T} \int_{0}^{l}\left(\arctan \left(\frac{1}{\varepsilon} \frac{\partial \phi}{\partial x}\right)-\arctan \left(\frac{1}{\varepsilon} \frac{\partial u_{\varepsilon}}{\partial x}\right)\right) \frac{\partial^{2} \phi}{\partial x^{2}}\left(\frac{\partial \phi}{\partial x}\right)^{3} \omega_{\varepsilon}^{3} d x d t \\
& +3 \varepsilon \int_{0}^{T} \int_{0}^{l}\left(\arctan \left(\frac{1}{\varepsilon} \frac{\partial \phi}{\partial x}\right)-\arctan \left(\frac{1}{\varepsilon} \frac{\partial u_{\varepsilon}}{\partial x}\right)\right)\left(\frac{\partial \phi}{\partial x}\right)^{4} \frac{\partial \omega_{\varepsilon}}{\partial x} \omega_{\varepsilon}^{2} d x d t \\
& +\int_{0}^{T} \int_{0}^{l} \frac{\varepsilon^{2}}{\varepsilon^{2}+\left(\frac{\partial \phi}{\partial x}\right)^{2}} \frac{\partial^{2} \phi}{\partial x^{2}}\left(\frac{\partial \phi}{\partial x}\right)^{4} \omega_{\varepsilon}^{3} d x d t .
\end{aligned}
$$

Since $-\frac{\pi}{2} \leq \arctan x \leq \frac{\pi}{2}$, we estimate

$$
\begin{aligned}
& 4 \varepsilon\left|\int_{0}^{T} \int_{0}^{l}\left(\arctan \left(\frac{1}{\varepsilon} \frac{\partial \phi}{\partial x}\right)-\arctan \left(\frac{1}{\varepsilon} \frac{\partial u_{\varepsilon}}{\partial x}\right)\right) \frac{\partial^{2} \phi}{\partial x^{2}}\left(\frac{\partial \phi}{\partial x}\right)^{3} \omega_{\varepsilon}^{3} d x d t\right| \\
& \leq C_{1} \varepsilon^{2}+\int_{0}^{T}\left\|\frac{\partial \phi}{\partial x} \omega_{\varepsilon}\right\|_{L^{4}(0, l)}^{4} d t
\end{aligned}
$$

and

$$
\begin{aligned}
& 3 \varepsilon\left|\int_{0}^{T} \int_{0}^{l}\left(\arctan \left(\frac{1}{\varepsilon} \frac{\partial \phi}{\partial x}\right)-\arctan \left(\frac{1}{\varepsilon} \frac{\partial u_{\varepsilon}}{\partial x}\right)\right)\left(\frac{\partial \phi}{\partial x}\right)^{4} \frac{\partial \omega_{\varepsilon}}{\partial x} \omega_{\varepsilon}^{2} d x d t\right| \\
& \leq C_{2} \varepsilon^{2}+\int_{0}^{T}\left\|\frac{\partial \phi}{\partial x} \omega_{\varepsilon}\right\|_{L^{4}(0, l)}^{4} d t
\end{aligned}
$$

due to Lemma 5.6 1).

Then, using the fact that $\frac{x^{2}}{\varepsilon^{2}+x^{2}} \leq 1$ for every real $x$, we compute

$$
\left|\int_{0}^{T} \int_{0}^{l} \frac{\varepsilon^{2}}{\varepsilon^{2}+\left(\frac{\partial \phi}{\partial x}\right)^{2}}\left(\frac{\partial \phi}{\partial x}\right)^{4} \frac{\partial^{2} \phi}{\partial x^{2}} \omega_{\varepsilon}^{3} d x d t\right| \leq C_{3} \varepsilon^{2}+\int_{0}^{T}\left\|\frac{\partial \phi}{\partial x} \omega_{\varepsilon}\right\|_{L^{4}(0, l)}^{4} d t .
$$

At last, we obtain

$$
\frac{1}{4}\left\|\frac{\partial \phi}{\partial x} \omega_{\varepsilon}(., T)\right\|_{L^{4}(0, l)}^{4} \leq C_{4} \varepsilon^{2}+3 \int_{0}^{T}\left\|\frac{\partial \phi}{\partial x} \omega_{\varepsilon}\right\|_{L^{4}(0, l)}^{4} d t,
$$

from which we deduce, using Gronwall's Lemma, that $\left(\frac{1}{\sqrt{\varepsilon}} \frac{\partial \phi}{\partial x} \omega_{\varepsilon}\right)$ is bounded in $L^{4}\left(Q_{T}\right) \cap L^{\infty}\left(0, T ; L^{4}(0, l)\right)$ and then in $L^{2}\left(Q_{T}\right) \cap L^{\infty}\left(0, T ; L^{2}(0, l)\right)$. 
2. Setting $\varphi=\left(\frac{\partial \phi}{\partial x}\right)^{2} \frac{\partial \omega_{\varepsilon}}{\partial t}$ in (3.13), we have

$$
\begin{aligned}
& \int_{0}^{T}\left\|\frac{\partial \phi}{\partial x} \frac{\partial \omega_{\varepsilon}}{\partial t}\right\|_{L^{2}(0, l)}^{2} d t+\frac{1}{\varepsilon^{2}} \int_{0}^{T}\left\|\frac{\partial \phi}{\partial x} \frac{\partial u_{\varepsilon}}{\partial x} \frac{\partial \omega_{\varepsilon}}{\partial t}\right\|_{L^{2}(0, l)}^{2} d t \\
& +\frac{1}{2}\left\|\frac{\partial \phi}{\partial x} \frac{\partial \omega_{\varepsilon}}{\partial x}(., T)\right\|_{L^{2}(0, l)}^{2}+2 \int_{0}^{T} \int_{0}^{l} \frac{\partial \omega_{\varepsilon}}{\partial x} \frac{\partial \phi}{\partial x} \frac{\partial^{2} \phi}{\partial x^{2}} \frac{\partial \omega_{\varepsilon}}{\partial t} d x d t \\
& =\int_{0}^{T} \int_{0}^{l}\left(\frac{\partial \phi}{\partial x}\right)^{2} \frac{\partial^{2} \phi}{\partial x^{2}} \frac{\partial \omega_{\varepsilon}}{\partial t} d x d t .
\end{aligned}
$$

We compute

$$
\begin{aligned}
2 \int_{0}^{T} \int_{0}^{l} \frac{\partial \omega_{\varepsilon}}{\partial x} \frac{\partial \phi}{\partial x} \frac{\partial^{2} \phi}{\partial x^{2}} \frac{\partial \omega_{\varepsilon}}{\partial t} d x d t= & 2 \int_{0}^{T} \int_{0}^{l} \frac{\partial u_{\varepsilon}}{\partial x} \frac{\partial \phi}{\partial x} \frac{\partial^{2} \phi}{\partial x^{2}} \frac{\partial \omega_{\varepsilon}}{\partial t} d x d t \\
& -2 \int_{0}^{T} \int_{0}^{l}\left(\frac{\partial \phi}{\partial x}\right)^{2} \frac{\partial^{2} \phi}{\partial x^{2}} \frac{\partial \omega_{\varepsilon}}{\partial t} d x d t .
\end{aligned}
$$

We combine the last term of the previous equality with the right hand side of (5.16) and we estimate these two terms in the following way

$$
\begin{aligned}
& 2\left|\int_{0}^{T} \int_{0}^{l} \frac{\partial u_{\varepsilon}}{\partial x} \frac{\partial \phi}{\partial x} \frac{\partial^{2} \phi}{\partial x^{2}} \frac{\partial \omega_{\varepsilon}}{\partial t} d x d t\right| \\
& \leq \frac{1}{2 \varepsilon^{2}} \int_{0}^{T} \|\left.\frac{\partial \phi}{\partial x} \frac{\partial u_{\varepsilon}}{\partial x} \frac{\partial \omega_{\varepsilon}}{\partial t}\right|_{L^{2}(0, l)} ^{2} d t+C_{1} \varepsilon^{2} \\
& 3\left|\int_{0}^{T} \int_{0}^{l}\left(\frac{\partial \phi}{\partial x}\right)^{2} \frac{\partial^{2} \phi}{\partial x^{2}} \frac{\partial \omega_{\varepsilon}}{\partial t} d x d t\right| \\
& =3\left|\int_{0}^{l}\left(\frac{\partial \phi}{\partial x}\right)^{2} \frac{\partial^{2} \phi}{\partial x^{2}} \omega_{\varepsilon}(., T) d x d t\right| \leq C_{2} \sqrt{\varepsilon}
\end{aligned}
$$

since $\phi$ belongs to $H^{3}(0, l)$ and $\left(\frac{1}{\sqrt{\varepsilon}} \frac{\partial \phi}{\partial x} \omega_{\varepsilon}\right)_{\varepsilon}$ is bounded in $L^{\infty}\left(0, T ; L^{2}(0, l)\right)$, Lemma 5.7 1). Finally, we have

$$
\begin{aligned}
\int_{0}^{T}\left\|\frac{\partial \phi}{\partial x} \frac{\partial \omega_{\varepsilon}}{\partial t}\right\|_{L^{2}(0, l)}^{2} d t+\frac{1}{2 \varepsilon^{2}} \int_{0}^{T} \| & \frac{\partial \phi}{\partial x} \frac{\partial u_{\varepsilon}}{\partial x} \frac{\partial \omega_{\varepsilon}}{\partial t} \|_{L^{2}(0, l)}^{2} d t \\
& +\frac{1}{2}\left\|\frac{\partial \phi}{\partial x} \frac{\partial \omega_{\varepsilon}}{\partial x}(., T)\right\|_{L^{2}(0, l)}^{2} \leq C_{3} \sqrt{\varepsilon}
\end{aligned}
$$

from which we deduce that $\left(\frac{1}{\varepsilon^{1 / 4}} \frac{\partial \phi}{\partial x} \frac{\partial \omega_{\varepsilon}}{\partial x}\right)$ is bounded in $L^{\infty}\left(0, T ; L^{2}(0, l)\right) \cap$ $L^{2}\left(Q_{T}\right)$. 
3. 4. Setting $\varphi=\left(\frac{\partial \phi}{\partial x}\right)^{4}\left(\omega_{\varepsilon}\right)^{2} \frac{\partial \omega_{\varepsilon}}{\partial t}$ in (3.13), we have

$$
\begin{aligned}
& \int_{0}^{T}\left\|\left(\frac{\partial \phi}{\partial x}\right)^{2} \omega_{\varepsilon} \frac{\partial \omega_{\varepsilon}}{\partial t}\right\|_{L^{2}(0, l)}^{2} d t+\frac{1}{\varepsilon^{2}} \int_{0}^{T}\left\|\left(\frac{\partial \phi}{\partial x}\right)^{2} \omega_{\varepsilon} \frac{\partial u_{\varepsilon}}{\partial x} \frac{\partial \omega_{\varepsilon}}{\partial t}\right\|_{L^{2}(0, l)}^{2} d t \\
& \quad+2 \int_{0}^{T} \int_{0}^{l} \omega_{\varepsilon}\left(\frac{\partial \omega_{\varepsilon}}{\partial x}\right)^{2}\left(\frac{\partial \phi}{\partial x}\right)^{4} \frac{\partial \omega_{\varepsilon}}{\partial t} d x d t \\
& \quad+\int_{0}^{T} \int_{0}^{l} \frac{\partial \omega_{\varepsilon}}{\partial x}\left(\omega_{\varepsilon}\right)^{2}\left(\frac{\partial \phi}{\partial x}\right)^{4} \frac{\partial^{2} \omega_{\varepsilon}}{\partial x \partial t} d x d t \\
& \quad+4 \int_{0}^{T} \int_{0}^{l} \frac{\partial \omega_{\varepsilon}}{\partial x}\left(\omega_{\varepsilon}\right)^{2}\left(\frac{\partial \phi}{\partial x}\right)^{3} \frac{\partial^{2} \phi}{\partial x^{2}} \frac{\partial \omega_{\varepsilon}}{\partial t} d x d t \\
& =\int_{0}^{T} \int_{0}^{l}\left(\omega_{\varepsilon}\right)^{2}\left(\frac{\partial \phi}{\partial x}\right)^{4} \frac{\partial^{2} \phi}{\partial x^{2}} \frac{\partial \omega_{\varepsilon}}{\partial t} d x d t .
\end{aligned}
$$

We compute the third and fourth terms of (5.17) as

$$
\begin{aligned}
& \int_{0}^{T} \int_{0}^{l} \frac{\partial \omega_{\varepsilon}}{\partial x}\left(\frac{\partial \phi}{\partial x}\right)^{4}\left(\omega_{\varepsilon}\right)^{2} \frac{\partial^{2} \omega_{\varepsilon}}{\partial x \partial t} d x d t \\
& \quad+2 \int_{0}^{T} \int_{0}^{l} \omega_{\varepsilon}\left(\frac{\partial \omega_{\varepsilon}}{\partial x}\right)^{2}\left(\frac{\partial \phi}{\partial x}\right)^{4} \frac{\partial \omega_{\varepsilon}}{\partial t} d x d t \\
& =\frac{1}{2}\left\|\frac{\partial \omega_{\varepsilon}}{\partial x}(., T)\left(\frac{\partial \phi}{\partial x}\right)^{2} \omega_{\varepsilon}(., T)\right\|_{L^{2}(0, l)}^{2} \\
& \quad+\int_{0}^{T} \int_{0}^{l}\left(\frac{\partial \omega_{\varepsilon}}{\partial x}\right)^{2}\left(\frac{\partial \phi}{\partial x}\right)^{4} \omega_{\varepsilon} \frac{\partial \omega_{\varepsilon}}{\partial t} d x d t .
\end{aligned}
$$

Using the fact that $\omega_{\varepsilon} \frac{\partial \omega_{\varepsilon}}{\partial t} \geq 0$, a.e. in $Q_{T}$, see Lemma 5.51 ), the last term of the previous equality is positive. Then, using the equality $\frac{\partial \omega_{\varepsilon}}{\partial x}=\frac{\partial u_{\varepsilon}}{\partial x}-\frac{\partial \phi}{\partial x}$, we compute the fifth term of (5.17)

$$
\begin{aligned}
& 4 \int_{0}^{T} \int_{0}^{l} \frac{\partial \omega_{\varepsilon}}{\partial x}\left(\omega_{\varepsilon}\right)^{2}\left(\frac{\partial \phi}{\partial x}\right)^{3} \frac{\partial^{2} \phi}{\partial x^{2}} \frac{\partial \omega_{\varepsilon}}{\partial t} d x d t \\
& =4 \int_{0}^{T} \int_{0}^{l} \frac{\partial u_{\varepsilon}}{\partial x}\left(\omega_{\varepsilon}\right)^{2}\left(\frac{\partial \phi}{\partial x}\right)^{3} \frac{\partial^{2} \phi}{\partial x^{2}} \frac{\partial \omega_{\varepsilon}}{\partial t} d x d t \\
& \quad-4 \int_{0}^{T} \int_{0}^{l}\left(\omega_{\varepsilon}\right)^{2}\left(\frac{\partial \phi}{\partial x}\right)^{4} \frac{\partial^{2} \phi}{\partial x^{2}} \frac{\partial \omega_{\varepsilon}}{\partial t} d x d t
\end{aligned}
$$


The previous terms can be combined with the right hand side of (5.17). Then, having in mind Lemmas 5.63 ) and 5.7 1), we estimate

$$
\begin{gathered}
4\left|\int_{0}^{T} \int_{0}^{l} \frac{\partial u_{\varepsilon}}{\partial x}\left(\omega_{\varepsilon}\right)^{2}\left(\frac{\partial \phi}{\partial x}\right)^{3} \frac{\partial^{2} \phi}{\partial x^{2}} \frac{\partial \omega_{\varepsilon}}{\partial t} d x d t\right| \leq C_{3} \varepsilon^{2}, \\
5\left|\int_{0}^{T} \int_{0}^{l}\left(\omega_{\varepsilon}\right)^{2}\left(\frac{\partial \phi}{\partial x}\right)^{4} \frac{\partial^{2} \phi}{\partial x^{2}} \frac{\partial \omega_{\varepsilon}}{\partial t} d x d t\right| \\
=\frac{5}{3}\left|\int_{0}^{l}\left(\omega_{\varepsilon}\right)^{3}(., T)\left(\frac{\partial \phi}{\partial x}\right)^{4} \frac{\partial^{2} \phi}{\partial x^{2}} d x\right| \\
\leq C_{4} \varepsilon \sqrt{\varepsilon} .
\end{gathered}
$$

Finally, we have

$$
\begin{array}{r}
\int_{0}^{T}\left\|\left(\frac{\partial \phi}{\partial x}\right)^{2} \omega_{\varepsilon} \frac{\partial \omega_{\varepsilon}}{\partial t}\right\|_{L^{2}(0, l)}^{2} d t+\frac{1}{\varepsilon^{2}} \int_{0}^{T}\left\|\omega_{\varepsilon}\left(\frac{\partial \phi}{\partial x}\right)^{2} \frac{\partial u_{\varepsilon}}{\partial x} \frac{\partial \omega_{\varepsilon}}{\partial t}\right\|_{L^{2}(0, l)}^{2} d t \\
+\frac{1}{2}\left\|\left(\frac{\partial \phi}{\partial x}\right)^{2} \frac{\partial \omega_{\varepsilon}}{\partial x}(., T) \omega_{\varepsilon}(., T)\right\|_{L^{2}(0, l)}^{2} \leq C_{5} \varepsilon \sqrt{\varepsilon}
\end{array}
$$

This implies that $\left(\frac{1}{\varepsilon^{3 / 4}}\left(\frac{\partial \phi}{\partial x}\right)^{2} \frac{\partial \omega_{\varepsilon}}{\partial x} \omega_{\varepsilon}\right)_{\varepsilon}$ is bounded in $L^{2}\left(Q_{T}\right) \cap L^{\infty}(0, T$; $\left.L^{2}(0, l)\right)$ and then $\left(\frac{1}{\varepsilon^{3 / 4}}\left(\frac{\partial \phi}{\partial x}\right)^{2} \frac{\partial \omega_{\varepsilon}}{\partial t} \omega_{\varepsilon}\right)_{\varepsilon}$ is bounded in $L^{2}\left(Q_{T}\right)$.

5. 6. Using the same test-function $\varphi=\left(\frac{\partial \phi}{\partial x}\right)^{4} \omega_{\varepsilon}^{2} \frac{\partial \omega_{\varepsilon}}{\partial t}$, we now compute

$$
\begin{aligned}
& \int_{0}^{T}\left\|\left(\frac{\partial \phi}{\partial x}\right)^{2} \omega_{\varepsilon} \frac{\partial \omega_{\varepsilon}}{\partial t}\right\|_{L^{2}(0, l)}^{2} d t+\frac{1}{\varepsilon^{2}} \int_{0}^{T}\left\|\frac{\partial u_{\varepsilon}}{\partial x}\left(\frac{\partial \phi}{\partial x}\right)^{2} \omega_{\varepsilon} \frac{\partial \omega_{\varepsilon}}{\partial t}\right\|_{L^{2}(0, l)}^{2} d t \\
& =-\frac{1}{2}\left\|\frac{\partial u_{\varepsilon}}{\partial x}(., T)\left(\frac{\partial \phi}{\partial x}\right)^{2} \omega_{\varepsilon}(., T)\right\|_{L^{2}(0, l)}^{2} \\
& \quad+\int_{0}^{T} \int_{0}^{l}\left(\frac{\partial u_{\varepsilon}}{\partial x}\right)^{2}\left(\frac{\partial \phi}{\partial x}\right)^{4} \omega_{\varepsilon} \frac{\partial \omega_{\varepsilon}}{\partial t} d x d t \\
& -4 \int_{0}^{T} \int_{0}^{l} \frac{\partial u_{\varepsilon}}{\partial x}\left(\frac{\partial \phi}{\partial x}\right)^{3} \frac{\partial^{2} \phi}{\partial x^{2}} \omega_{\varepsilon}^{2} \frac{\partial \omega_{\varepsilon}}{\partial t} d x d t \\
& \quad-2 \int_{0}^{T} \int_{0}^{l} \frac{\partial u_{\varepsilon}}{\partial x}\left(\frac{\partial \phi}{\partial x}\right)^{4} \frac{\partial \omega_{\varepsilon}}{\partial x} \omega_{\varepsilon} \frac{\partial \omega_{\varepsilon}}{\partial t} d x d t
\end{aligned}
$$




$$
+\int_{0}^{T}\left(\left(\frac{\partial \phi}{\partial x}(l)\right)^{5} \omega_{\varepsilon}^{2}(l, t) \frac{\partial \omega_{\varepsilon}}{\partial t}(l, t)-\left(\frac{\partial \phi}{\partial x}(0)\right)^{5} \omega_{\varepsilon}^{2}(0, t) \frac{\partial \omega_{\varepsilon}}{\partial t}(0, t)\right) d t,
$$

since $\frac{\partial^{2} \omega_{\varepsilon}}{\partial x \partial t}=\frac{\partial^{2} u_{\varepsilon}}{\partial x \partial t}, \frac{\partial u_{\varepsilon}}{\partial x}(l, t)=\frac{\partial \phi}{\partial x}(l), \frac{\partial u_{\varepsilon}}{\partial x}(0, t)=\frac{\partial \phi}{\partial x}(0)$. Using the fact that $\omega_{\varepsilon}(x, 0)=0$ and assuming that $\omega_{\varepsilon}$ is smooth enough, we have

$$
\begin{aligned}
\int_{0}^{T} & \left\|\left(\frac{\partial \phi}{\partial x}\right)^{2} \omega_{\varepsilon} \frac{\partial \omega_{\varepsilon}}{\partial t}\right\|_{L^{2}(0, l)}^{2} d t+\frac{1}{\varepsilon^{2}} \int_{0}^{T}\left\|\frac{\partial u_{\varepsilon}}{\partial x}\left(\frac{\partial \phi}{\partial x}\right)^{2} \omega_{\varepsilon} \frac{\partial \omega_{\varepsilon}}{\partial t}\right\|_{L^{2}(0, l)}^{2} d t \\
= & -\frac{1}{2}\left\|\frac{\partial u_{\varepsilon}}{\partial x}(., T)\left(\frac{\partial \phi}{\partial x}\right)^{2} \omega_{\varepsilon}(., T)\right\|_{L^{2}(0, l)}^{2} \\
& +\int_{0}^{T} \int_{0}^{l}\left(\frac{\partial u_{\varepsilon}}{\partial x}\right)^{2}\left(\frac{\partial \phi}{\partial x}\right)^{4} \omega_{\varepsilon} \frac{\partial \omega_{\varepsilon}}{\partial t} d x d t \\
& -4 \int_{0}^{T} \int_{0}^{l} \frac{\partial u_{\varepsilon}}{\partial x}\left(\frac{\partial \phi}{\partial x}\right)^{3} \frac{\partial^{2} \phi}{\partial x^{2}} \omega_{\varepsilon}^{2} \frac{\partial \omega_{\varepsilon}}{\partial t} d x d t \\
& -2 \int_{0}^{T} \int_{0}^{l} \frac{\partial u_{\varepsilon}}{\partial x}\left(\frac{\partial \phi}{\partial x}\right)^{4} \frac{\partial \omega_{\varepsilon}}{\partial x} \omega_{\varepsilon} \frac{\partial \omega_{\varepsilon}}{\partial t} d x d t \\
& +\frac{1}{3}\left(\left(\frac{\partial \phi}{\partial x}(l)\right)^{5} \omega_{\varepsilon}^{2}(l, T)-\left(\frac{\partial \phi}{\partial x}(0)\right)^{5} \omega_{\varepsilon}^{2}(0, T)\right) .
\end{aligned}
$$

We estimate the second term of (5.18)

$$
\begin{aligned}
\left|\int_{0}^{T} \int_{0}^{l}\left(\frac{\partial u_{\varepsilon}}{\partial x}\right)^{2}\left(\frac{\partial \phi}{\partial x}\right)^{4} \omega_{\varepsilon} \frac{\partial \omega_{\varepsilon}}{\partial t} d x d t\right| & \\
\leq & \frac{1}{4 \varepsilon^{2}} \int_{0}^{T}\left\|\frac{\partial u_{\varepsilon}}{\partial x}\left(\frac{\partial \phi}{\partial x}\right)^{2} \omega_{\varepsilon} \frac{\partial \omega_{\varepsilon}}{\partial t}\right\|_{L^{2}(0, l)}^{2} d t+C_{1} \varepsilon^{2},
\end{aligned}
$$

see Lemma 5.62 ). The third term of the right hand side of (5.18) is estimated as

$$
\begin{aligned}
4 \mid \int_{0}^{T} \int_{0}^{l} \frac{\partial u_{\varepsilon}}{\partial x}\left(\frac{\partial \phi}{\partial x}\right)^{3} & \frac{\partial^{2} \phi}{\partial x^{2}} \omega_{\varepsilon}^{2} \frac{\partial \omega_{\varepsilon}}{\partial t} d x d t \mid \\
& \leq \frac{1}{4 \varepsilon^{2}} \int_{0}^{T}\left\|\frac{\partial u_{\varepsilon}}{\partial x}\left(\frac{\partial \phi}{\partial x}\right)^{2} \omega_{\varepsilon} \frac{\partial \omega_{\varepsilon}}{\partial t}\right\|_{L^{2}(0, l)}^{2} d t+C_{2} \varepsilon^{3},
\end{aligned}
$$

since $\phi$ belongs to $H^{3}(0, L)$ and due to Lemma 5.71$)$. Then we estimate the fourth term of the right hand side of (5.18) 
$\left|2 \int_{0}^{T} \int_{0}^{l} \frac{\partial u_{\varepsilon}}{\partial x}\left(\frac{\partial \phi}{\partial x}\right)^{4} \frac{\partial \omega_{\varepsilon}}{\partial x} \omega_{\varepsilon} \frac{\partial \omega_{\varepsilon}}{\partial t} d x d t\right|$
$\leq \frac{1}{4 \varepsilon^{2}} \int_{0}^{T}\left\|\frac{\partial u_{\varepsilon}}{\partial x} \frac{\partial \phi}{\partial x} \omega_{\varepsilon} \frac{\partial \omega_{\varepsilon}}{\partial t}\right\|_{L^{2}(0, l)}^{2} d t+C_{3} \varepsilon^{2}$,

since $\left(\frac{\partial \omega_{\varepsilon}}{\partial x}\right)_{\varepsilon}$ is bounded in $L^{2}\left(Q_{T}\right)$, Lemma 5.62$)$. Using the equality $\frac{\partial \phi}{\partial x}=$ $\frac{\partial u_{\varepsilon}}{\partial x}-\frac{\partial \omega_{\varepsilon}}{\partial x}$, we rewrite the last term of the right hand side of (5.18)

$$
\begin{aligned}
& \frac{1}{3}\left(\left(\frac{\partial \phi}{\partial x}(l)\right)^{5} \omega_{\varepsilon}^{3}(l, T)-\left(\frac{\partial \phi}{\partial x}(0)\right)^{5} \omega_{\varepsilon}^{3}(0, t)\right) \\
& =\frac{5}{3} \int_{0}^{l}\left(\frac{\partial \phi}{\partial x}\right)^{5} \frac{\partial^{2} \phi}{\partial x^{2}} \omega_{\varepsilon}^{3}(., T) d x+\int_{0}^{l}\left(\frac{\partial \phi}{\partial x}\right)^{4} \frac{\partial u_{\varepsilon}}{\partial x}(., T) \frac{\partial \omega_{\varepsilon}}{\partial x}(., T) \omega_{\varepsilon}^{2}(., T) d x \\
& \quad-\int_{0}^{l}\left(\frac{\partial \phi}{\partial x}\right)^{4}\left(\frac{\partial \omega_{\varepsilon}}{\partial x}\right)^{2}(., T) \omega_{\varepsilon}^{2}(., T) d x .
\end{aligned}
$$

Using Lemma 5.7 1), we estimate

$$
\frac{5}{3}\left|\int_{0}^{l}\left(\frac{\partial \phi}{\partial x}\right)^{5} \frac{\partial^{2} \phi}{\partial x^{2}} \omega_{\varepsilon}^{3}(x, T) d x\right| \leq C_{4} \varepsilon \sqrt{\varepsilon},
$$

since $\left(\frac{1}{\sqrt{\varepsilon}} \frac{\partial \phi}{\partial x} \omega_{\varepsilon}\right)_{\varepsilon}$ is bounded in $L^{\infty}\left(0, T ; L^{4}(0, l)\right) \cap L^{\infty}\left(0, T ; L^{2}(0, l)\right)$. Then, having in mind Lemma 5.7 3), we deduce

$$
\begin{aligned}
& \left|\int_{0}^{l}\left(\frac{\partial \phi}{\partial x}\right)^{4}\left(\frac{\partial u_{\varepsilon}}{\partial x}(., T)-\frac{\partial \omega_{\varepsilon}}{\partial x}(., T)\right) \frac{\partial \omega_{\varepsilon}}{\partial x}(., T) \omega_{\varepsilon}^{2}(x, T) d x\right| \\
& \leq \frac{1}{4}\left\|\frac{\partial u_{\varepsilon}}{\partial x}(., T)\left(\frac{\partial \phi}{\partial x}\right)^{2} \omega_{\varepsilon}(., T)\right\|_{L^{2}(0, l)}^{2}+C_{5} \varepsilon \sqrt{\varepsilon}
\end{aligned}
$$

Finally, we have

$$
\begin{array}{r}
\int_{0}^{T}\left\|\left(\frac{\partial \phi}{\partial x}\right)^{2} \omega_{\varepsilon} \frac{\partial \omega_{\varepsilon}}{\partial t}\right\|_{L^{2}(0, l)}^{2} d t+\frac{1}{4 \varepsilon^{2}} \int_{0}^{T}\left\|\frac{\partial u_{\varepsilon}}{\partial x}\left(\frac{\partial \phi}{\partial x}\right)^{2} \omega_{\varepsilon} \frac{\partial \omega_{\varepsilon}}{\partial t}\right\|_{L^{2}(0, l)}^{2} d t \\
+\frac{1}{4}\left\|\frac{\partial u_{\varepsilon}}{\partial x}(., T)\left(\frac{\partial \phi}{\partial x}\right)^{2} \omega_{\varepsilon}(., T)\right\|_{L^{2}(0, l)}^{2} \leq C_{6} \varepsilon \sqrt{\varepsilon}
\end{array}
$$

We deduce that $\left(\frac{1}{\varepsilon^{3 / 4}} \frac{\partial u_{\varepsilon}}{\partial x}\left(\frac{\partial \phi}{\partial x}\right)^{2} \omega_{\varepsilon}\right)_{\varepsilon}$ is bounded in $L^{\infty}\left(0, T ; L^{2}(0, l)\right) \cap$ $L^{2}\left(Q_{T}\right)$. Using the previous estimate and Lemma 5.73$)$, we deduce that $\left(\frac{1}{\varepsilon^{3 / 4}}\left(\frac{\partial \phi}{\partial x}\right)^{3} \omega_{\varepsilon}\right)_{\varepsilon}$ is bounded in $L^{\infty}\left(0, T ; L^{2}(0, l)\right) \cap L^{2}\left(Q_{T}\right)$. 


\section{Lemma 5.8.}

1) $\left(\frac{1}{\varepsilon^{3 / 8}}\left(\frac{\partial \phi}{\partial x}\right)^{2} \frac{\partial \omega_{\varepsilon}}{\partial x}\right)$ is bounded in $L^{4}\left(Q_{T}\right)$.

2) $\left(\frac{1}{\varepsilon^{7 / 4}} \frac{\partial u_{\varepsilon}}{\partial x}\left(\frac{\partial \phi}{\partial x}\right)^{6} \frac{\partial \omega_{\varepsilon}}{\partial t}\right)_{\varepsilon}$ is bounded in $L^{2}\left(Q_{T}\right)$.

3) $\left(\frac{1}{\varepsilon^{3 / 4}}\left(\frac{\partial \phi}{\partial x}\right)^{6} \frac{\partial \omega_{\varepsilon}}{\partial t}\right)_{\varepsilon}$ is bounded in $L^{2}\left(Q_{T}\right)$.

4) $\left(\frac{1}{\varepsilon^{3 / 4}}\left(\frac{\partial \phi}{\partial x}\right)^{6} \frac{\partial \omega_{\varepsilon}}{\partial x}\right)_{\varepsilon}$ is bounded in $L^{\infty}\left(0, T ; L^{2}(0, l)\right) \cap L^{2}\left(Q_{T}\right)$.

Proof.

1. Setting $\varphi=\left(\frac{\partial \phi}{\partial x}\right)^{8}\left(\frac{\partial \omega_{\varepsilon}}{\partial x}\right)^{2} \omega_{\varepsilon}$ in (3.13) we obtain

$$
\begin{aligned}
& \int_{0}^{T} \int_{0}^{l}\left(\frac{\partial \phi}{\partial x}\right)^{8}\left(\frac{\partial \omega_{\varepsilon}}{\partial x}\right)^{2} \omega_{\varepsilon} \frac{\partial \omega_{\varepsilon}}{\partial t} d x d t \\
& \quad+\frac{1}{\varepsilon^{2}} \int_{0}^{T} \int_{0}^{l}\left(\frac{\partial \phi}{\partial x}\right)^{8}\left(\frac{\partial u_{\varepsilon}}{\partial x}\right)^{2}\left(\frac{\partial \omega_{\varepsilon}}{\partial x}\right)^{2} \omega_{\varepsilon} \frac{\partial \omega_{\varepsilon}}{\partial t} d x d t \\
& \quad-\int_{0}^{T} \int_{0}^{l} \frac{\partial^{2} \omega_{\varepsilon}}{\partial x^{2}}\left(\frac{\partial \phi}{\partial x}\right)^{8}\left(\frac{\partial \omega_{\varepsilon}}{\partial x}\right)^{2} \omega_{\varepsilon} d x d t \\
& =\int_{0}^{T} \int_{0}^{l} \frac{\partial^{2} \phi}{\partial x^{2}}\left(\frac{\partial \phi}{\partial x}\right)^{8}\left(\frac{\partial \omega_{\varepsilon}}{\partial x}\right)^{2} \omega_{\varepsilon} d x d t .
\end{aligned}
$$

Using the fact that $\omega_{\varepsilon} \frac{\partial \omega_{\varepsilon}}{\partial t}$ takes nonnegative values on $Q_{T}$, we observe that the first two terms of (5.19) are nonnegative. The fourth term of (5.19) leads to

$$
\begin{aligned}
& \left|\int_{0}^{T} \int_{0}^{l} \frac{\partial^{2} \phi}{\partial x^{2}}\left(\frac{\partial \phi}{\partial x}\right)^{8}\left(\frac{\partial \omega_{\varepsilon}}{\partial x}\right)^{2} \omega_{\varepsilon} d x d t\right| \\
& \leq \frac{1}{12} \int_{0}^{T}\left\|\left(\frac{\partial \phi}{\partial x}\right)^{2} \frac{\partial \omega_{\varepsilon}}{\partial x}\right\|_{L^{4}(0, l)}^{4} d t+C_{1} \varepsilon \sqrt{\varepsilon},
\end{aligned}
$$

due to Lemma 5.76 ). We compute the third term of (5.19) using an integration by parts and the equality $\frac{\partial \omega_{\varepsilon}}{\partial x}=\frac{\partial u_{\varepsilon}}{\partial x}-\frac{\partial \phi}{\partial x}$

$$
\begin{aligned}
& -\int_{0}^{T} \int_{0}^{l} \frac{\partial^{2} \omega_{\varepsilon}}{\partial x^{2}}\left(\frac{\partial \phi}{\partial x}\right)^{8}\left(\frac{\partial \omega_{\varepsilon}}{\partial x}\right)^{2} \omega_{\varepsilon} d x d t \\
= & \frac{1}{3} \int_{0}^{T}\left\|\left(\frac{\partial \phi}{\partial x}\right)^{2} \frac{\partial \omega_{\varepsilon}}{\partial x}\right\|_{L^{4}(0, l)}^{4} d t \\
& +\frac{8}{3} \int_{0}^{T} \int_{0}^{l} \frac{\partial^{2} \phi}{\partial x^{2}}\left(\frac{\partial \phi}{\partial x}\right)^{7}\left(\frac{\partial \omega_{\varepsilon}}{\partial x}\right)^{2} \frac{\partial u_{\varepsilon}}{\partial x} \omega_{\varepsilon} d x d t
\end{aligned}
$$




$$
-\frac{8}{3} \int_{0}^{T} \int_{0}^{l} \frac{\partial^{2} \phi}{\partial x^{2}}\left(\frac{\partial \phi}{\partial x}\right)^{8}\left(\frac{\partial \omega_{\varepsilon}}{\partial x}\right)^{2} \omega_{\varepsilon} d x d t
$$

Then we estimate

$$
\begin{aligned}
& \left|\frac{8}{3} \int_{0}^{T} \int_{0}^{l} \frac{\partial^{2} \phi}{\partial x^{2}}\left(\frac{\partial \phi}{\partial x}\right)^{7}\left(\frac{\partial \omega_{\varepsilon}}{\partial x}\right)^{2} \frac{\partial u_{\varepsilon}}{\partial x} \omega_{\varepsilon} d x d t\right| \\
& \leq \frac{1}{24} \int_{0}^{T}\left\|\left(\frac{\partial \phi}{\partial x}\right)^{2} \frac{\partial \omega_{\varepsilon}}{\partial x}\right\|_{L^{4}(0, l)}^{4} d t+C_{2} \varepsilon \sqrt{\varepsilon} \\
& \left|\frac{8}{3} \int_{0}^{T} \int_{0}^{l} \frac{\partial^{2} \phi}{\partial x^{2}}\left(\frac{\partial \phi}{\partial x}\right)^{8}\left(\frac{\partial \omega_{\varepsilon}}{\partial x}\right)^{2} \omega_{\varepsilon} d x d t\right| \\
& \leq \frac{1}{24} \int_{0}^{T}\left\|\left(\frac{\partial \phi}{\partial x}\right)^{2} \frac{\partial \omega_{\varepsilon}}{\partial x}\right\|_{L^{4}(0, l)}^{4} d t+C_{3} \varepsilon \sqrt{\varepsilon}
\end{aligned}
$$

since $\left(\frac{1}{\varepsilon^{3 / 4}}\left(\frac{\partial \phi}{\partial x}\right)^{3} \omega_{\varepsilon}\right)_{\varepsilon}$ and $\left(\frac{1}{\varepsilon^{3 / 4}} \frac{\partial u_{\varepsilon}}{\partial x}\left(\frac{\partial \phi}{\partial x}\right)^{2} \omega_{\varepsilon}\right)_{\varepsilon}$ are bounded in $L^{2}\left(Q_{T}\right)$, Lemma 5.75$), 6$ ). Finally we deduce

$$
\frac{1}{6} \int_{0}^{T}\left\|\left(\frac{\partial \phi}{\partial x}\right)^{2} \frac{\partial \omega_{\varepsilon}}{\partial x}\right\|_{L^{4}(0, l)}^{4} d t \leq C_{4} \varepsilon \sqrt{\varepsilon}
$$

which implies that $\left(\frac{1}{\varepsilon^{3 / 8}}\left(\frac{\partial \phi}{\partial x}\right)^{2} \frac{\partial \omega_{\varepsilon}}{\partial x}\right)$ is bounded in $L^{4}\left(Q_{T}\right)$.

2. 3. 4. Setting now $\varphi=\left(\frac{\partial \phi}{\partial x}\right)^{12} \frac{\partial \omega_{\varepsilon}}{\partial t}$ in (3.13), we have, integrating by parts the third integral of (3.13)

$$
\begin{aligned}
& \int_{0}^{T}\left\|\left(\frac{\partial \phi}{\partial x}\right)^{6} \frac{\partial \omega_{\varepsilon}}{\partial t}\right\|_{L^{2}(0, l)}^{2} d t+\frac{1}{\varepsilon^{2}} \int_{0}^{T}\left\|\frac{\partial u_{\varepsilon}}{\partial x}\left(\frac{\partial \phi}{\partial x}\right)^{6} \frac{\partial \omega_{\varepsilon}}{\partial t}\right\|_{L^{2}(0, l)}^{2} d t \\
& +12 \int_{0}^{T} \int_{0}^{l} \frac{\partial \omega_{\varepsilon}}{\partial x} \frac{\partial^{2} \phi}{\partial x^{2}}\left(\frac{\partial \phi}{\partial x}\right)^{11} \frac{\partial \omega_{\varepsilon}}{\partial t} d x d t+\int_{0}^{T} \int_{0}^{l} \frac{\partial \omega_{\varepsilon}}{\partial x}\left(\frac{\partial \phi}{\partial x}\right)^{12} \frac{\partial^{2} \omega_{\varepsilon}}{\partial t \partial x} d x d t \\
& =\int_{0}^{T} \int_{0}^{l} \frac{\partial^{2} \phi}{\partial x^{2}}\left(\frac{\partial \phi}{\partial x}\right)^{12} \frac{\partial \omega_{\varepsilon}}{\partial t} d x d t .
\end{aligned}
$$

Using the equality $\frac{\partial \omega_{\varepsilon}}{\partial x}=\frac{\partial u_{\varepsilon}}{\partial x}-\frac{\partial \phi}{\partial x}$, the previous equation can be rewritten in the following manner

$$
\int_{0}^{T}\left\|\left(\frac{\partial \phi}{\partial x}\right)^{6} \frac{\partial \omega_{\varepsilon}}{\partial t}\right\|_{L^{2}(0, l)}^{2} d t+\frac{1}{\varepsilon^{2}} \int_{0}^{T}\left\|\frac{\partial u_{\varepsilon}}{\partial x}\left(\frac{\partial \phi}{\partial x}\right)^{6} \frac{\partial \omega_{\varepsilon}}{\partial t}\right\|_{L^{2}(0, l)}^{2} d t
$$




$$
\begin{aligned}
& +12 \int_{0}^{T} \int_{0}^{l} \frac{\partial u_{\varepsilon}}{\partial x} \frac{\partial^{2} \phi}{\partial x^{2}}\left(\frac{\partial \phi}{\partial x}\right)^{11} \frac{\partial \omega_{\varepsilon}}{\partial t} d x d t+\int_{0}^{T} \int_{0}^{l} \frac{\partial \omega_{\varepsilon}}{\partial x}\left(\frac{\partial \phi}{\partial x}\right)^{12} \frac{\partial^{2} \omega_{\varepsilon}}{\partial t \partial x} d x d t \\
= & 13 \int_{0}^{T} \int_{0}^{l} \frac{\partial^{2} \phi}{\partial x^{2}}\left(\frac{\partial \phi}{\partial x}\right)^{12} \frac{\partial \omega_{\varepsilon}}{\partial t} d x d t .
\end{aligned}
$$

Then we estimate the third term of (5.20)

$$
\begin{aligned}
& 12\left|\int_{0}^{T} \int_{0}^{l} \frac{\partial u_{\varepsilon}}{\partial x} \frac{\partial^{2} \phi}{\partial x^{2}}\left(\frac{\partial \phi}{\partial x}\right)^{11} \frac{\partial \omega_{\varepsilon}}{\partial t} d x d t\right| \\
& \leq \frac{1}{2 \varepsilon^{2}} \int_{0}^{T}\left\|\frac{\partial u_{\varepsilon}}{\partial x}\left(\frac{\partial \phi}{\partial x}\right)^{6} \frac{\partial \omega_{\varepsilon}}{\partial t}\right\|_{L^{2}(0, l)}^{2} d t+C \varepsilon^{2},
\end{aligned}
$$

since $\phi$ belongs to $H^{3}(0, l)$ and the fourth term of (5.20) gives

$$
\left|\int_{0}^{T} \int_{0}^{l} \frac{\partial \omega_{\varepsilon}}{\partial x}\left(\frac{\partial \phi}{\partial x}\right)^{12} \frac{\partial^{2} \omega_{\varepsilon}}{\partial t \partial x} d x d t\right|=\frac{1}{2}\left\|\left(\frac{\partial \phi}{\partial x}\right)^{6} \frac{\partial \omega_{\varepsilon}}{\partial x}(., T)\right\|_{L^{2}(0, l)}^{2} .
$$

The right hand side of (5.20) can be computed as follows

$$
\begin{aligned}
& 13 \int_{0}^{T} \int_{0}^{l}\left(\frac{\partial \phi}{\partial x}\right)^{12} \frac{\partial^{2} \phi}{\partial x^{2}} \frac{\partial \omega_{\varepsilon}}{\partial t} d x d t \\
& =13 \int_{0}^{T} \int_{0}^{l} \frac{\partial^{2} \phi}{\partial x^{2}} \frac{\partial u_{\varepsilon}}{\partial x}\left(\frac{\partial \phi}{\partial x}\right)^{11} \frac{\partial \omega_{\varepsilon}}{\partial t} d x d t \\
& \quad-13 \int_{0}^{T} \int_{0}^{l} \frac{\partial^{2} \phi}{\partial x^{2}} \frac{\partial u_{\varepsilon}}{\partial x} \frac{\partial \omega_{\varepsilon}}{\partial x}\left(\frac{\partial \phi}{\partial x}\right)^{10} \frac{\partial \omega_{\varepsilon}}{\partial t} d x d t \\
& \quad+13 \int_{0}^{T} \int_{0}^{l} \frac{\partial^{2} \phi}{\partial x^{2}}\left(\frac{\partial \omega_{\varepsilon}}{\partial x}\right)^{2}\left(\frac{\partial \phi}{\partial x}\right)^{10} \frac{\partial \omega_{\varepsilon}}{\partial t} d x d t .
\end{aligned}
$$

Then we estimate the different terms in the right hand side of (5.21)

$$
\begin{aligned}
& \left|13 \int_{0}^{T} \int_{0}^{l} \frac{\partial^{2} \phi}{\partial x^{2}} \frac{\partial u_{\varepsilon}}{\partial x}\left(\frac{\partial \phi}{\partial x}\right)^{11} \frac{\partial \omega_{\varepsilon}}{\partial t} d x d t\right| \\
& \leq \frac{1}{4 \varepsilon^{2}} \int_{0}^{T}\left\|\frac{\partial u_{\varepsilon}}{\partial x}\left(\frac{\partial \phi}{\partial x}\right)^{6} \frac{\partial \omega_{\varepsilon}}{\partial t}\right\|_{L^{2}(0, l)}^{2} d t+C_{1} \varepsilon^{2}, \\
& \left|13 \int_{0}^{T} \int_{0}^{l} \frac{\partial^{2} \phi}{\partial x^{2}} \frac{\partial u_{\varepsilon}}{\partial x} \frac{\partial \omega_{\varepsilon}}{\partial x}\left(\frac{\partial \phi}{\partial x}\right)^{10} \frac{\partial \omega_{\varepsilon}}{\partial t} d x d t\right| \\
& \leq \frac{1}{4 \varepsilon^{2}} \int_{0}^{T}\left\|\frac{\partial u_{\varepsilon}}{\partial x}\left(\frac{\partial \phi}{\partial x}\right)^{6} \frac{\partial \omega_{\varepsilon}}{\partial t}\right\|_{L^{2}(0, l)}^{2} d t+C_{2} \varepsilon^{2},
\end{aligned}
$$




$$
\begin{aligned}
& \left|13 \int_{0}^{T} \int_{0}^{l} \frac{\partial^{2} \phi}{\partial x^{2}}\left(\frac{\partial \omega_{\varepsilon}}{\partial x}\right)^{2}\left(\frac{\partial \phi}{\partial x}\right)^{10} \frac{\partial \omega_{\varepsilon}}{\partial t} d x d t\right| \\
& \leq \frac{1}{2} \int_{0}^{T}\left\|\left(\frac{\partial \phi}{\partial x}\right)^{6} \frac{\partial \omega_{\varepsilon}}{\partial t}\right\|_{L^{2}(0, l)}^{2} d t+C_{3} \varepsilon \sqrt{\varepsilon}
\end{aligned}
$$

since $\phi$ belongs to $H^{3}(0, L)$ and $\left(\frac{1}{\varepsilon^{3 / 8}}\left(\frac{\partial \phi}{\partial x}\right)^{2} \frac{\partial \omega_{\varepsilon}}{\partial x}\right)_{\varepsilon}$ is bounded in $L^{4}\left(Q_{T}\right)$, Lemma 5.81 ). Finally we have

$$
\begin{array}{r}
\int_{0}^{T}\left\|\left(\frac{\partial \phi}{\partial x}\right)^{6} \frac{\partial \omega_{\varepsilon}}{\partial t}\right\|_{L^{2}(0, l)}^{2} d t+\frac{1}{2 \varepsilon^{2}} \int_{0}^{T}\left\|\frac{\partial u_{\varepsilon}}{\partial x}\left(\frac{\partial \phi}{\partial x}\right)^{6} \frac{\partial \omega_{\varepsilon}}{\partial t}\right\|_{L^{2}(0, l)}^{2} d t \\
+\left\|\left(\frac{\partial \phi}{\partial x}\right)^{6} \frac{\partial \omega_{\varepsilon}}{\partial x}(., T)\right\|_{L^{2}(0, l)}^{2} \leq C_{4} \varepsilon \sqrt{\varepsilon}
\end{array}
$$

which implies that $\left(\frac{1}{\varepsilon^{7 / 4}}\left(\frac{\partial \phi}{\partial x}\right)^{6} \frac{\partial u_{\varepsilon}}{\partial x} \frac{\partial \omega_{\varepsilon}}{\partial t}\right)_{\varepsilon}$ and $\left(\frac{1}{\varepsilon^{3 / 4}}\left(\frac{\partial \phi}{\partial x}\right)^{6} \frac{\partial \omega_{\varepsilon}}{\partial t}\right)_{\varepsilon}$ are bounded in $L^{2}\left(Q_{T}\right)$ and $\left(\frac{1}{\varepsilon^{3 / 4}}\left(\frac{\partial \phi}{\partial x}\right)^{6} \frac{\partial \omega_{\varepsilon}}{\partial x}\right)_{\varepsilon}$ is bounded in $L^{\infty}\left(0, T ; L^{2}(0, l)\right) \cap$ $L^{2}\left(Q_{T}\right)$.

\section{Lemma 5.9.}

1) $\left(\frac{1}{\varepsilon^{3 / 4}}\left(\frac{\partial \phi}{\partial x}\right)^{3} \omega_{\varepsilon}\right)$ is bounded in $L^{4}\left(Q_{T}\right) \cap L^{\infty}\left(0, T ; L^{4}(0, l)\right)$.

2) $\left(\frac{1}{\varepsilon}\left(\frac{\partial \phi}{\partial x}\right)^{4} \frac{\partial \omega_{\varepsilon}}{\partial x} \omega_{\varepsilon}\right)_{\varepsilon}$ is bounded in $L^{2}\left(Q_{T}\right) \cap L^{\infty}\left(0, T ; L^{2}(0, l)\right)$.

3) $\left(\frac{1}{\varepsilon}\left(\frac{\partial \phi}{\partial x}\right)^{4} \frac{\partial \omega_{\varepsilon}}{\partial t} \omega_{\varepsilon}\right)_{\varepsilon}$ is bounded in $L^{2}\left(Q_{T}\right)$.

4) $\left(\frac{1}{\varepsilon} \frac{\partial u_{\varepsilon}}{\partial x}\left(\frac{\partial \phi}{\partial x}\right)^{4} \omega_{\varepsilon}\right)_{\varepsilon}$ is bounded in $L^{2}\left(Q_{T}\right) \cap L^{\infty}\left(0, T ; L^{2}(0, l)\right)$.

5) $\left(\frac{1}{\varepsilon}\left(\frac{\partial \phi}{\partial x}\right)^{5} \omega_{\varepsilon}\right)_{\varepsilon}$ is bounded in $L^{2}\left(Q_{T}\right) \cap L^{\infty}\left(0, T ; L^{2}(0, l)\right)$.

Proof.

1. Setting $\varphi=\left(\frac{\partial \phi}{\partial x}\right)^{12} \omega_{\varepsilon}^{3}$ in (5.15) and using the same type of computations as in Lemma 5.71 ), we obtain

$$
\left\|\left(\frac{\partial \phi}{\partial x}\right)^{3} \omega_{\varepsilon}(., T)\right\|_{L^{4}(0, l)}^{4} \leq C \varepsilon^{3}+\int_{0}^{T}\left\|\left(\frac{\partial \phi}{\partial x}\right)^{3} \omega_{\varepsilon}\right\|_{L^{4}(0, l)}^{4} d t,
$$


from which we deduce, using Gronwall's Lemma, that $\left(\frac{1}{\varepsilon^{3 / 4}}\left(\frac{\partial \phi}{\partial x}\right)^{3} \omega_{\varepsilon}\right)_{\varepsilon}$ is bounded in $L^{4}\left(Q_{T}\right) \cap L^{\infty}\left(0, T ; L^{4}(0, l)\right)$.

2. 3. Setting $\varphi=\left(\frac{\partial \phi}{\partial x}\right)^{8}\left(\omega_{\varepsilon}\right)^{2} \frac{\partial \omega_{\varepsilon}}{\partial t}$ in (3.13), and using the same type of computations as in Lemma 5.7 3), 4), we obtain

$$
\begin{array}{r}
\int_{0}^{T}\left\|\left(\frac{\partial \phi}{\partial x}\right)^{4} \omega_{\varepsilon} \frac{\partial \omega_{\varepsilon}}{\partial t}\right\|_{L^{2}(0, l)}^{2} \quad d t+\frac{1}{2 \varepsilon^{2}} \int_{0}^{T}\left\|\omega_{\varepsilon}\left(\frac{\partial \phi}{\partial x}\right)^{4} \frac{\partial u_{\varepsilon}}{\partial x} \frac{\partial \omega_{\varepsilon}}{\partial t}\right\|_{L^{2}(0, l)}^{2} d t \\
+\frac{1}{2}\left\|\left(\frac{\partial \phi}{\partial x}\right)^{4} \frac{\partial \omega_{\varepsilon}}{\partial x}(., T) \omega_{\varepsilon}(., T)\right\|_{L^{2}(0, l)}^{2} \leq C \varepsilon^{2} .
\end{array}
$$

This implies that $\left(\frac{1}{\varepsilon}\left(\frac{\partial \phi}{\partial x}\right)^{4} \frac{\partial \omega_{\varepsilon}}{\partial x} \omega_{\varepsilon}\right)_{\varepsilon}$ is bounded in $L^{\infty}\left(0, T ; L^{2}(0, l)\right) \cap$ $L^{2}\left(Q_{T}\right)$ and also that $\left(\frac{1}{\varepsilon}\left(\frac{\partial \phi}{\partial x}\right)^{4} \frac{\partial \omega_{\varepsilon}}{\partial t} \omega_{\varepsilon}\right)_{\varepsilon}$ is bounded in $L^{2}\left(Q_{T}\right)$.

4. 5. Using the same test-function $\varphi=\left(\frac{\partial \phi}{\partial x}\right)^{8} \omega_{\varepsilon}^{2} \frac{\partial \omega_{\varepsilon}}{\partial t}$, and using the same type of computations as in Lemma 5.75 ), 6), we prove that

$$
\begin{array}{r}
\int_{0}^{T}\left\|\left(\frac{\partial \phi}{\partial x}\right)^{4} \omega_{\varepsilon} \frac{\partial \omega_{\varepsilon}}{\partial t}\right\|_{L^{2}(0, l)}^{2} d t+\frac{1}{4 \varepsilon^{2}} \int_{0}^{T}\left\|\frac{\partial u_{\varepsilon}}{\partial x}\left(\frac{\partial \phi}{\partial x}\right)^{4} \omega_{\varepsilon} \frac{\partial \omega_{\varepsilon}}{\partial t}\right\|_{L^{2}(0, l)}^{2} d t \\
+\frac{1}{4}\left\|\frac{\partial u_{\varepsilon}}{\partial x}(., T)\left(\frac{\partial \phi}{\partial x}\right)^{4} \omega_{\varepsilon}(., T)\right\|_{L^{2}(0, l)}^{2} \leq C \varepsilon^{2} .
\end{array}
$$

Then, we deduce that $\left(\frac{1}{\varepsilon} \frac{\partial u_{\varepsilon}}{\partial x}\left(\frac{\partial \phi}{\partial x}\right)^{4} \omega_{\varepsilon}\right)_{\varepsilon}$ is bounded in $L^{\infty}\left(0, T ; L^{2}(0, l)\right) \cap$ $L^{2}\left(Q_{T}\right)$. This estimate with that of Lemma 5.92$)$ imply that $\left(\frac{1}{\varepsilon}\left(\frac{\partial \phi}{\partial x}\right)^{5} \omega_{\varepsilon}\right)_{\varepsilon}$ is bounded in $L^{\infty}\left(0, T ; L^{2}(0, l)\right) \cap L^{2}\left(Q_{T}\right)$.

\section{Lemma 5.10.}
1) $\left(\frac{1}{\sqrt{\varepsilon}}\left(\frac{\partial \phi}{\partial x}\right)^{3} \frac{\partial \omega_{\varepsilon}}{\partial x}\right)_{\varepsilon}$ is bounded in $L^{4}\left(Q_{T}\right)$.
2) $\left(\frac{1}{\varepsilon^{2}} \frac{\partial u_{\varepsilon}}{\partial x}\left(\frac{\partial \phi}{\partial x}\right)^{8} \frac{\partial \omega_{\varepsilon}}{\partial t}\right)_{\varepsilon}$ is bounded in $L^{2}\left(Q_{T}\right)$.
3) $\left(\frac{1}{\varepsilon}\left(\frac{\partial \phi}{\partial x}\right)^{8} \frac{\partial \omega_{\varepsilon}}{\partial t}\right)_{\varepsilon}$ is bounded in $L^{2}\left(Q_{T}\right)$.
4) $\left(\frac{1}{\varepsilon}\left(\frac{\partial \phi}{\partial x}\right)^{8} \frac{\partial \omega_{\varepsilon}}{\partial x}\right)_{\varepsilon}$ is bounded in $L^{\infty}\left(0, T ; L^{2}(0, l)\right) \cap L^{2}\left(Q_{T}\right)$. 
Proof.

1. Setting $\varphi=\left(\frac{\partial \phi}{\partial x}\right)^{12}\left(\frac{\partial \omega_{\varepsilon}}{\partial x}\right)^{2} \omega_{\varepsilon}$ in (3.13) and using the same type of computations as in Lemma 5.81 ), we prove the desired result.

2. 3. 4. Setting $\varphi=\left(\frac{\partial \phi}{\partial x}\right)^{16} \frac{\partial \omega_{\varepsilon}}{\partial t}$ in (3.13) and after some computations, we obtain

$$
\begin{aligned}
& \frac{1}{2} \int_{0}^{T}\left\|\left(\frac{\partial \phi}{\partial x}\right)^{8} \frac{\partial \omega_{\varepsilon}}{\partial t}\right\|_{L^{2}(0, l)}^{2} d t+\frac{1}{4 \varepsilon^{2}} \int_{0}^{T}\left\|\frac{\partial u_{\varepsilon}}{\partial x}\left(\frac{\partial \phi}{\partial x}\right)^{8} \frac{\partial \omega_{\varepsilon}}{\partial t}\right\|_{L^{2}(0, l)}^{2} d t \\
& +\frac{1}{2}\left\|\left(\frac{\partial \phi}{\partial x}\right)^{8} \frac{\partial \omega_{\varepsilon}}{\partial x}(., T)\right\|_{L^{2}(0, l)}^{2} \leq C_{5} \varepsilon^{2},
\end{aligned}
$$

which implies that $\left(\frac{1}{\varepsilon^{2}}\left(\frac{\partial \phi}{\partial x}\right)^{8} \frac{\partial u_{\varepsilon}}{\partial x} \frac{\partial \omega_{\varepsilon}}{\partial t}\right)_{\varepsilon}$ and $\left(\frac{1}{\varepsilon}\left(\frac{\partial \phi}{\partial x}\right)^{8} \frac{\partial \omega_{\varepsilon}}{\partial t}\right)_{\varepsilon}$ are bounded in $L^{2}\left(Q_{T}\right)$ and $\left(\frac{1}{\varepsilon}\left(\frac{\partial \phi}{\partial x}\right)^{8} \frac{\partial \omega_{\varepsilon}}{\partial x}\right)_{\varepsilon}$ is bounded in $L^{\infty}\left(0, T ; L^{2}(0, l)\right) \cap L^{2}\left(Q_{T}\right)$.

\section{Lemma 5.11.}

1) $\left(\frac{1}{\varepsilon}\left(\frac{\partial \phi}{\partial x}\right)^{4} \omega_{\varepsilon}\right)_{\varepsilon}$ is bounded in $L^{4}\left(Q_{T}\right) \cap L^{\infty}\left(0, T ; L^{4}(0, l)\right)$.

2) $\left(\frac{1}{\varepsilon \sqrt{\varepsilon}}\left(\frac{\partial \phi}{\partial x}\right)^{7} \frac{\partial \omega_{\varepsilon}}{\partial x} \omega_{\varepsilon}\right)_{\varepsilon}$ is bounded in $L^{2}\left(Q_{T}\right)$ and in $L^{\infty}\left(0, T ; L^{2}(0, l)\right)$.

3) $\left(\frac{1}{\varepsilon \sqrt{\varepsilon}}\left(\frac{\partial \phi}{\partial x}\right)^{7} \frac{\partial \omega_{\varepsilon}}{\partial t} \omega_{\varepsilon}\right)_{\varepsilon}$ is bounded in $L^{2}\left(Q_{T}\right)$.

4) $\left(\frac{1}{\varepsilon \sqrt{\varepsilon}} \frac{\partial u_{\varepsilon}}{\partial x}\left(\frac{\partial \phi}{\partial x}\right)^{7} \omega_{\varepsilon}\right)_{\varepsilon}$ is bounded in $L^{2}\left(Q_{T}\right) \cap L^{\infty}\left(0, T ; L^{2}(0, l)\right)$.

5) $\left(\frac{1}{\varepsilon \sqrt{\varepsilon}}\left(\frac{\partial \phi}{\partial x}\right)^{8} \omega_{\varepsilon}\right)_{\varepsilon}$ is bounded in $L^{2}\left(Q_{T}\right) \cap L^{\infty}\left(0, T ; L^{2}(0, l)\right)$.

Proof.

1. Setting $\varphi=\left(\frac{\partial \phi}{\partial x}\right)^{16} \omega_{\varepsilon}^{3}$ in (5.15) and using the same type of computations as in Lemma 5.71 ), we have

$$
\left\|\left(\frac{\partial \phi}{\partial x}\right)^{4} \omega_{\varepsilon}(., T)\right\|_{L^{4}(0, l)}^{4} \leq C \varepsilon^{4}+\int_{0}^{T}\left\|\left(\frac{\partial \phi}{\partial x}\right)^{4} \omega_{\varepsilon}\right\|_{L^{4}(0, l)}^{4} d t
$$

from which we deduce using Gronwall's Lemma that $\left(\frac{1}{\varepsilon}\left(\frac{\partial \phi}{\partial x}\right)^{4} \omega_{\varepsilon}\right)_{\varepsilon}$ is bounded in $L^{4}\left(Q_{T}\right) \cap L^{\infty}\left(0, T ; L^{4}(0, l)\right)$. 
2. 3. Setting $\varphi=\left(\frac{\partial \phi}{\partial x}\right)^{14}\left(\omega_{\varepsilon}\right)^{2} \frac{\partial \omega_{\varepsilon}}{\partial t}$ in (3.13), and using the same type of computations as in Lemma 5.7 3), 4), we have

$$
\begin{array}{r}
\int_{0}^{T}\left\|\left(\frac{\partial \phi}{\partial x}\right)^{7} \omega_{\varepsilon} \frac{\partial \omega_{\varepsilon}}{\partial t}\right\|_{L^{2}(0, l)}^{2} d t+\frac{1}{2 \varepsilon^{2}} \int_{0}^{T}\left\|\omega_{\varepsilon}\left(\frac{\partial \phi}{\partial x}\right)^{7} \frac{\partial u_{\varepsilon}}{\partial x} \frac{\partial \omega_{\varepsilon}}{\partial t}\right\|_{L^{2}(0, l)}^{2} d t \\
+\frac{1}{2}\left\|\left(\frac{\partial \phi}{\partial x}\right)^{7} \frac{\partial \omega_{\varepsilon}}{\partial x}(., T) \omega_{\varepsilon}(., T)\right\|_{L^{2}(0, l)}^{2} \leq C_{3} \varepsilon^{3} .
\end{array}
$$

This implies that $\left(\frac{1}{\varepsilon \sqrt{\varepsilon}}\left(\frac{\partial \phi}{\partial x}\right)^{7} \frac{\partial \omega_{\varepsilon}}{\partial x} \omega_{\varepsilon}\right)_{\varepsilon}$ is bounded in $L^{\infty}\left(0, T ; L^{2}(0, l)\right) \cap$ $L^{2}\left(Q_{T}\right)$ and also that $\left(\frac{1}{\varepsilon \sqrt{\varepsilon}}\left(\frac{\partial \phi}{\partial x}\right)^{7} \frac{\partial \omega_{\varepsilon}}{\partial t} \omega_{\varepsilon}\right)_{\varepsilon}$ is bounded in $L^{2}\left(Q_{T}\right)$.

4. 5. Using the same test-function $\varphi=\left(\frac{\partial \phi}{\partial x}\right)^{14} \omega_{\varepsilon}^{2} \frac{\partial \omega_{\varepsilon}}{\partial t}$, and using the same type of computations as in Lemma 5.75 ), 6), we obtain

$$
\begin{gathered}
\int_{0}^{T}\left\|\left(\frac{\partial \phi}{\partial x}\right)^{7} \omega_{\varepsilon} \frac{\partial \omega_{\varepsilon}}{\partial t}\right\|_{L^{2}(0, l)}^{2} d t+\frac{1}{2 \varepsilon^{2}} \int_{0}^{T}\left\|\frac{\partial u_{\varepsilon}}{\partial x}\left(\frac{\partial \phi}{\partial x}\right)^{7} \omega_{\varepsilon} \frac{\partial \omega_{\varepsilon}}{\partial t}\right\|_{L^{2}(0, l)}^{2} d t \\
+\frac{1}{4}\left\|\frac{\partial u_{\varepsilon}}{\partial x}(., T)\left(\frac{\partial \phi}{\partial x}\right)^{7} \omega_{\varepsilon}(., T)\right\|_{L^{2}(0, l)}^{2} \\
\leq C \int_{0}^{T}\left\|\frac{\partial u_{\varepsilon}}{\partial x}\left(\frac{\partial \phi}{\partial x}\right)^{7} \omega_{\varepsilon}\right\|_{L^{2}(0, l)}^{2} d t+C_{6} \varepsilon^{3} .
\end{gathered}
$$

We deduce that $\left(\frac{1}{\varepsilon \sqrt{\varepsilon}} \frac{\partial u_{\varepsilon}}{\partial x}\left(\frac{\partial \phi}{\partial x}\right)^{7} \omega_{\varepsilon}\right)_{\varepsilon}$ is bounded in $L^{\infty}\left(0, T ; L^{2}(0, l)\right) \cap$ $L^{2}\left(Q_{T}\right)$.

Moreover, using this estimate and Lemma 5.112 ), we deduce that the sequence $\left(\frac{1}{\varepsilon \sqrt{\varepsilon}}\left(\frac{\partial \phi}{\partial x}\right)^{8} \omega_{\varepsilon}\right)_{\varepsilon}$ is bounded in $L^{\infty}\left(0, T ; L^{2}(0, l)\right) \cap L^{2}\left(Q_{T}\right)$.

\section{Lemma 5.12.}

1) $\left(\frac{1}{\varepsilon^{3 / 4}}\left(\frac{\partial \phi}{\partial x}\right)^{4} \frac{\partial \omega_{\varepsilon}}{\partial x}\right)_{\varepsilon}$ is bounded in $L^{4}\left(Q_{T}\right)$.

2) $\left(\frac{1}{\varepsilon^{5 / 4}}\left(\frac{\partial \phi}{\partial x}\right)^{5} \omega_{\varepsilon}\right)_{\varepsilon}$ is bounded in $L^{\infty}\left(0, T ; L^{4}(0, l)\right) \cap L^{4}\left(Q_{T}\right)$.

3) $\left(\frac{1}{\varepsilon^{2}}\left(\frac{\partial \phi}{\partial x}\right)^{9} \frac{\partial \omega_{\varepsilon}}{\partial x} \omega_{\varepsilon}\right)_{\varepsilon}$ is bounded in $L^{2}\left(Q_{T}\right)$ and in $L^{\infty}\left(0, T ; L^{2}(0, l)\right)$.

4) $\left(\frac{1}{\varepsilon^{2}}\left(\frac{\partial \phi}{\partial x}\right)^{9} \frac{\partial \omega_{\varepsilon}}{\partial t} \omega_{\varepsilon}\right)_{\varepsilon}$ is bounded in $L^{2}\left(Q_{T}\right)$. 
5) $\left(\frac{1}{\varepsilon^{2}} \frac{\partial u_{\varepsilon}}{\partial x}\left(\frac{\partial \phi}{\partial x}\right)^{9} \omega_{\varepsilon}\right)_{\varepsilon}$ is bounded in $L^{2}\left(Q_{T}\right) \cap L^{\infty}\left(0, T ; L^{2}(0, l)\right)$.

6) $\left(\frac{1}{\varepsilon^{2}}\left(\frac{\partial \phi}{\partial x}\right)^{10} \omega_{\varepsilon}\right)_{\varepsilon}$ is bounded in $L^{2}\left(Q_{T}\right) \cap L^{\infty}\left(0, T ; L^{2}(0, l)\right)$.

Proof.

1. Setting $\varphi=\left(\frac{\partial \phi}{\partial x}\right)^{16}\left(\frac{\partial \omega_{\varepsilon}}{\partial x}\right)^{2} \omega_{\varepsilon}$ in (3.13) and using the same type of computations as in Lemma 5.81 ), we obtain the result.

2. Setting $\varphi=\left(\frac{\partial \phi}{\partial x}\right)^{20} \omega_{\varepsilon}^{3}$ in (5.15) and integrating by part with respect to $x$, we obtain

$$
\begin{aligned}
\frac{1}{4}\left\|\left(\frac{\partial \phi}{\partial x}\right)^{5} \omega_{\varepsilon}(., T)\right\|_{L^{4}(0, l)}^{4} \\
=\int_{0}^{T}\left[\varepsilon \arctan \left(\frac{1}{\varepsilon} \frac{\partial u_{\varepsilon}}{\partial x}\right)\left(\frac{\partial \phi}{\partial x}\right)^{20} \omega_{\varepsilon}^{3}\right]_{0}^{l} d t \\
-20 \varepsilon \int_{0}^{T} \int_{0}^{l} \arctan \left(\frac{1}{\varepsilon} \frac{\partial u_{\varepsilon}}{\partial x}\right) \frac{\partial^{2} \phi}{\partial x^{2}}\left(\frac{\partial \phi}{\partial x}\right)^{19} \omega_{\varepsilon}^{3} d x d t \\
-3 \varepsilon \int_{0}^{T} \int_{0}^{l} \arctan \left(\frac{1}{\varepsilon} \frac{\partial u_{\varepsilon}}{\partial x}\right)\left(\frac{\partial \phi}{\partial x}\right)^{20} \frac{\partial \omega_{\varepsilon}}{\partial x} \omega_{\varepsilon}^{2} d x d t .
\end{aligned}
$$

Since $\frac{\partial u_{\varepsilon}}{\partial x}(0, t)=\frac{\partial \phi}{\partial x}(0)$ and $\frac{\partial u_{\varepsilon}}{\partial x}(l, t)=\frac{\partial \phi}{\partial x}(l)$, we compute

$$
\begin{aligned}
& {\left[\varepsilon \arctan \left(\frac{1}{\varepsilon} \frac{\partial u_{\varepsilon}}{\partial x}\right)\left(\frac{\partial \phi}{\partial x}\right)^{20} \omega_{\varepsilon}^{3}\right]_{0}^{l}} \\
& =20 \varepsilon \int_{0}^{l} \arctan \left(\frac{1}{\varepsilon} \frac{\partial \phi}{\partial x}\right) \frac{\partial^{2} \phi}{\partial x^{2}}\left(\frac{\partial \phi}{\partial x}\right)^{19} \omega_{\varepsilon}^{3} d x \\
& \quad+\int_{0}^{l} \frac{\varepsilon^{2}}{\varepsilon^{2}+\left(\frac{\partial \phi}{\partial x}\right)^{2}} \frac{\partial^{2} \phi}{\partial x^{2}}\left(\frac{\partial \phi}{\partial x}\right)^{20} \omega_{\varepsilon}^{3} d x \\
& \quad+3 \varepsilon \int_{0}^{l} \arctan \left(\frac{1}{\varepsilon} \frac{\partial \phi}{\partial x}\right)\left(\frac{\partial \phi}{\partial x}\right)^{20} \frac{\partial \omega_{\varepsilon}}{\partial x} \omega_{\varepsilon}^{2} d x .
\end{aligned}
$$

Thus, we have

$$
\begin{aligned}
& \frac{1}{4}\left\|\left(\frac{\partial \phi}{\partial x}\right)^{5} \omega_{\varepsilon}(., T)\right\|_{L^{4}(0, l)}^{4} \\
& =20 \varepsilon \int_{0}^{T} \int_{0}^{l}\left(\begin{array}{c}
\arctan \left(\frac{1}{\varepsilon} \frac{\partial \phi}{\partial x}\right)- \\
-\arctan \left(\frac{1}{\varepsilon} \frac{\partial u_{\varepsilon}}{\partial x}\right)
\end{array}\right) \frac{\partial^{2} \phi}{\partial x^{2}}\left(\frac{\partial \phi}{\partial x}\right)^{19} \omega_{\varepsilon}^{3} d x d t
\end{aligned}
$$




$$
\begin{aligned}
& -3 \varepsilon \int_{0}^{T} \int_{0}^{l}\left(\begin{array}{c}
\arctan \left(\frac{1}{\varepsilon} \frac{\partial u_{\varepsilon}}{\partial x}\right)- \\
-\arctan \left(\frac{1}{\varepsilon} \frac{\partial \phi}{\partial x}\right)
\end{array}\right)\left(\frac{\partial \phi}{\partial x}\right)^{20} \frac{\partial \omega_{\varepsilon}}{\partial x} \omega_{\varepsilon}^{2} d x d t \\
& +\int_{0}^{T} \int_{0}^{l} \frac{\varepsilon^{2}}{\varepsilon^{2}+\left(\frac{\partial \phi}{\partial x}\right)^{2}} \frac{\partial^{2} \phi}{\partial x^{2}}\left(\frac{\partial \phi}{\partial x}\right)^{20} \omega_{\varepsilon}^{3} d x d t .
\end{aligned}
$$

Noticing that

$$
\arctan \left(\frac{1}{\varepsilon} \frac{\partial u_{\varepsilon}}{\partial x}\right)-\arctan \left(\frac{1}{\varepsilon} \frac{\partial \phi}{\partial x}\right)=\arctan \left(\frac{1}{\varepsilon} \frac{1}{1+\frac{1}{\varepsilon^{2}} \frac{\partial \phi}{\partial x} \frac{\partial u_{\varepsilon}}{\partial x}} \frac{\partial \omega_{\varepsilon}}{\partial x}\right),
$$

we have

$$
\arctan \left(\frac{1}{\varepsilon} \frac{1}{1+\frac{1}{\varepsilon^{2}} \frac{\partial \phi}{\partial x} \frac{\partial u_{\varepsilon}}{\partial x}} \frac{\partial \omega_{\varepsilon}}{\partial x}\right) \frac{\partial \omega_{\varepsilon}}{\partial x} \geq 0
$$

and since $\frac{\partial \phi}{\partial x} \frac{\partial u_{\varepsilon}}{\partial x} \geq 0$ a.e., on $Q_{T}$, Lemma 5.52 ), we get

$$
3 \varepsilon \int_{0}^{T} \int_{0}^{l} \arctan \left(\frac{1}{\varepsilon} \frac{1}{1+\frac{1}{\varepsilon^{2}} \frac{\partial \phi}{\partial x} \frac{\partial u_{\varepsilon}}{\partial x}} \frac{\partial \omega_{\varepsilon}}{\partial x}\right) \frac{\partial \omega_{\varepsilon}}{\partial x}\left(\frac{\partial \phi}{\partial x}\right)^{20} \omega_{\varepsilon}^{2} d x d t \geq 0 .
$$

Thus, we obtain

$$
\begin{aligned}
\frac{1}{4} \| & \left(\frac{\partial \phi}{\partial x}\right)^{5} \omega_{\varepsilon}(., T) \|_{L^{4}(0, l)}^{4} \\
& +3 \varepsilon \int_{0}^{T} \int_{0}^{l} \arctan \left(\frac{1}{\varepsilon} \frac{1}{1+\frac{1}{\varepsilon^{2}} \frac{\partial \phi}{\partial x} \frac{\partial u_{\varepsilon}}{\partial x}} \frac{\partial \omega_{\varepsilon}}{\partial x}\right) \frac{\partial \omega_{\varepsilon}}{\partial x}\left(\frac{\partial \phi}{\partial x}\right)^{20} \omega_{\varepsilon}^{2} d x d t \\
\leq & 20 \varepsilon \int_{0}^{T} \int_{0}^{l}\left(\begin{array}{l}
\arctan \left(\frac{1}{\varepsilon} \frac{\partial \phi}{\partial x}\right)- \\
-\arctan \left(\frac{1}{\varepsilon} \frac{\partial u_{\varepsilon}}{\partial x}\right)
\end{array}\right) \frac{\partial^{2} \phi}{\partial x^{2}}\left(\frac{\partial \phi}{\partial x}\right)^{19} \omega_{\varepsilon}^{3} d x d t \\
& +\int_{0}^{T} \int_{0}^{l} \frac{\varepsilon^{2}}{\varepsilon^{2}+\left(\frac{\partial \phi}{\partial x}\right)^{2}} \frac{\partial^{2} \phi}{\partial x^{2}}\left(\frac{\partial \phi}{\partial x}\right)^{20} \omega_{\varepsilon}^{3} d x d t .
\end{aligned}
$$

Since $-\frac{\pi}{2} \leq \arctan x \leq \frac{\pi}{2}$, we estimate

$$
\begin{aligned}
& 20 \varepsilon\left|\int_{0}^{T} \int_{0}^{l}\left(\arctan \left(\frac{1}{\varepsilon} \frac{\partial \phi}{\partial x}\right)-\arctan \left(\frac{1}{\varepsilon} \frac{\partial u_{\varepsilon}}{\partial x}\right)\right) \frac{\partial^{2} \phi}{\partial x^{2}}\left(\frac{\partial \phi}{\partial x}\right)^{19} \omega_{\varepsilon}^{3} d x d t\right| \\
& \leq C_{1} \varepsilon^{5}+\int_{0}^{T}\left\|\left(\frac{\partial \phi}{\partial x}\right)^{5} \omega_{\varepsilon}\right\|_{L^{4}(0, l)}^{4} d t
\end{aligned}
$$


due to Lemma 5.115 ), and since $\frac{x^{2}}{\varepsilon^{2}+x^{2}} \leq 1$ for every real $x$, we have

$$
\begin{aligned}
& \left|\int_{0}^{T} \int_{0}^{l} \frac{\varepsilon^{2}}{\varepsilon^{2}+\left(\frac{\partial \phi}{\partial x}\right)^{2}}\left(\frac{\partial \phi}{\partial x}\right)^{20} \frac{\partial^{2} \phi}{\partial x^{2}} \omega_{\varepsilon}^{3} d x d t\right| \\
& \leq+\int_{0}^{T}\left\|\left(\frac{\partial \phi}{\partial x}\right)^{5} \omega_{\varepsilon}\right\|_{L^{4}(0, l)}^{4} d t+C_{2} \varepsilon^{6},
\end{aligned}
$$

due to Lemma 5.11 5). Finally, we obtain

$$
\frac{1}{4}\left\|\left(\frac{\partial \phi}{\partial x}\right)^{5} \omega_{\varepsilon}(., T)\right\|_{L^{4}(0, l)}^{4} \leq C_{3} \varepsilon^{5}+3 \int_{0}^{T}\left\|\left(\frac{\partial \phi}{\partial x}\right)^{5} \omega_{\varepsilon}\right\|_{L^{4}(0, l)}^{4} d t
$$

and we deduce, using Gronwall's Lemma, that $\left(\frac{1}{\varepsilon^{5 / 4}}\left(\frac{\partial \phi}{\partial x}\right)^{5} \omega_{\varepsilon}\right)_{\varepsilon}$ is bounded in $L^{4}\left(Q_{T}\right) \cap L^{\infty}\left(0, T ; L^{4}(0, l)\right)$.

3. 4. Setting $\varphi=\left(\frac{\partial \phi}{\partial x}\right)^{18}\left(\omega_{\varepsilon}\right)^{2} \frac{\partial \omega_{\varepsilon}}{\partial t}$ in (3.13), and using the same type of computations as in Lemma 5.73$)$, 4), we have

$$
\begin{array}{r}
\int_{0}^{T}\left\|\left(\frac{\partial \phi}{\partial x}\right)^{9} \omega_{\varepsilon} \frac{\partial \omega_{\varepsilon}}{\partial t}\right\|_{L^{2}(0, l)}^{2} \quad d t+\frac{1}{2 \varepsilon^{2}} \int_{0}^{T}\left\|\omega_{\varepsilon}\left(\frac{\partial \phi}{\partial x}\right)^{9} \frac{\partial u_{\varepsilon}}{\partial x} \frac{\partial \omega_{\varepsilon}}{\partial t}\right\|_{L^{2}(0, l)}^{2} d t \\
+\frac{1}{2}\left\|\left(\frac{\partial \phi}{\partial x}\right)^{9} \frac{\partial \omega_{\varepsilon}}{\partial x}(., T) \omega_{\varepsilon}(., T)\right\|_{L^{2}(0, l)}^{2} \leq C \varepsilon^{4} .
\end{array}
$$

This implies that $\left(\frac{1}{\varepsilon^{2}}\left(\frac{\partial \phi}{\partial x}\right)^{9} \frac{\partial \omega_{\varepsilon}}{\partial x} \omega_{\varepsilon}\right)_{\varepsilon}$ is bounded in $L^{\infty}\left(0, T ; L^{2}(0, l)\right) \cap$ $L^{2}\left(Q_{T}\right)$ and also that $\left(\frac{1}{\varepsilon^{2}}\left(\frac{\partial \phi}{\partial x}\right)^{9} \frac{\partial \omega_{\varepsilon}}{\partial t} \omega_{\varepsilon}\right)_{\varepsilon}$ is bounded in $L^{2}\left(Q_{T}\right)$.

5. 6. Using the same test-function $\varphi=\left(\frac{\partial \phi}{\partial x}\right)^{18} \omega_{\varepsilon}^{2} \frac{\partial \omega_{\varepsilon}}{\partial t}$ in (3.13), and using the same type of computations as in Lemma 5.73 ), 4), we obtain

$$
\begin{aligned}
& \int_{0}^{T}\left\|\left(\frac{\partial \phi}{\partial x}\right)^{9} \omega_{\varepsilon} \frac{\partial \omega_{\varepsilon}}{\partial t}\right\|_{L^{2}(0, l)}^{2} d t+\frac{1}{2 \varepsilon^{2}} \int_{0}^{T}\left\|\frac{\partial u_{\varepsilon}}{\partial x}\left(\frac{\partial \phi}{\partial x}\right)^{9} \omega_{\varepsilon} \frac{\partial \omega_{\varepsilon}}{\partial t}\right\|_{L^{2}(0, l)}^{2} d t \\
& +\frac{1}{4}\left\|\frac{\partial u_{\varepsilon}}{\partial x}(., T)\left(\frac{\partial \phi}{\partial x}\right)^{9} \omega_{\varepsilon}(., T)\right\|_{L^{2}(0, l)}^{2} \\
& \leq C_{1} \int_{0}^{T}\left\|\left(\frac{\partial \phi}{\partial x}\right)^{9} \omega_{\varepsilon} \frac{\partial u_{\varepsilon}}{\partial x}\right\|_{L^{2}(0, l)}^{2} d t+C_{0} \varepsilon^{4} .
\end{aligned}
$$


We deduce that $\left(\frac{1}{\varepsilon^{2}} \frac{\partial u_{\varepsilon}}{\partial x}\left(\frac{\partial \phi}{\partial x}\right)^{9} \omega_{\varepsilon}\right)_{\varepsilon}$ is bounded in $L^{\infty}\left(0, T ; L^{2}(0, l)\right) \cap$ $L^{2}\left(Q_{T}\right)$. Using the last estimate with Lemma 5.123 ), we deduce that $\left(\frac{1}{\varepsilon^{2}}\left(\frac{\partial \phi}{\partial x}\right)^{10} \omega_{\varepsilon}\right)_{\varepsilon}$ is bounded in $L^{\infty}\left(0, T ; L^{2}(0, l)\right) \cap L^{2}\left(Q_{T}\right)$.

Lemma 5.13. $\left(\frac{1}{\varepsilon} \frac{\partial \omega_{\varepsilon}}{\partial x}\left(\frac{\partial \phi}{\partial x}\right)^{5}\right)_{\varepsilon}$ is bounded in $L^{4}\left(Q_{T}\right)$.

Proof. We set $\varphi=\left(\frac{\partial \phi}{\partial x}\right)^{20}\left(\frac{\partial \omega_{\varepsilon}}{\partial x}\right)^{2} \omega_{\varepsilon}$ in (3.13) and we use the same type of computations as in Lemma 5.81 ) to obtain the result.

\section{Lemma 5.14.}

1) $\left(\frac{1}{\varepsilon^{2}}\left(\frac{\partial \phi}{\partial x}\right)^{11} \frac{\partial \omega_{\varepsilon}}{\partial x} \frac{\partial \omega_{\varepsilon}}{\partial t}\right)_{\varepsilon}$ is bounded in $L^{2}\left(Q_{T}\right)$.

2) $\left(\frac{1}{\varepsilon^{2}}\left(\frac{\partial \phi}{\partial x}\right)^{12} \frac{\partial \omega_{\varepsilon}}{\partial t}\right)_{\varepsilon}$ is bounded in $L^{2}\left(Q_{T}\right)$.

Proof.

1. We set $\varphi=\left(\frac{\partial \phi}{\partial x}\right)^{22} \frac{\partial \omega_{\varepsilon}}{\partial t}\left(\frac{\partial \omega_{\varepsilon}}{\partial x}\right)^{2}$ in (3.13) and compute

$$
\begin{aligned}
& \int_{0}^{T}\left\|\frac{\partial \omega_{\varepsilon}}{\partial x}\left(\frac{\partial \phi}{\partial x}\right)^{11} \frac{\partial \omega_{\varepsilon}}{\partial t}\right\|_{L^{2}(0, L)}^{2} d t+\frac{1}{\varepsilon^{2}} \int_{0}^{T}\left\|\frac{\partial u_{\varepsilon}}{\partial x} \frac{\partial \omega_{\varepsilon}}{\partial x}\left(\frac{\partial \phi}{\partial x}\right)^{11} \frac{\partial \omega_{\varepsilon}}{\partial t}\right\|_{L^{2}(0, L)}^{2} d t \\
& =-\frac{22}{3} \int_{0}^{T} \int_{0}^{l}\left(\frac{\partial \omega_{\varepsilon}}{\partial x}\right)^{3}\left(\frac{\partial \phi}{\partial x}\right)^{21} \frac{\partial^{2} \phi}{\partial x^{2}} \frac{\partial \omega_{\varepsilon}}{\partial t} d x d t \\
& \quad-\frac{1}{12} \int_{0}^{T} \int_{0}^{l} \frac{\partial}{\partial t}\left(\left(\frac{\partial \omega_{\varepsilon}}{\partial x}\right)^{4}\right)\left(\frac{\partial \phi}{\partial x}\right)^{22} d x d t \\
& +\int_{0}^{T} \int_{0}^{l} \frac{\partial^{2} \phi}{\partial x^{2}}\left(\frac{\partial \omega_{\varepsilon}}{\partial x}\right)^{2}\left(\frac{\partial \phi}{\partial x}\right)^{22} \frac{\partial \omega_{\varepsilon}}{\partial t} d x d t,
\end{aligned}
$$

which implies

$$
\begin{aligned}
\int_{0}^{T}\left\|\frac{\partial \omega_{\varepsilon}}{\partial x}\left(\frac{\partial \phi}{\partial x}\right)^{11} \frac{\partial \omega_{\varepsilon}}{\partial t}\right\|_{L^{2}(0, L)}^{2} d t+\frac{1}{\varepsilon^{2}} \int_{0}^{T}\left\|\frac{\partial u_{\varepsilon}}{\partial x} \frac{\partial \omega_{\varepsilon}}{\partial x}\left(\frac{\partial \phi}{\partial x}\right)^{11} \frac{\partial \omega_{\varepsilon}}{\partial t}\right\|_{L^{2}(0, L)}^{2} d t \\
=-\frac{19}{3} \int_{0}^{T} \int_{0}^{l}\left(\frac{\partial \omega_{\varepsilon}}{\partial x}\right)^{3}\left(\frac{\partial \phi}{\partial x}\right)^{21} \frac{\partial^{2} \phi}{\partial x^{2}} \frac{\partial \omega_{\varepsilon}}{\partial t} d x d t \\
\quad-\frac{1}{4} \int_{0}^{l}\left(\frac{\partial \omega_{\varepsilon}}{\partial x}\right)^{4}(x, T)\left(\frac{\partial \phi}{\partial x}\right)^{22}(x) d x \\
-\int_{0}^{T} \int_{0}^{l} \frac{\partial^{2} \phi}{\partial x^{2}}\left(\frac{\partial \omega_{\varepsilon}}{\partial x}\right)^{2}\left(\frac{\partial \phi}{\partial x}\right)^{21} \frac{\partial u_{\varepsilon}}{\partial x} \frac{\partial \omega_{\varepsilon}}{\partial t} d x d t,
\end{aligned}
$$


since $\frac{\partial \phi}{\partial x}=\frac{\partial u_{\varepsilon}}{\partial x}-\frac{\partial \omega_{\varepsilon}}{\partial x}$. We estimate the first term in the right hand side member of $(5.22)$

$$
\begin{aligned}
& \left|\frac{19}{3} \int_{0}^{T} \int_{0}^{l}\left(\frac{\partial \omega_{\varepsilon}}{\partial x}\right)^{3}\left(\frac{\partial \phi}{\partial x}\right)^{21} \frac{\partial^{2} \phi}{\partial x^{2}} \frac{\partial \omega_{\varepsilon}}{\partial t} d x d t\right| \\
& \leq C_{1} \varepsilon^{4}+\frac{1}{2} \int_{0}^{T}\left\|\left(\frac{\partial \phi}{\partial x}\right)^{11} \frac{\partial \omega_{\varepsilon}}{\partial x} \frac{\partial \omega_{\varepsilon}}{\partial t}\right\|_{L^{2}(0, L)}^{2} d t,
\end{aligned}
$$

due to Lemma 5.13. Then, having in mind Lemma 5.104 ), we estimate the fifth term of $(5.22)$

$$
\begin{aligned}
& \left|\int_{0}^{T} \int_{0}^{l} \frac{\partial^{2} \phi}{\partial x^{2}}\left(\frac{\partial \omega_{\varepsilon}}{\partial x}\right)^{2}\left(\frac{\partial \phi}{\partial x}\right)^{21} \frac{\partial u_{\varepsilon}}{\partial x} \frac{\partial \omega_{\varepsilon}}{\partial t} d x d t\right| \\
& \leq C_{2} \varepsilon^{4}+\frac{1}{2 \varepsilon^{2}} \int_{0}^{T}\left\|\left(\frac{\partial \phi}{\partial x}\right)^{11} \frac{\partial u_{\varepsilon}}{\partial x} \frac{\partial \omega_{\varepsilon}}{\partial x} \frac{\partial \omega_{\varepsilon}}{\partial t}\right\|_{L^{2}(0, L)}^{2} d t .
\end{aligned}
$$

Finally we get

$$
\begin{gathered}
\int_{0}^{T}\left\|\left(\frac{\partial \phi}{\partial x}\right)^{11} \frac{\partial \omega_{\varepsilon}}{\partial x} \frac{\partial \omega_{\varepsilon}}{\partial t}\right\|_{L^{2}(0, L)}^{2} d t+\frac{1}{\varepsilon^{2}} \int_{0}^{T}\left\|\left(\frac{\partial \phi}{\partial x}\right)^{11} \frac{\partial u_{\varepsilon}}{\partial x} \frac{\partial \omega_{\varepsilon}}{\partial x} \frac{\partial \omega_{\varepsilon}}{\partial t}\right\|_{L^{2}(0, L)}^{2} d t \\
+\frac{1}{6}\left\|\left(\frac{\partial \phi}{\partial x}\right)^{11}\left(\frac{\partial \omega_{\varepsilon}}{\partial x}\right)^{2}(., T)\right\|_{L^{2}(0, L)}^{2} d t \leq C_{3} \varepsilon^{4}
\end{gathered}
$$

which implies that $\left(\frac{1}{\varepsilon^{2}}\left(\frac{\partial \phi}{\partial x}\right)^{11} \frac{\partial \omega_{\varepsilon}}{\partial x} \frac{\partial \omega_{\varepsilon}}{\partial t}\right)_{\varepsilon}$ is bounded in $L^{2}\left(Q_{T}\right)$.

2. Using the above estimate and the boundedness of $\left(\frac{1}{\varepsilon^{2}}\left(\frac{\partial \phi}{\partial x}\right)^{11} \frac{\partial u_{\varepsilon}}{\partial x} \frac{\partial \omega_{\varepsilon}}{\partial t}\right)_{\varepsilon}$ in $L^{2}\left(Q_{T}\right)$, through Lemma 5.102$)$, since $\frac{\partial \phi}{\partial x}$ belongs to $L^{\infty}(0, l)$, we prove that the sequence $\left(\frac{1}{\varepsilon^{2}}\left(\frac{\partial \phi}{\partial x}\right)^{12} \frac{\partial \omega_{\varepsilon}}{\partial t}\right)_{\varepsilon}$ is bounded in $L^{2}\left(Q_{T}\right)$.

\subsection{The weak convergence.}

Theorem 5.15. The sequence $\left(\frac{1}{\varepsilon^{2}} \omega_{\varepsilon}\right)_{\varepsilon}$ converges weakly in $L^{2}\left(\left(\frac{\partial \phi}{\partial x}\right)^{12}\right.$, $\left.Q_{T}\right)$ to the solution $u_{2}$ of (3.10).

Proof. In Lemmas 5.14 2) and 5.12 6), we proved that the sequences $\left(\frac{1}{\varepsilon^{2}}\left(\frac{\partial \phi}{\partial x}\right)^{12} \frac{\partial \omega_{\varepsilon}}{\partial t}\right)_{\varepsilon}$ and $\left(\frac{1}{\varepsilon^{2}}\left(\frac{\partial \phi}{\partial x}\right)^{10} \omega_{\varepsilon}\right)_{\varepsilon}$ are bounded in $L^{\infty}\left(0, T ; L^{2}\left(Q_{T}\right)\right) \cap$ 
$L^{2}\left(Q_{T}\right)$. Hence $\left(\frac{1}{\varepsilon^{2}} \omega_{\varepsilon}\right)_{\varepsilon}$ and $\left(\frac{1}{\varepsilon^{2}} \frac{\partial \omega_{\varepsilon}}{\partial t}\right)_{\varepsilon}$ are bounded in $L^{2}\left(\left(\frac{\partial \phi}{\partial x}\right)^{12}, Q_{T}\right)$. Thus it is possible to prove the existence of $\chi$ in this space such that

$$
\begin{gathered}
\frac{1}{\varepsilon^{2}} \omega_{\varepsilon} \rightarrow_{\varepsilon \rightarrow 0} \chi \text { weakly in } L^{2}\left(\left(\frac{\partial \phi}{\partial x}\right)^{12}, Q_{T}\right) \\
\frac{1}{\varepsilon^{2}} \frac{\partial \omega_{\varepsilon}}{\partial t} \rightarrow_{\varepsilon \rightarrow 0} \frac{\partial \chi}{\partial t} \text { weakly in } L^{2}\left(\left(\frac{\partial \phi}{\partial x}\right)^{12}, Q_{T}\right) .
\end{gathered}
$$

From (3.12) we deduce that for $\varphi$ every in $C^{0}\left(0, T ; C^{1}(0, l)\right)$ we have

$$
\begin{aligned}
& \int_{0}^{T} \int_{0}^{l}\left(\frac{\partial \phi}{\partial x}\right)^{12} \frac{\partial \omega_{\varepsilon}}{\partial t} \varphi d x d t+\frac{1}{\varepsilon^{2}} \int_{0}^{T} \int_{0}^{l}\left(\frac{\partial \phi}{\partial x}\right)^{12}\left(\frac{\partial u_{\varepsilon}}{\partial x}\right)^{2} \frac{\partial \omega_{\varepsilon}}{\partial t} \varphi d x d t \\
& =\int_{0}^{T} \int_{0}^{l}\left(\frac{\partial \phi}{\partial x}\right)^{12} \frac{\partial^{2} u_{\varepsilon}}{\partial x^{2}} \varphi d x d t
\end{aligned}
$$

Since $\frac{\partial^{2} u_{\varepsilon}}{\partial x^{2}}=\frac{\partial^{2} \phi}{\partial x^{2}}+\frac{\partial^{2} \omega_{\varepsilon}}{\partial x^{2}}$ the right hand side term of (5.23) can be computed as

$$
\begin{aligned}
& \int_{0}^{T} \int_{0}^{l}\left(\frac{\partial \phi}{\partial x}\right)^{12} \frac{\partial^{2} u_{\varepsilon}}{\partial x^{2}} \varphi d x d t \\
& =-\int_{0}^{T} \int_{0}^{l}\left(\frac{\partial \phi}{\partial x}\right)^{12} \frac{\partial \omega_{\varepsilon}}{\partial x} \frac{\partial \varphi}{\partial x} d x d t-12 \int_{0}^{T} \int_{0}^{l} \frac{\partial^{2} \phi}{\partial x^{2}}\left(\frac{\partial \phi}{\partial x}\right)^{11} \frac{\partial \omega_{\varepsilon}}{\partial x} \varphi d x d t \\
& \quad+\int_{0}^{T} \int_{0}^{l}\left(\frac{\partial \phi}{\partial x}\right)^{12} \frac{\partial^{2} \phi}{\partial x^{2}} \varphi d x d t .
\end{aligned}
$$

Then using the equality

$$
\begin{aligned}
& \left(\frac{\partial u_{\varepsilon}}{\partial x}\right)^{2}=\left(\frac{\partial \phi}{\partial x}\right)^{2}+\left(\frac{\partial \omega_{\varepsilon}}{\partial x}\right)^{2}+2 \frac{\partial \omega_{\varepsilon}}{\partial x} \frac{\partial \phi}{\partial x} \\
& =\left(\frac{\partial \phi}{\partial x}\right)^{2}+\frac{\partial \omega_{\varepsilon}}{\partial x} \frac{\partial u_{\varepsilon}}{\partial x}+\frac{\partial \omega_{\varepsilon}}{\partial x} \frac{\partial \phi}{\partial x}
\end{aligned}
$$

the second term of (5.23) can be computed as

$$
\begin{aligned}
& \frac{1}{\varepsilon^{2}} \int_{0}^{T} \int_{0}^{l}\left(\frac{\partial \phi}{\partial x}\right)^{12}\left(\frac{\partial u_{\varepsilon}}{\partial x}\right)^{2} \frac{\partial \omega_{\varepsilon}}{\partial t} \varphi d x d t \\
& =\frac{1}{\varepsilon^{2}} \int_{0}^{T} \int_{0}^{l}\left(\frac{\partial \phi}{\partial x}\right)^{14} \frac{\partial \omega_{\varepsilon}}{\partial t} \varphi d x d t \\
& \quad+\frac{1}{\varepsilon^{2}} \int_{0}^{T} \int_{0}^{l} \frac{\partial \omega_{\varepsilon}}{\partial x} \frac{\partial u_{\varepsilon}}{\partial x}\left(\frac{\partial \phi}{\partial x}\right)^{12} \frac{\partial \omega_{\varepsilon}}{\partial t} \varphi d x d t
\end{aligned}
$$




$$
+\frac{1}{\varepsilon^{2}} \int_{0}^{T} \int_{0}^{l} \frac{\partial \omega_{\varepsilon}}{\partial x}\left(\frac{\partial \phi}{\partial x}\right)^{13} \frac{\partial \omega_{\varepsilon}}{\partial t} \varphi d x d t .
$$

Thus we obtain the following equality

$$
\begin{aligned}
& \int_{0}^{T} \int_{0}^{l}\left(\frac{\partial \phi}{\partial x}\right)^{12} \frac{\partial \omega_{\varepsilon}}{\partial t} \varphi d x d t+\frac{1}{\varepsilon^{2}} \int_{0}^{T} \int_{0}^{l}\left(\frac{\partial \phi}{\partial x}\right)^{14} \frac{\partial \omega_{\varepsilon}}{\partial t} \varphi d x d t \\
& \quad+\frac{1}{\varepsilon^{2}} \int_{0}^{T} \int_{0}^{l} \frac{\partial \omega_{\varepsilon}}{\partial x} \frac{\partial u_{\varepsilon}}{\partial x}\left(\frac{\partial \phi}{\partial x}\right)^{12} \frac{\partial \omega_{\varepsilon}}{\partial t} \varphi d x d t \\
& \quad+\frac{1}{\varepsilon^{2}} \int_{0}^{T} \int_{0}^{l} \frac{\partial \omega_{\varepsilon}}{\partial x}\left(\frac{\partial \phi}{\partial x}\right)^{13} \frac{\partial \omega_{\varepsilon}}{\partial t} \varphi d x d t \\
& =-\int_{0}^{T} \int_{0}^{l}\left(\frac{\partial \phi}{\partial x}\right)^{12} \frac{\partial \omega_{\varepsilon}}{\partial x} \frac{\partial \varphi}{\partial x} d x d t-12 \int_{0}^{T} \int_{0}^{l} \frac{\partial^{2} \phi}{\partial x^{2}}\left(\frac{\partial \phi}{\partial x}\right)^{11} \frac{\partial \omega_{\varepsilon}}{\partial x} \varphi d x d t \\
& \quad+\int_{0}^{T} \int_{0}^{l}\left(\frac{\partial \phi}{\partial x}\right)^{12} \frac{\partial^{2} \phi}{\partial x^{2}} \varphi d x d t .
\end{aligned}
$$

Using Lemmas 5.7 2), 5.10 2) and 5.14 2), we estimate

$$
\begin{aligned}
\left|\frac{1}{\varepsilon^{2}} \int_{0}^{T} \int_{0}^{l}\left(\frac{\partial \phi}{\partial x}\right)^{12} \frac{\partial \omega_{\varepsilon}}{\partial x} \frac{\partial u_{\varepsilon}}{\partial x} \frac{\partial \omega_{\varepsilon}}{\partial t} \varphi d x d t\right| & \leq C_{1} \varepsilon^{1 / 4}, \\
\left|\frac{1}{\varepsilon^{2}} \int_{0}^{T} \int_{0}^{l} \frac{\partial \omega_{\varepsilon}}{\partial x}\left(\frac{\partial \phi}{\partial x}\right)^{13} \frac{\partial \omega_{\varepsilon}}{\partial t} \varphi d x d t\right| & \leq C_{2} \varepsilon^{1 / 4},
\end{aligned}
$$

which implies

$$
\begin{gathered}
\int_{0}^{T} \int_{0}^{l}\left(\frac{\partial \phi}{\partial x}\right)^{12} \frac{\partial \omega_{\varepsilon}}{\partial t} \varphi d x d t \longrightarrow_{\varepsilon \rightarrow 0} 0 \\
\frac{1}{\varepsilon^{2}} \int_{0}^{T} \int_{0}^{l}\left(\frac{\partial \phi}{\partial x}\right)^{14} \frac{\partial \omega_{\varepsilon}}{\partial t} \varphi d x d t \longrightarrow_{\varepsilon \rightarrow 0} \int_{0}^{T} \int_{0}^{l}\left(\frac{\partial \phi}{\partial x}\right)^{14} \frac{\partial \chi}{\partial t} \varphi d x d t \\
\frac{1}{\varepsilon^{2}} \int_{0}^{T} \int_{0}^{l}\left(\frac{\partial \phi}{\partial x}\right)^{12} \frac{\partial \omega_{\varepsilon}}{\partial x} \frac{\partial u_{\varepsilon}}{\partial x} \frac{\partial \omega_{\varepsilon}}{\partial t} \varphi d x d t \longrightarrow_{\varepsilon \rightarrow 0} 0 \\
\frac{1}{\varepsilon^{2}} \int_{0}^{T} \int_{0}^{l} \frac{\partial \omega_{\varepsilon}}{\partial x}\left(\frac{\partial \phi}{\partial x}\right)^{13} \frac{\partial \omega_{\varepsilon}}{\partial t} \varphi d x d t \longrightarrow_{\varepsilon \rightarrow 0} 0 \\
-\int_{0}^{T} \int_{0}^{l}\left(\frac{\partial \phi}{\partial x}\right)^{12} \frac{\partial \omega_{\varepsilon}}{\partial x} \frac{\partial \varphi}{\partial x} d x d t \longrightarrow_{\varepsilon \rightarrow 0} 0 \\
-12 \int_{0}^{T} \int_{0}^{l} \frac{\partial^{2} \phi}{\partial x^{2}}\left(\frac{\partial \phi}{\partial x}\right)^{11} \frac{\partial \omega_{\varepsilon}}{\partial x} \varphi d x d t \longrightarrow{ }_{\varepsilon \rightarrow 0} 0 .
\end{gathered}
$$

Therefore, when $\varepsilon$ goes to 0 in (5.24), the above convergences imply 


$$
\begin{aligned}
\int_{0}^{T} \int_{0}^{l}\left(\frac{\partial \phi}{\partial x}\right)^{14} \frac{\partial \chi}{\partial t} \varphi d x d t=\int_{0}^{T} \int_{0}^{l}\left(\frac{\partial \phi}{\partial x}\right)^{12} & \frac{\partial^{2} \phi}{\partial x^{2}} \varphi d x d t \\
& \forall \varphi \in C^{0}\left(0, T ; C^{1}(0, l)\right) .
\end{aligned}
$$

Hence, by a density argument, this proves the convergence indicated in the Theorem.

5.3. Further estimates and strong convergence. In order to make sure that the asymptotic expansion (3.11) is available in $L^{2}\left(\left(\frac{\partial \phi}{\partial x}\right)^{p}, Q_{T}\right)$, for some exponent $p$, we define $\vartheta_{\varepsilon}=u_{\varepsilon}-\phi-\varepsilon^{2} u_{2}$, which satisfies the nonlinear problem

$$
\begin{aligned}
& \int_{0}^{T} \int_{0}^{l}\left(1+\frac{1}{\varepsilon^{2}}\left(\frac{\partial \vartheta_{\varepsilon}}{\partial x}+\frac{\partial \phi}{\partial x}+\varepsilon^{2} \frac{\partial u_{2}}{\partial x}\right)^{2}\right) \frac{\partial \vartheta_{\varepsilon}}{\partial t} \varphi d x d t \\
& \quad+\int_{0}^{T} \int_{0}^{l}\left(\varepsilon^{2}+\left(\frac{\partial \vartheta_{\varepsilon}}{\partial x}+\frac{\partial \phi}{\partial x}+\varepsilon^{2} \frac{\partial u_{2}}{\partial x}\right)^{2}\right) \frac{\partial u_{2}}{\partial t} \varphi d x d t \\
& \quad-\int_{0}^{T} \int_{0}^{l}\left(\frac{\partial^{2} \vartheta_{\varepsilon}}{\partial x^{2}}+\frac{\partial^{2} \phi}{\partial x^{2}}+\varepsilon^{2} \frac{\partial^{2} u_{2}}{\partial x^{2}}\right) \varphi d x d t=0, \quad \forall \varphi \in L^{2}\left(0, T ; H^{1}(0, l)\right) .
\end{aligned}
$$

Lemma 5.16. Suppose that $\phi$ belongs to $H^{4}(0, l)$. Then

1) $\left(\frac{1}{\varepsilon^{2}}\left(\frac{\partial \phi}{\partial x}\right)^{10} \vartheta_{\varepsilon}\right)$ is bounded in $L^{2}\left(Q_{T}\right) \cap L^{\infty}\left(0, T ; L^{2}(0, l)\right)$.

2) $\left(\frac{1}{\varepsilon}\left(\frac{\partial \phi}{\partial x}\right)^{5} \frac{\partial \vartheta_{\varepsilon}}{\partial x}\right)_{\varepsilon}$ is bounded in $L^{4}\left(Q_{T}\right)$.

3) $\left(\frac{1}{\varepsilon}\left(\frac{\partial \phi}{\partial x}\right)^{8} \frac{\partial \vartheta_{\varepsilon}}{\partial x}\right)_{\varepsilon}$ is bounded in $L^{2}\left(Q_{T}\right) \cap L^{\infty}\left(0, T ; L^{2}(0, l)\right)$.

4) $\left(\frac{1}{\varepsilon^{5 / 4}}\left(\frac{\partial \phi}{\partial x}\right)^{5} \vartheta_{\varepsilon}\right)_{\varepsilon}$ is bounded in $L^{4}\left(Q_{T}\right) \cap L^{\infty}\left(0, T ; L^{4}(0, l)\right)$.

Proof.

1. Using the fact that $\vartheta_{\varepsilon}=\omega_{\varepsilon}-\varepsilon^{2} u_{2}$ and that $\left(\frac{1}{\varepsilon^{2}}\left(\frac{\partial \phi}{\partial x}\right)^{10} \omega_{\varepsilon}\right)_{\varepsilon}$ is bounded in $L^{2}\left(Q_{T}\right) \cap L^{\infty}\left(0, T ; L^{2}(0, l)\right)$, Lemma 5.126$)$, we have

$$
\left\|\left(\frac{\partial \phi}{\partial x}\right)^{10} \vartheta_{\varepsilon}\right\|_{L^{2}\left(Q_{T}\right)} \leq\left\|\left(\frac{\partial \phi}{\partial x}\right)^{10} \omega_{\varepsilon}\right\|_{L^{2}\left(Q_{T}\right)}+\varepsilon^{2}\left\|\left(\frac{\partial \phi}{\partial x}\right)^{10} u_{2}\right\|_{L^{2}\left(Q_{T}\right)} .
$$

Since $\left(\frac{\partial \phi}{\partial x}\right)^{10} u_{2}$ belongs to $L^{2}\left(Q_{T}\right) \cap L^{\infty}\left(0, T ; L^{2}(0, l)\right)$, the last inequality proves the result. 
2. Since $\frac{\partial \vartheta_{\varepsilon}}{\partial x}=\frac{\partial \omega_{\varepsilon}}{\partial x}-\varepsilon^{2} \frac{\partial u_{2}}{\partial x}$ and $\left(\frac{1}{\varepsilon}\left(\frac{\partial \phi}{\partial x}\right)^{5} \frac{\partial \omega_{\varepsilon}}{\partial x}\right)_{\varepsilon}$ is bounded in $L^{4}\left(Q_{T}\right)$, Lemma 5.13, we have

$$
\left\|\left(\frac{\partial \phi}{\partial x}\right)^{5} \frac{\partial \vartheta_{\varepsilon}}{\partial x}\right\|_{L^{4}\left(Q_{T}\right)} \leq\left\|\left(\frac{\partial \phi}{\partial x}\right)^{5} \frac{\partial \omega_{\varepsilon}}{\partial x}\right\|_{L^{4}\left(Q_{T}\right)}+\varepsilon^{2}\left\|\left(\frac{\partial \phi}{\partial x}\right)^{5} \frac{\partial u_{2}}{\partial x}\right\|_{L^{4}\left(Q_{T}\right)},
$$

which implies the result.

3. Using a similar argument and the fact that $\left(\frac{1}{\varepsilon}\left(\frac{\partial \phi}{\partial x}\right)^{8} \frac{\partial \omega_{\varepsilon}}{\partial x}\right)_{\varepsilon}$ is bounded in $L^{2}\left(Q_{T}\right) \cap L^{\infty}\left(0, T ; L^{2}(0, l)\right)$, Lemma 5.104$)$. and assuming that $\left(\frac{\partial \phi}{\partial x}\right)^{8} \frac{\partial u_{2}}{\partial x}$ belongs to $L^{2}\left(Q_{T}\right) \cap L^{\infty}\left(0, T ; L^{2}(0, l)\right)$, we prove that $\left(\frac{1}{\varepsilon}\left(\frac{\partial \phi}{\partial x}\right)^{8} \frac{\partial \vartheta_{\varepsilon}}{\partial x}\right)_{\varepsilon}$ is bounded in $L^{2}\left(Q_{T}\right) \cap L^{\infty}\left(0, T ; L^{2}(0, l)\right)$.

4. Noticing that $\left(\frac{1}{\varepsilon^{5 / 4}}\left(\frac{\partial \phi}{\partial x}\right)^{5} \omega_{\varepsilon}\right)_{\varepsilon}$ is bounded in $L^{\infty}\left(0, T ; L^{4}(0, l)\right) \cap$ $L^{4}\left(Q_{T}\right)$, Lemma 5.122$)$ and writing

$$
\left\|\left(\frac{\partial \phi}{\partial x}\right)^{5} \vartheta_{\varepsilon}\right\|_{L^{4}\left(Q_{T}\right)} \leq\left\|\left(\frac{\partial \phi}{\partial x}\right)^{5} \omega_{\varepsilon}\right\|_{L^{4}\left(Q_{T}\right)}+\varepsilon^{2}\left\|\left(\frac{\partial \phi}{\partial x}\right)^{5} u_{2}\right\|_{L^{4}\left(Q_{T}\right)}
$$

we prove the last result, assuming that $\left(\frac{\partial \phi}{\partial x}\right)^{5} u_{2}$ belongs to $L^{4}\left(Q_{T}\right)$.

Lemma 5.17. Suppose that $\phi$ belongs to $H^{4}(0, l)$. Then

1) $\left(\frac{1}{\varepsilon^{2}}\left(\frac{\partial \phi}{\partial x}\right)^{13} \frac{\partial \vartheta_{\varepsilon}}{\partial x}\right)_{\varepsilon}$ is bounded in $L^{2}\left(Q_{T}\right) \cap L^{\infty}\left(0, T ; L^{2}(0, l)\right)$.

2) $\left(\frac{1}{\varepsilon^{2}}\left(\frac{\partial \phi}{\partial x}\right)^{13} \frac{\partial \vartheta_{\varepsilon}}{\partial t}\right)_{\varepsilon}$ is bounded in $L^{2}\left(Q_{T}\right)$.

3) $\left(\frac{1}{\varepsilon^{3}}\left(\frac{\partial \phi}{\partial x}\right)^{13} \frac{\partial u_{\varepsilon}}{\partial x} \frac{\partial \vartheta_{\varepsilon}}{\partial t}\right)_{\varepsilon}$ is bounded in $L^{2}\left(Q_{T}\right)$.

4) $\left(\frac{1}{\varepsilon^{2}}\left(\frac{\partial \phi}{\partial x}\right)^{13} \vartheta_{\varepsilon}\right)_{\varepsilon}$ is bounded in $L^{2}\left(Q_{T}\right) \cap L^{\infty}\left(0, T ; L^{2}(0, l)\right)$.

Proof.

1. 2. 3. We set $\varphi=\left(\frac{\partial \phi}{\partial x}\right)^{26} \frac{\partial \vartheta_{\varepsilon}}{\partial t}$ in $(5.25)$

$$
\begin{gathered}
\int_{0}^{T} \int_{0}^{l}\left(1+\frac{1}{\varepsilon^{2}}\left(\frac{\partial \vartheta_{\varepsilon}}{\partial x}+\frac{\partial \phi}{\partial x}+\varepsilon^{2} \frac{\partial u_{2}}{\partial x}\right)^{2}\right)\left(\frac{\partial \phi}{\partial x}\right)^{26}\left(\frac{\partial \vartheta_{\varepsilon}}{\partial t}\right)^{2} d x d t \\
-\int_{0}^{T} \int_{0}^{l}\left(\frac{\partial^{2} \vartheta_{\varepsilon}}{\partial x^{2}}+\frac{\partial^{2} \phi}{\partial x^{2}}+\varepsilon^{2} \frac{\partial^{2} u_{2}}{\partial x^{2}}\right)\left(\frac{\partial \phi}{\partial x}\right)^{26} \frac{\partial \vartheta_{\varepsilon}}{\partial t} d x d t
\end{gathered}
$$




$$
+\int_{0}^{T} \int_{0}^{l}\left(\varepsilon^{2}+\left(\frac{\partial \vartheta_{\varepsilon}}{\partial x}+\frac{\partial \phi}{\partial x}+\varepsilon^{2} \frac{\partial u_{2}}{\partial x}\right)^{2}\right) \frac{\partial u_{2}}{\partial t}\left(\frac{\partial \phi}{\partial x}\right)^{26} \frac{\partial \vartheta_{\varepsilon}}{\partial t} d x d t=0 .
$$

Using the fact that $u_{2}$ satisfies

$$
\int_{0}^{T} \int_{0}^{l}\left(\frac{\partial \phi}{\partial x}\right)^{14} \frac{\partial u_{2}}{\partial t} \varphi d x d t=\int_{0}^{T} \int_{0}^{l}\left(\frac{\partial \phi}{\partial x}\right)^{12} \frac{\partial^{2} \phi}{\partial x^{2}} \varphi d x d t
$$

for every $\varphi$ in $L^{2}\left(0, T ; H^{1}(0, l)\right)$, we simplify $(5.26)$ as

$$
\begin{aligned}
& \int_{0}^{T} \int_{0}^{l}\left(1+\frac{1}{\varepsilon^{2}}\left(\frac{\partial \vartheta_{\varepsilon}}{\partial x}+\frac{\partial \phi}{\partial x}+\varepsilon^{2} \frac{\partial u_{2}}{\partial x}\right)^{2}\right)\left(\frac{\partial \phi}{\partial x}\right)^{26}\left(\frac{\partial \vartheta_{\varepsilon}}{\partial t}\right)^{2} d x d t \\
& \quad+\int_{0}^{T} \int_{0}^{l}\left(\varepsilon^{2}+\left(\frac{\partial \vartheta_{\varepsilon}}{\partial x}+\varepsilon^{2} \frac{\partial u_{2}}{\partial x}\right) \frac{\partial u_{\varepsilon}}{\partial x}\right) \frac{\partial u_{2}}{\partial t}\left(\frac{\partial \phi}{\partial x}\right)^{26} \frac{\partial \vartheta_{\varepsilon}}{\partial t} d x d t \\
& \quad+\int_{0}^{T} \int_{0}^{l} \frac{\partial \phi}{\partial x}\left(\frac{\partial \vartheta_{\varepsilon}}{\partial x}+\varepsilon^{2} \frac{\partial u_{2}}{\partial x}\right) \frac{\partial u_{2}}{\partial t}\left(\frac{\partial \phi}{\partial x}\right)^{26} \frac{\partial \vartheta_{\varepsilon}}{\partial t} d x d t \\
& \quad-\int_{0}^{T} \int_{0}^{l}\left(\frac{\partial^{2} \vartheta_{\varepsilon}}{\partial x^{2}}+\varepsilon^{2} \frac{\partial^{2} u_{2}}{\partial x^{2}}\right)\left(\frac{\partial \phi}{\partial x}\right)^{26} \frac{\partial \vartheta_{\varepsilon}}{\partial t} d x d t=0 .
\end{aligned}
$$

We then integrate by parts the integral $\int_{0}^{T} \int_{0}^{l} \frac{\partial^{2} \vartheta_{\varepsilon}}{\partial x^{2}}\left(\frac{\partial \phi}{\partial x}\right)^{26} \frac{\partial \vartheta_{\varepsilon}}{\partial t} d x d t$ with respect to the $x$ variable

$$
\begin{aligned}
& \int_{0}^{T} \int_{0}^{l} \frac{\partial^{2} \vartheta_{\varepsilon}}{\partial x^{2}}\left(\frac{\partial \phi}{\partial x}\right)^{26} \frac{\partial \vartheta_{\varepsilon}}{\partial t} d x d t \\
& =-26 \int_{0}^{T} \int_{0}^{l} \frac{\partial \vartheta_{\varepsilon}}{\partial x}\left(\frac{\partial \phi}{\partial x}\right)^{25} \frac{\partial^{2} \phi}{\partial x^{2}} \frac{\partial \vartheta_{\varepsilon}}{\partial t} d x d t \\
& \quad-\frac{1}{2} \int_{0}^{l}\left(\frac{\partial \vartheta_{\varepsilon}}{\partial x}\right)^{2}(., T)\left(\frac{\partial \phi}{\partial x}\right)^{26} d x .
\end{aligned}
$$

Thus we obtain

$$
\begin{aligned}
& \int_{0}^{T}\left\|\left(\frac{\partial \phi}{\partial x}\right)^{13} \frac{\partial \vartheta_{\varepsilon}}{\partial t}\right\|_{L^{2}(0, l)}^{2} d t+\frac{1}{\varepsilon^{2}} \int_{0}^{T}\left\|\frac{\partial u_{\varepsilon}}{\partial x}\left(\frac{\partial \phi}{\partial x}\right)^{13} \frac{\partial \vartheta_{\varepsilon}}{\partial t}\right\|_{L^{2}(0, l)}^{2} d t \\
& +\frac{1}{2}\left\|\left(\frac{\partial \phi}{\partial x}\right)^{13} \frac{\partial \vartheta_{\varepsilon}}{\partial x}(., T)\right\|_{L^{2}(0, l)}^{2} \\
& =-\varepsilon^{2} \int_{0}^{T} \int_{0}^{l} \frac{\partial u_{2}}{\partial t}\left(\frac{\partial \phi}{\partial x}\right)^{26} \frac{\partial \vartheta_{\varepsilon}}{\partial t} d x d t \\
& \quad-\int_{0}^{T} \int_{0}^{l}\left(\frac{\partial \vartheta_{\varepsilon}}{\partial x}+\varepsilon^{2} \frac{\partial u_{2}}{\partial x}\right) \frac{\partial u_{\varepsilon}}{\partial x} \frac{\partial u_{2}}{\partial t}\left(\frac{\partial \phi}{\partial x}\right)^{26} \frac{\partial \vartheta_{\varepsilon}}{\partial t} d x d t
\end{aligned}
$$




$$
\begin{aligned}
& -\int_{0}^{T} \int_{0}^{l}\left(\frac{\partial \phi}{\partial x}\right)^{27}\left(\frac{\partial \vartheta_{\varepsilon}}{\partial x}+\varepsilon^{2} \frac{\partial u_{2}}{\partial x}\right) \frac{\partial u_{2}}{\partial t} \frac{\partial \vartheta_{\varepsilon}}{\partial t} d x d t \\
& -26 \int_{0}^{T} \int_{0}^{l}\left(\frac{\partial \phi}{\partial x}\right)^{25} \frac{\partial^{2} \phi}{\partial x^{2}} \frac{\partial \vartheta_{\varepsilon}}{\partial x} \frac{\partial \vartheta_{\varepsilon}}{\partial t} d x d t \\
& +\varepsilon^{2} \int_{0}^{T} \int_{0}^{l} \frac{\partial^{2} u_{2}}{\partial x^{2}}\left(\frac{\partial \phi}{\partial x}\right)^{26} \frac{\partial \vartheta_{\varepsilon}}{\partial t} d x d t .
\end{aligned}
$$

We estimate the different terms in the right-hand side of (5.27). The first term is estimated using (3.10) with $\varphi=\left(\frac{\partial \phi}{\partial x}\right)^{24} \frac{\partial \vartheta_{\varepsilon}}{\partial t}$, assuming that this function is smooth enough

$$
\begin{aligned}
& \varepsilon^{2}\left|\int_{0}^{T} \int_{0}^{l} \frac{\partial u_{2}}{\partial t}\left(\frac{\partial \phi}{\partial x}\right)^{26} \frac{\partial \vartheta_{\varepsilon}}{\partial t} d x d t\right| \\
& =\left|\int_{0}^{T} \int_{0}^{l} \varepsilon^{2} \frac{\partial^{2} \phi}{\partial x^{2}}\left(\frac{\partial \phi}{\partial x}\right)^{24} \frac{\partial \vartheta_{\varepsilon}}{\partial t} d x d t\right| \\
& \leq C_{1} \varepsilon^{4}+\frac{1}{4} \int_{0}^{T}\left\|\left(\frac{\partial \phi}{\partial x}\right)^{13} \frac{\partial \vartheta_{\varepsilon}}{\partial t}\right\|_{L^{2}(0, l)}^{2} d t .
\end{aligned}
$$

Since $\frac{\partial \vartheta_{\varepsilon}}{\partial x}+\varepsilon^{2} \frac{\partial u_{2}}{\partial x}=\frac{\partial u_{\varepsilon}}{\partial x}-\frac{\partial \phi}{\partial x}=\frac{\partial \omega_{\varepsilon}}{\partial x}$, we deduce the following estimate of the second term of (5.27) using once more (3.10) with $\varphi=\frac{\partial u_{\varepsilon}}{\partial x} \frac{\partial \omega_{\varepsilon}}{\partial x}\left(\frac{\partial \phi}{\partial x}\right)^{26} \frac{\partial \vartheta_{\varepsilon}}{\partial t}$, assuming this function is smooth enough

$$
\begin{aligned}
& \left|\int_{0}^{T} \int_{0}^{l} \frac{\partial u_{\varepsilon}}{\partial x}\left(\frac{\partial \vartheta_{\varepsilon}}{\partial x}+\varepsilon^{2} \frac{\partial u_{2}}{\partial x}\right)\left(\frac{\partial \phi}{\partial x}\right)^{26} \frac{\partial u_{2}}{\partial t} \frac{\partial \vartheta_{\varepsilon}}{\partial t} d x d t\right| \\
& =\left|\int_{0}^{T} \int_{0}^{l} \frac{\partial u_{\varepsilon}}{\partial x} \frac{\partial \omega_{\varepsilon}}{\partial x}\left(\frac{\partial \phi}{\partial x}\right)^{24} \frac{\partial^{2} \phi}{\partial x^{2}} \frac{\partial \vartheta_{\varepsilon}}{\partial t} d x d t\right| \\
& \leq \frac{1}{2 \varepsilon^{2}} \int_{0}^{T}\left\|\frac{\partial u_{\varepsilon}}{\partial x}\left(\frac{\partial \phi}{\partial x}\right)^{13} \frac{\partial \vartheta_{\varepsilon}}{\partial t}\right\|_{L^{2}(0, l)}^{2} d t+C_{2} \varepsilon^{4},
\end{aligned}
$$

since $\phi$ belongs to $H^{3}(0, l)$ and $\left(\frac{1}{\varepsilon}\left(\frac{\partial \phi}{\partial x}\right)^{8} \frac{\partial \omega_{\varepsilon}}{\partial x}\right)_{\varepsilon}$ is bounded in $L^{2}\left(Q_{T}\right)$, Lemma 5.10 4). The third and fourth terms of this right hand side member of (5.27) are computed in the following manner

$$
\begin{aligned}
& -26 \int_{0}^{T} \int_{0}^{l}\left(\frac{\partial \phi}{\partial x}\right)^{25} \frac{\partial^{2} \phi}{\partial x^{2}} \frac{\partial \vartheta_{\varepsilon}}{\partial x} \frac{\partial \vartheta_{\varepsilon}}{\partial t} d x d t \\
& -\int_{0}^{T} \int_{0}^{l}\left(\frac{\partial \phi}{\partial x}\right)^{27}\left(\frac{\partial \vartheta_{\varepsilon}}{\partial x}+\varepsilon^{2} \frac{\partial u_{2}}{\partial x}\right) \frac{\partial u_{2}}{\partial t} \frac{\partial \vartheta_{\varepsilon}}{\partial t} d x d t
\end{aligned}
$$




$$
\begin{aligned}
= & -27 \int_{0}^{T} \int_{0}^{l}\left(\frac{\partial \phi}{\partial x}\right)^{25} \frac{\partial^{2} \phi}{\partial x^{2}} \frac{\partial \vartheta_{\varepsilon}}{\partial x} \frac{\partial \vartheta_{\varepsilon}}{\partial t} d x d t \\
& -\varepsilon^{2} \int_{0}^{T} \int_{0}^{l}\left(\frac{\partial \phi}{\partial x}\right)^{25} \frac{\partial u_{2}}{\partial x} \frac{\partial^{2} \phi}{\partial x^{2}} \frac{\partial \vartheta_{\varepsilon}}{\partial t} d x d t
\end{aligned}
$$

due to (3.10) with $\varphi=\left(\frac{\partial \phi}{\partial x}\right)^{27}\left(\frac{\partial \vartheta_{\varepsilon}}{\partial x}+\varepsilon^{2} \frac{\partial u_{2}}{\partial x}\right) \frac{\partial \vartheta_{\varepsilon}}{\partial t}$. We observe that the last term of the right hand side member of (5.28) is trivially evaluated by

$$
\begin{aligned}
& \left|-\varepsilon^{2} \int_{0}^{T} \int_{0}^{l} \frac{\partial u_{2}}{\partial x}\left(\frac{\partial \phi}{\partial x}\right)^{25} \frac{\partial^{2} \phi}{\partial x^{2}} \frac{\partial \vartheta_{\varepsilon}}{\partial t} d x d t\right| \\
& \leq \frac{1}{4} \int_{0}^{T}\left\|\left(\frac{\partial \phi}{\partial x}\right)^{13} \frac{\partial \vartheta_{\varepsilon}}{\partial t}\right\|_{L^{2}(0, l)}^{2} d t+C_{2} \varepsilon^{4},
\end{aligned}
$$

since $\left(\frac{\partial \phi}{\partial x}\right)^{12} \frac{\partial u_{2}}{\partial x}$ belongs to $L^{2}\left(Q_{T}\right)$. Let us now estimate the first term of the right hand side member of (5.28)

$$
\begin{aligned}
& \left|27 \int_{0}^{T} \int_{0}^{l}\left(\frac{\partial \phi}{\partial x}\right)^{25} \frac{\partial^{2} \phi}{\partial x^{2}} \frac{\partial \vartheta_{\varepsilon}}{\partial x} \frac{\partial \vartheta_{\varepsilon}}{\partial t} d x d t\right| \\
& \leq \int_{0}^{T}\left\|\left(\frac{\partial \phi}{\partial x}\right)^{13} \frac{\partial \vartheta_{\varepsilon}}{\partial x}\right\|_{L^{2}(0, l)}^{2} d t+C_{3} \varepsilon^{4},
\end{aligned}
$$

since $\frac{\partial \vartheta_{\varepsilon}}{\partial t}=\frac{\partial \omega_{\varepsilon}}{\partial t}-\varepsilon^{2} \frac{\partial u_{2}}{\partial t},\left(\frac{1}{\varepsilon^{2}}\left(\frac{\partial \phi}{\partial x}\right)^{12} \frac{\partial \omega_{\varepsilon}}{\partial t}\right)_{\varepsilon}$ is bounded in $L^{2}\left(Q_{T}\right)$, Lemma 5.142 ) and assuming that $\left(\frac{\partial \phi}{\partial x}\right)^{12} \frac{\partial u_{2}}{\partial t}$ belongs to $L^{2}\left(Q_{T}\right)$. Then the fifth term of the right hand side member of (5.27) is evaluated in the following manner

$$
\begin{aligned}
& \varepsilon^{2}\left|\int_{0}^{T} \int_{0}^{l} \frac{\partial^{2} u_{2}}{\partial x^{2}}\left(\frac{\partial \phi}{\partial x}\right)^{26} \frac{\partial \vartheta_{\varepsilon}}{\partial t} d x d t\right| \\
& \leq C_{4} \varepsilon^{4}+\frac{1}{4} \int_{0}^{T}\left\|\left(\frac{\partial \phi}{\partial x}\right)^{13} \frac{\partial \vartheta_{\varepsilon}}{\partial t}\right\|_{L^{2}(0, l)}^{2} d t
\end{aligned}
$$

since the hypothesis on $\phi$ and (3.10) imply that $\frac{\partial^{2} u_{2}}{\partial x^{2}}$ belongs to $L^{2}\left(\left(\frac{\partial \phi}{\partial x}\right)^{13}\right.$, $\left.Q_{T}\right)$. Finally, we obtain

$$
\int_{0}^{T}\left\|\left(\frac{\partial \phi}{\partial x}\right)^{13} \frac{\partial \vartheta_{\varepsilon}}{\partial t}\right\|_{L^{2}(0, l)}^{2} d t+\frac{1}{\varepsilon^{2}} \int_{0}^{T}\left\|\left(\frac{\partial \phi}{\partial x}\right)^{13} \frac{\partial u_{\varepsilon}}{\partial x} \frac{\partial \vartheta_{\varepsilon}}{\partial t}\right\|_{L^{2}(0, l)}^{2} d t
$$


$+\left\|\left(\frac{\partial \phi}{\partial x}\right)^{13} \frac{\partial \vartheta_{\varepsilon}}{\partial x}(., T)\right\|_{L^{2}(0, l)}^{2} \leq C_{5} \varepsilon^{4}+2 \int_{0}^{T}\left\|\left(\frac{\partial \phi}{\partial x}\right)^{13} \frac{\partial \vartheta_{\varepsilon}}{\partial x}\right\|_{L^{2}(0, l)}^{2} d t$

Using Gronwall's Lemma, we deduce that the sequence $\left(\frac{1}{\varepsilon^{2}}\left(\frac{\partial \phi}{\partial x}\right)^{13} \frac{\partial \vartheta_{\varepsilon}}{\partial x}\right)_{\varepsilon}$ is bounded in $L^{2}\left(Q_{T}\right) \cap L^{\infty}\left(0, T ; L^{2}(0, l)\right), \quad\left(\frac{1}{\varepsilon^{2}}\left(\frac{\partial \phi}{\partial x}\right)^{13} \frac{\partial \vartheta_{\varepsilon}}{\partial t}\right)_{\varepsilon}$ and $\left(\frac{1}{\varepsilon^{3}}\left(\frac{\partial \phi}{\partial x}\right)^{13} \frac{\partial u_{\varepsilon}}{\partial x} \frac{\partial \vartheta_{\varepsilon}}{\partial t}\right)_{\varepsilon}$ are bounded in $L^{2}\left(Q_{T}\right)$.

4. This is a consequence of the boundedness of $\left(\frac{1}{\varepsilon^{2}}\left(\frac{\partial \phi}{\partial x}\right)^{13} \frac{\partial \vartheta_{\varepsilon}}{\partial t}\right)_{\varepsilon}$ in $L^{2}\left(Q_{T}\right)$, through a Cauchy-Schwarz inequality with respect to the time variable.

\section{Lemma 5.18.}

1) $\left(\frac{1}{\varepsilon^{17 / 4}} \frac{\partial u_{\varepsilon}}{\partial x}\left(\frac{\partial \phi}{\partial x}\right)^{13} \vartheta_{\varepsilon}\right)_{\varepsilon}$ is bounded in $L^{2}\left(Q_{T}\right) \cap L^{\infty}\left(0, T ; L^{2}(0, l)\right)$.

2) $\left(\frac{1}{\varepsilon^{17 / 4}}\left(\frac{\partial \phi}{\partial x}\right)^{13} \vartheta_{\varepsilon} \frac{\partial \vartheta_{\varepsilon}}{\partial t}\right)_{\varepsilon}$ is bounded in $L^{2}\left(Q_{T}\right)$.

Proof. Set $\varphi=\left(\frac{\partial \phi}{\partial x}\right)^{26} \vartheta_{\varepsilon}^{2} \frac{\partial \vartheta_{\varepsilon}}{\partial t}$ in (5.25). This leads to

$$
\begin{aligned}
\int_{0}^{T} & \left\|\left(\frac{\partial \phi}{\partial x}\right)^{13} \vartheta_{\varepsilon} \frac{\partial \vartheta_{\varepsilon}}{\partial t}\right\|_{L^{2}(0, l)}^{2} d t+\frac{1}{\varepsilon^{2}} \int_{0}^{T}\left\|\left(\frac{\partial \phi}{\partial x}\right)^{13} \frac{\partial u_{\varepsilon}}{\partial x} \vartheta_{\varepsilon} \frac{\partial \vartheta_{\varepsilon}}{\partial t}\right\|_{L^{2}(0, l)}^{2} d t \\
& +\int_{0}^{T} \int_{0}^{l}\left(\varepsilon^{2}+\left(\frac{\partial u_{\varepsilon}}{\partial x}\right)^{2}\right) \frac{\partial u_{2}}{\partial t}\left(\frac{\partial \phi}{\partial x}\right)^{26} \vartheta_{\varepsilon}^{2} \frac{\partial \vartheta_{\varepsilon}}{\partial t} d x d t \\
& -\int_{0}^{T} \int_{0}^{l} \frac{\partial^{2} u_{\varepsilon}}{\partial x^{2}}\left(\frac{\partial \phi}{\partial x}\right)^{26} \vartheta_{\varepsilon}^{2} \frac{\partial \vartheta_{\varepsilon}}{\partial t} d x d t=0 .
\end{aligned}
$$


We compute the fourth term of (5.29) as

$$
\begin{aligned}
- & \int_{0}^{T} \int_{0}^{l} \frac{\partial^{2} u_{\varepsilon}}{\partial x^{2}}\left(\frac{\partial \phi}{\partial x}\right)^{26} \vartheta_{\varepsilon}^{2} \frac{\partial \vartheta_{\varepsilon}}{\partial t} d x d t \\
= & 26 \int_{0}^{T} \int_{0}^{l} \frac{\partial u_{\varepsilon}}{\partial x}\left(\frac{\partial \phi}{\partial x}\right)^{25} \frac{\partial^{2} \phi}{\partial x^{2}} \vartheta_{\varepsilon}^{2} \frac{\partial \vartheta_{\varepsilon}}{\partial t} d x d t \\
& +2 \int_{0}^{T} \int_{0}^{l} \frac{\partial u_{\varepsilon}}{\partial x}\left(\frac{\partial \phi}{\partial x}\right)^{26} \frac{\partial \vartheta_{\varepsilon}}{\partial x} \vartheta_{\varepsilon} \frac{\partial \vartheta_{\varepsilon}}{\partial t} d x d t \\
& +\int_{0}^{T} \int_{0}^{l} \frac{\partial u_{\varepsilon}}{\partial x}\left(\frac{\partial \phi}{\partial x}\right)^{26} \vartheta_{\varepsilon}^{2} \frac{\partial^{2} \omega_{\varepsilon}}{\partial x \partial t} d x d t \\
& +\varepsilon^{2} \int_{0}^{T} \int_{0}^{l} \frac{\partial u_{\varepsilon}}{\partial x}\left(\frac{\partial \phi}{\partial x}\right)^{26} \vartheta_{\varepsilon}^{2} \frac{\partial^{2} u_{2}}{\partial x \partial t} d x d t \\
& -\int_{0}^{T}\left[\frac{\partial u_{\varepsilon}}{\partial x}\left(\frac{\partial \phi}{\partial x}\right)^{26} \vartheta_{\varepsilon}^{2} \frac{\partial \vartheta_{\varepsilon}}{\partial t}\right]_{0}^{l} d x d t .
\end{aligned}
$$

The second term of (5.30) is evaluated as

$$
26\left|\int_{0}^{T} \int_{0}^{l} \frac{\partial u_{\varepsilon}}{\partial x}\left(\frac{\partial \phi}{\partial x}\right)^{25} \frac{\partial^{2} \phi}{\partial x^{2}} \vartheta_{\varepsilon}^{2} \frac{\partial \vartheta_{\varepsilon}}{\partial t} d x d t\right| \leq C_{1} \varepsilon^{9 / 2},
$$

since $\left(\frac{1}{\varepsilon^{5 / 4}}\left(\frac{\partial \phi}{\partial x}\right)^{5} \vartheta_{\varepsilon}\right)_{\varepsilon}$ is bounded in $L^{4}\left(Q_{T}\right) \cap L^{\infty}\left(0, T ; L^{4}(0, l)\right)$, Lemma $5.164)$, and $\left(\frac{1}{\varepsilon^{2}}\left(\frac{\partial \phi}{\partial x}\right)^{13} \frac{\partial u_{\varepsilon}}{\partial x} \frac{\partial \vartheta_{\varepsilon}}{\partial t}\right)_{\varepsilon}$ is bounded in $L^{2}\left(Q_{T}\right)$, Lemma 5.173$)$. We now estimate the third term of (5.30)

$$
2\left|\int_{0}^{T} \int_{0}^{l} \frac{\partial u_{\varepsilon}}{\partial x}\left(\frac{\partial \phi}{\partial x}\right)^{26} \frac{\partial \vartheta_{\varepsilon}}{\partial x} \vartheta_{\varepsilon} \frac{\partial \vartheta_{\varepsilon}}{\partial t} d x d t\right| \leq C_{2} \varepsilon^{9 / 2},
$$

since $\left(\frac{1}{\varepsilon^{5 / 4}}\left(\frac{\partial \phi}{\partial x}\right)^{5} \vartheta_{\varepsilon}\right)_{\varepsilon}$ and $\left(\frac{1}{\varepsilon}\left(\frac{\partial \phi}{\partial x}\right)^{5} \frac{\partial \vartheta_{\varepsilon}}{\partial x}\right)_{\varepsilon}$ are bounded in $L^{4}\left(Q_{T}\right) \cap$ $L^{\infty}\left(0, T ; L^{4}(0, l)\right)$, Lemma 5.16 2), 4), and $\left(\frac{1}{\varepsilon^{3}}\left(\frac{\partial \phi}{\partial x}\right)^{13} \frac{\partial u_{\varepsilon}}{\partial x} \frac{\partial \vartheta_{\varepsilon}}{\partial t}\right)$ is bounded in $L^{2}\left(Q_{T}\right)$, Lemma 5.173$)$. We compute the fourth term of (5.30) integrating by parts and using the equality $\frac{\partial^{2} \omega_{\varepsilon}}{\partial x \partial t}=\frac{\partial^{2} u_{\varepsilon}}{\partial x \partial t}$

$$
\begin{aligned}
& \int_{0}^{T} \int_{0}^{l} \frac{\partial u_{\varepsilon}}{\partial x}\left(\frac{\partial \phi}{\partial x}\right)^{26} \vartheta_{\varepsilon}^{2} \frac{\partial^{2} \omega_{\varepsilon}}{\partial x \partial t} d x d t \\
& =\frac{1}{2}\left\|\frac{\partial u_{\varepsilon}}{\partial x}(., T)\left(\frac{\partial \phi}{\partial x}\right)^{13} \vartheta_{\varepsilon}(., T)\right\|_{L^{2}(0, l)}^{2}
\end{aligned}
$$




$$
-\int_{0}^{T} \int_{0}^{l}\left(\frac{\partial u_{\varepsilon}}{\partial x}\right)^{2}\left(\frac{\partial \phi}{\partial x}\right)^{26} \frac{\partial \vartheta_{\varepsilon}}{\partial t} \vartheta_{\varepsilon} d x d t
$$

The last term of the previous equality can be estimated as

$$
\begin{aligned}
&\left|\int_{0}^{T} \int_{0}^{l}\left(\frac{\partial u_{\varepsilon}}{\partial x}\right)^{2}\left(\frac{\partial \phi}{\partial x}\right)^{26} \vartheta_{\varepsilon} \frac{\partial \vartheta_{\varepsilon}}{\partial t} d x d t\right| \\
& \leq \int_{0}^{T}\left\|\left(\frac{\partial \phi}{\partial x}\right)^{13} \frac{\partial u_{\varepsilon}}{\partial x} \vartheta_{\varepsilon}\right\|_{L^{2}(0, l)}^{2} d t+C_{4} \varepsilon^{6},
\end{aligned}
$$

since $\left(\frac{1}{\varepsilon^{3}}\left(\frac{\partial \phi}{\partial x}\right)^{13} \frac{\partial u_{\varepsilon}}{\partial x} \frac{\partial \vartheta_{\varepsilon}}{\partial t}\right)_{\varepsilon}$ is bounded in $L^{2}\left(Q_{T}\right)$, Lemma 5.173$)$. The fifth term of (5.30) becomes

$$
\begin{aligned}
\varepsilon^{2}\left|\int_{0}^{T} \int_{0}^{l} \frac{\partial u_{\varepsilon}}{\partial x}\left(\frac{\partial \phi}{\partial x}\right)^{26} \vartheta_{\varepsilon}^{2} \frac{\partial^{2} u_{2}}{\partial x \partial t} d x d t\right| \\
\leq \int_{0}^{T}\left\|\left(\frac{\partial \phi}{\partial x}\right)^{13} \frac{\partial u_{\varepsilon}}{\partial x} \vartheta_{\varepsilon}\right\|_{L^{2}(0, l)}^{2} d t+C_{5} \varepsilon^{6},
\end{aligned}
$$

since $\left(\frac{1}{\varepsilon}\left(\frac{\partial \phi}{\partial x}\right)^{5} \vartheta_{\varepsilon}\right)_{\varepsilon}$ is bounded in $L^{2}\left(Q_{T}\right)$, Lemma 5.164$)$, and assuming that $\left(\frac{\partial \phi}{\partial x}\right)^{9} \frac{\partial^{2} u_{2}}{\partial x \partial t}$ belongs to $L^{\infty}\left(Q_{T}\right)$.

Using (3.10) with $\varphi=\left(\frac{\partial \phi}{\partial x}\right)^{24} \vartheta_{\varepsilon}^{2} \frac{\partial \vartheta_{\varepsilon}}{\partial t}$ in the first part of the fifth term of (5.29), we have

$$
\begin{aligned}
& \varepsilon^{2}\left|\int_{0}^{T} \int_{0}^{l} \frac{\partial u_{2}}{\partial t}\left(\frac{\partial \phi}{\partial x}\right)^{26} \vartheta_{\varepsilon}^{2} \frac{\partial \vartheta_{\varepsilon}}{\partial t} d x d t\right| \\
& =\varepsilon^{2}\left|\int_{0}^{T} \int_{0}^{l} \frac{\partial^{2} \phi}{\partial x^{2}}\left(\frac{\partial \phi}{\partial x}\right)^{24} \vartheta_{\varepsilon}^{2} \frac{\partial \vartheta_{\varepsilon}}{\partial t} d x d t\right| \\
& \leq \frac{1}{2} \int_{0}^{T}\left\|\left(\frac{\partial \phi}{\partial x}\right)^{13} \vartheta_{\varepsilon} \frac{\partial \vartheta_{\varepsilon}}{\partial t}\right\|_{L^{2}(0, l)}^{2} d t+C_{6} \varepsilon^{6},
\end{aligned}
$$

due to Lemma 5.161 ). Using (3.10) with $\varphi=\left(\frac{\partial u_{\varepsilon}}{\partial x}\right)^{2}\left(\frac{\partial \phi}{\partial x}\right)^{26} \vartheta_{\varepsilon}^{2} \frac{\partial \vartheta_{\varepsilon}}{\partial t}$ and the fact that $\vartheta_{\varepsilon}=\omega_{\varepsilon}-\varepsilon^{2} u_{2}$, we have in the second part of the fifth term of $(5.29)$

$$
\left|\int_{0}^{T} \int_{0}^{l}\left(\frac{\partial u_{\varepsilon}}{\partial x}\right)^{2} \frac{\partial u_{2}}{\partial t}\left(\frac{\partial \phi}{\partial x}\right)^{26} \vartheta_{\varepsilon}^{2} \frac{\partial \vartheta_{\varepsilon}}{\partial t} d x d t\right|
$$




$$
\begin{aligned}
& =\left|\int_{0}^{T} \int_{0}^{l}\left(\frac{\partial u_{\varepsilon}}{\partial x}\right)^{2} \frac{\partial^{2} \phi}{\partial x^{2}}\left(\frac{\partial \phi}{\partial x}\right)^{24} \vartheta_{\varepsilon}^{2} \frac{\partial \vartheta_{\varepsilon}}{\partial t} d x d t\right| \\
& \leq \frac{1}{2 \varepsilon^{2}} \int_{0}^{T}\left\|\left(\frac{\partial \phi}{\partial x}\right)^{13} \frac{\partial u_{\varepsilon}}{\partial x} \vartheta_{\varepsilon} \frac{\partial \vartheta_{\varepsilon}}{\partial t}\right\|_{L^{2}(0, l)}^{2} d t+C_{7} \varepsilon^{6},
\end{aligned}
$$

due to Lemma 5.12 5). The third term of (5.29) can be computed in the following way

$$
\begin{aligned}
- & \int_{0}^{T}\left[\frac{\partial u_{\varepsilon}}{\partial x}\left(\frac{\partial \phi}{\partial x}\right)^{26} \vartheta_{\varepsilon}^{2} \frac{\partial \vartheta_{\varepsilon}}{\partial t}\right]_{0}^{l} d x d t \\
= & -9 \int_{0}^{l} \frac{\partial^{2} \phi}{\partial x^{2}}\left(\frac{\partial \phi}{\partial x}\right)^{26}\left(\vartheta_{\varepsilon}\right)^{3}(., T) d x \\
& -3 \int_{0}^{l}\left(\frac{\partial \phi}{\partial x}\right)^{27} \frac{\partial \vartheta_{\varepsilon}}{\partial x}(., T)\left(\vartheta_{\varepsilon}\right)^{2}(., T) d x
\end{aligned}
$$

since $\frac{\partial u_{\varepsilon}}{\partial x}(0, t)=\frac{\partial \phi}{\partial x}(0), \frac{\partial u_{\varepsilon}}{\partial x}(l, t)=\frac{\partial \phi}{\partial x}(l)$ and $\frac{\partial \vartheta_{\varepsilon}}{\partial x}(x, 0)=0$. We then estimate

$$
\left|-9 \int_{0}^{l} \frac{\partial^{2} \phi}{\partial x^{2}}\left(\frac{\partial \phi}{\partial x}\right)^{26}\left(\vartheta_{\varepsilon}\right)^{3}(., T) d x\right| \leq C_{8} \varepsilon^{9 / 2},
$$

since $\left(\frac{1}{\varepsilon^{5 / 4}}\left(\frac{\partial \phi}{\partial x}\right)^{5} \vartheta_{\varepsilon}\right)_{\varepsilon}$ is bounded in $L^{\infty}\left(0, T ; L^{4}(0, l)\right)$, Lemma 5.164$)$, and $\left(\frac{1}{\varepsilon^{2}}\left(\frac{\partial \phi}{\partial x}\right)^{10} \vartheta_{\varepsilon}\right)_{\varepsilon}$ is bounded in $L^{\infty}\left(0, T ; L^{2}(0, l)\right)$, Lemma 5.161$)$. Then

$$
\left|3 \int_{0}^{l}\left(\frac{\partial \phi}{\partial x}\right)^{27}\left(\frac{\partial \vartheta_{\varepsilon}}{\partial x}\left(\vartheta_{\varepsilon}\right)^{2}\right)(., T) d x\right| \leq C_{9} \varepsilon^{9 / 2},
$$

since $\left(\frac{1}{\varepsilon^{5 / 4}}\left(\frac{\partial \phi}{\partial x}\right)^{5} \vartheta_{\varepsilon}\right)_{\varepsilon}$ is bounded in $L^{\infty}\left(0, T ; L^{4}(0, l)\right)$, Lemma 5.164$)$, and $\left(\frac{1}{\varepsilon^{2}}\left(\frac{\partial \phi}{\partial x}\right)^{13} \frac{\partial \vartheta_{\varepsilon}}{\partial x}\right)_{\varepsilon}$ is bounded in $L^{\infty}\left(0, T ; L^{2}(0, l)\right)$, Lemma 5.171$)$. Finally, we have

$$
\begin{aligned}
& \int_{0}^{T}\left\|\left(\frac{\partial \phi}{\partial x}\right)^{13} \vartheta_{\varepsilon} \frac{\partial \vartheta_{\varepsilon}}{\partial t}\right\|_{L^{2}(0, l)}^{2} d t+\frac{1}{\varepsilon^{2}} \int_{0}^{T}\left\|\left(\frac{\partial \phi}{\partial x}\right)^{13} \frac{\partial u_{\varepsilon}}{\partial x} \vartheta_{\varepsilon} \frac{\partial \vartheta_{\varepsilon}}{\partial t}\right\|_{L^{2}(0, l)}^{2} d t \\
& \quad+\left\|\frac{\partial u_{\varepsilon}}{\partial x}(., T)\left(\frac{\partial \phi}{\partial x}\right)^{13} \vartheta_{\varepsilon}(., T)\right\|_{L^{2}(0, l)}^{2}
\end{aligned}
$$




$$
\leq 4 \int_{0}^{T}\left\|\frac{\partial u_{\varepsilon}}{\partial x}\left(\frac{\partial \phi}{\partial x}\right)^{13} \vartheta_{\varepsilon}\right\|_{L^{2}(0, l)}^{2} d t+C_{10} \varepsilon^{9 / 2} .
$$

From Gronwall's Lemma, we deduce that $\left(\frac{1}{\varepsilon^{9 / 4}} \frac{\partial u_{\varepsilon}}{\partial x}\left(\frac{\partial \phi}{\partial x}\right)^{13} \vartheta_{\varepsilon}\right)_{\varepsilon}$ is bounded in $L^{2}\left(Q_{T}\right) \cap L^{\infty}\left(0, T ; L^{2}(0, l)\right)$. Moreover, $\left(\frac{1}{\varepsilon^{9 / 4}}\left(\frac{\partial \phi}{\partial x}\right)^{13} \vartheta_{\varepsilon} \frac{\partial \vartheta_{\varepsilon}}{\partial t}\right)_{\varepsilon}$ is bounded in $L^{2}\left(Q_{T}\right)$.

Lemma 5.19. $\left(\frac{1}{\varepsilon^{17 / 8}}\left(\frac{\partial \phi}{\partial x}\right)^{14} \vartheta_{\varepsilon}\right)_{\varepsilon}$ is bounded in $L^{2}\left(Q_{T}\right)$.

Proof. Set $\varphi=\left(\frac{\partial \phi}{\partial x}\right)^{26} \vartheta_{\varepsilon}^{2} \frac{\partial \vartheta_{\varepsilon}}{\partial t}$ in $(5.25)$

$$
\begin{aligned}
\int_{0}^{T} & \left\|\left(\frac{\partial \phi}{\partial x}\right)^{13} \vartheta_{\varepsilon} \frac{\partial \vartheta_{\varepsilon}}{\partial t}\right\|_{L^{2}(0, l)}^{2} d t+\frac{1}{\varepsilon^{2}} \int_{0}^{T}\left\|\left(\frac{\partial \phi}{\partial x}\right)^{13} \frac{\partial u_{\varepsilon}}{\partial x} \vartheta_{\varepsilon} \frac{\partial \vartheta_{\varepsilon}}{\partial t}\right\|_{L^{2}(0, l)}^{2} d t \\
& -\int_{0}^{T} \int_{0}^{l} \frac{\partial^{2} u_{\varepsilon}}{\partial x^{2}}\left(\frac{\partial \phi}{\partial x}\right)^{26} \vartheta_{\varepsilon}^{2} \frac{\partial \vartheta_{\varepsilon}}{\partial t} d x d t \\
& +\int_{0}^{T} \int_{0}^{l}\left(\varepsilon^{2}+\left(\frac{\partial u_{\varepsilon}}{\partial x}\right)^{2}\right) \frac{\partial u_{2}}{\partial t}\left(\frac{\partial \phi}{\partial x}\right)^{26} \vartheta_{\varepsilon}^{2} \frac{\partial \vartheta_{\varepsilon}}{\partial t} d x d t=0 .
\end{aligned}
$$

Using the equality $u_{\varepsilon}=\omega_{\varepsilon}+\phi$, the third term of (5.31) leads to

$$
\begin{aligned}
& -\int_{0}^{T} \int_{0}^{l} \frac{\partial^{2} u_{\varepsilon}}{\partial x^{2}}\left(\frac{\partial \phi}{\partial x}\right)^{26} \vartheta_{\varepsilon}^{2} \frac{\partial \vartheta_{\varepsilon}}{\partial t} d x d t \\
& =-\int_{0}^{T} \int_{0}^{l} \frac{\partial^{2} \omega_{\varepsilon}}{\partial x^{2}}\left(\frac{\partial \phi}{\partial x}\right)^{26} \vartheta_{\varepsilon}^{2} \frac{\partial \vartheta_{\varepsilon}}{\partial t} d x d t-\int_{0}^{T} \int_{0}^{l} \frac{\partial^{2} \phi}{\partial x^{2}}\left(\frac{\partial \phi}{\partial x}\right)^{26} \vartheta_{\varepsilon}^{2} \frac{\partial \vartheta_{\varepsilon}}{\partial t} d x d t .
\end{aligned}
$$

We estimate the last previous term as follows

$$
\left|\int_{0}^{T} \int_{0}^{l} \frac{\partial^{2} \phi}{\partial x^{2}}\left(\frac{\partial \phi}{\partial x}\right)^{26} \vartheta_{\varepsilon}^{2} \frac{\partial \vartheta_{\varepsilon}}{\partial t} d x d t\right| \leq C_{1} \varepsilon^{9 / 2},
$$

since $\left(\frac{1}{\varepsilon^{5 / 4}}\left(\frac{\partial \phi}{\partial x}\right)^{5} \vartheta_{\varepsilon}\right)_{\varepsilon}$ is bounded in $L^{4}\left(Q_{T}\right)$, Lemma 5.16 4) and $\left(\frac{1}{\varepsilon^{2}}\left(\frac{\partial \phi}{\partial x}\right)^{13} \frac{\partial \vartheta_{\varepsilon}}{\partial t}\right)_{\varepsilon}$ is bounded in $L^{2}\left(Q_{T}\right)$, Lemma 5.172$)$. Using the equality $\frac{\partial \vartheta_{\varepsilon}}{\partial t}=\frac{\partial \omega_{\varepsilon}}{\partial t}-\varepsilon^{2} \frac{\partial u_{2}}{\partial t}$, the first term of the right hand side member of (5.32) is evaluated as 


$$
\begin{aligned}
& -\int_{0}^{T} \int_{0}^{l} \frac{\partial^{2} \omega_{\varepsilon}}{\partial x^{2}}\left(\frac{\partial \phi}{\partial x}\right)^{26} \vartheta_{\varepsilon}^{2} \frac{\partial \vartheta_{\varepsilon}}{\partial t} d x d t \\
& =-\int_{0}^{T} \int_{0}^{l} \frac{\partial^{2} \omega_{\varepsilon}}{\partial x^{2}}\left(\frac{\partial \phi}{\partial x}\right)^{26} \vartheta_{\varepsilon}^{2} \frac{\partial \omega_{\varepsilon}}{\partial t} d x d t \\
& +\varepsilon^{2} \int_{0}^{T} \int_{0}^{l} \frac{\partial^{2} \omega_{\varepsilon}}{\partial x^{2}}\left(\frac{\partial \phi}{\partial x}\right)^{26} \vartheta_{\varepsilon}^{2} \frac{\partial u_{2}}{\partial t} d x d t .
\end{aligned}
$$

We compute the first term of (5.33)

$$
\begin{aligned}
- & \int_{0}^{T} \int_{0}^{l} \frac{\partial^{2} \omega_{\varepsilon}}{\partial x^{2}}\left(\frac{\partial \phi}{\partial x}\right)^{26} \vartheta_{\varepsilon}^{2} \frac{\partial \omega_{\varepsilon}}{\partial t} d x d t \\
= & 26 \int_{0}^{T} \int_{0}^{l} \frac{\partial \omega_{\varepsilon}}{\partial x}\left(\frac{\partial \phi}{\partial x}\right)^{25} \frac{\partial^{2} \phi}{\partial x^{2}} \vartheta_{\varepsilon}^{2} \frac{\partial \omega_{\varepsilon}}{\partial t} d x d t \\
& +2 \int_{0}^{T} \int_{0}^{l}\left(\frac{\partial \omega_{\varepsilon}}{\partial x}\right)^{2}\left(\frac{\partial \phi}{\partial x}\right)^{26} \vartheta_{\varepsilon} \frac{\partial \omega_{\varepsilon}}{\partial t} d x d t \\
& -2 \varepsilon^{2} \int_{0}^{T} \int_{0}^{l}\left(\frac{\partial \phi}{\partial x}\right)^{26} \vartheta_{\varepsilon} \frac{\partial u_{2}}{\partial x} \frac{\partial \omega_{\varepsilon}}{\partial t} d x d t \\
& -\int_{0}^{T} \int_{0}^{l}\left(\frac{\partial \omega_{\varepsilon}}{\partial x}\right)^{2}\left(\frac{\partial \phi}{\partial x}\right)^{26} \vartheta_{\varepsilon} \frac{\partial \vartheta_{\varepsilon}}{\partial t} d x d t \\
& +\frac{1}{2}\left\|\frac{\partial \omega_{\varepsilon}}{\partial x}(., T)\left(\frac{\partial \phi}{\partial x}\right)^{13} \vartheta_{\varepsilon}(., T)\right\|_{L^{2}(0, l)}^{2},
\end{aligned}
$$

since $\frac{\partial \vartheta_{\varepsilon}}{\partial x}=\frac{\partial \omega_{\varepsilon}}{\partial x}-\varepsilon^{2} \frac{\partial u_{2}}{\partial x}$. We estimate the second term of (5.34)

$$
\begin{aligned}
& \left|26 \int_{0}^{T} \int_{0}^{l} \frac{\partial \omega_{\varepsilon}}{\partial x}\left(\frac{\partial \phi}{\partial x}\right)^{25} \frac{\partial^{2} \phi}{\partial x^{2}} \vartheta_{\varepsilon}^{2} \frac{\partial \omega_{\varepsilon}}{\partial t} d x d t\right| \\
& \leq C \int_{0}^{T}\left\|\left(\frac{\partial \phi}{\partial x}\right)^{11} \frac{\partial \omega_{\varepsilon}}{\partial x} \frac{\partial \omega_{\varepsilon}}{\partial t}\right\|\left\|_{L^{2}(0, l)}\right\|\left(\frac{\partial \phi}{\partial x}\right)^{5} \vartheta_{\varepsilon} \|_{L^{4}(0, l)}^{2} d t \\
& \leq C_{2} \varepsilon^{9 / 2}
\end{aligned}
$$

since $\left(\frac{1}{\varepsilon^{2}}\left(\frac{\partial \phi}{\partial x}\right)^{11} \frac{\partial \omega_{\varepsilon}}{\partial x} \frac{\partial \omega_{\varepsilon}}{\partial t}\right)_{\varepsilon}$ is bounded in $L^{2}\left(Q_{T}\right)$ and $\left(\frac{1}{\varepsilon^{5 / 4}}\left(\frac{\partial \phi}{\partial x}\right)^{5} \vartheta_{\varepsilon}\right)_{\varepsilon}$ is bounded in $L^{4}\left(Q_{T}\right)$, Lemmas 5.141$\left.), 5.164\right)$. We estimate the third term and the fifth term of (5.34)

$$
2 \int_{0}^{T} \int_{0}^{l}\left(\frac{\partial \omega_{\varepsilon}}{\partial x}\right)^{2}\left(\frac{\partial \phi}{\partial x}\right)^{26} \vartheta_{\varepsilon} \frac{\partial \omega_{\varepsilon}}{\partial t} d x d t
$$




$$
\begin{aligned}
& -\int_{0}^{T} \int_{0}^{l}\left(\frac{\partial \omega_{\varepsilon}}{\partial x}\right)^{2}\left(\frac{\partial \phi}{\partial x}\right)^{26} \vartheta_{\varepsilon} \frac{\partial \vartheta_{\varepsilon}}{\partial t} d x d t \\
= & \int_{0}^{T} \int_{0}^{l}\left(\frac{\partial \omega_{\varepsilon}}{\partial x}\right)^{2}\left(\frac{\partial \phi}{\partial x}\right)^{26} \vartheta_{\varepsilon} \frac{\partial \vartheta_{\varepsilon}}{\partial t} d x d t \\
& +2 \varepsilon^{2} \int_{0}^{T} \int_{0}^{l}\left(\frac{\partial \omega_{\varepsilon}}{\partial x}\right)^{2}\left(\frac{\partial \phi}{\partial x}\right)^{26} \vartheta_{\varepsilon} \frac{\partial u_{2}}{\partial t} d x d t,
\end{aligned}
$$

since $\vartheta_{\varepsilon}=\omega_{\varepsilon}-\varepsilon^{2} u_{2}$. Then, using (3.10) with $\varphi=\left(\frac{\partial \phi}{\partial x}\right)^{26} \vartheta_{\varepsilon}\left(\frac{\partial \omega_{\varepsilon}}{\partial x}\right)^{2}$, the second term in the right hand side of (5.35) gives

$$
\begin{aligned}
2 \varepsilon^{2} \int_{0}^{T} \int_{0}^{l}\left(\frac{\partial \omega_{\varepsilon}}{\partial x}\right)^{2}\left(\frac{\partial \phi}{\partial x}\right)^{26} & \vartheta_{\varepsilon} \frac{\partial u_{2}}{\partial t} d x d t \\
& =2 \varepsilon^{2} \int_{0}^{T} \int_{0}^{l}\left(\frac{\partial \omega_{\varepsilon}}{\partial x}\right)^{2}\left(\frac{\partial \phi}{\partial x}\right)^{24} \frac{\partial^{2} \phi}{\partial x^{2}} \vartheta_{\varepsilon} d x d t
\end{aligned}
$$

and

$$
\begin{aligned}
& \left|2 \varepsilon^{2} \int_{0}^{T} \int_{0}^{l}\left(\frac{\partial \omega_{\varepsilon}}{\partial x}\right)^{2}\left(\frac{\partial \phi}{\partial x}\right)^{24} \frac{\partial^{2} \phi}{\partial x^{2}} \vartheta_{\varepsilon} d x d t\right| \\
& \leq C \varepsilon^{2} \int_{0}^{T}\left\|\left(\frac{\partial \phi}{\partial x}\right)^{5} \frac{\partial \omega_{\varepsilon}}{\partial x}\right\|_{L^{4}(0, l)}^{2}\left\|\left(\frac{\partial \phi}{\partial x}\right)^{10} \vartheta_{\varepsilon}\right\|_{L^{2}(0, l)} d t \\
& \leq C_{3} \varepsilon^{6},
\end{aligned}
$$

since $\left(\frac{1}{\varepsilon}\left(\frac{\partial \phi}{\partial x}\right)^{5} \frac{\partial \omega_{\varepsilon}}{\partial x}\right)_{\varepsilon}$ is bounded in $L^{4}\left(Q_{T}\right)$, Lemma 5.13, $\left(\frac{1}{\varepsilon^{2}}\left(\frac{\partial \phi}{\partial x}\right)^{10} \vartheta_{\varepsilon}\right)_{\varepsilon}$ is bounded in $L^{2}\left(Q_{T}\right)$, Lemma 5.161$)$. Using $\frac{\partial \omega_{\varepsilon}}{\partial x}=\frac{\partial u_{\varepsilon}}{\partial x}-\frac{\partial \phi}{\partial x}$, we estimate the first term in the right hand side of (5.35)

$$
\left|\int_{0}^{T} \int_{0}^{l}\left(\frac{\partial \omega_{\varepsilon}}{\partial x}\right)^{2}\left(\frac{\partial \phi}{\partial x}\right)^{26} \vartheta_{\varepsilon} \frac{\partial \vartheta_{\varepsilon}}{\partial t} d x d t\right| \leq C_{4} \varepsilon^{17 / 4},
$$

since $\left(\frac{1}{\varepsilon}\left(\frac{\partial \phi}{\partial x}\right)^{5} \frac{\partial \omega_{\varepsilon}}{\partial x}\right)$ is bounded in $L^{4}\left(Q_{T}\right)$, Lemma 5.13, $\left(\frac{1}{\varepsilon^{5 / 4}}\left(\frac{\partial \phi}{\partial x}\right)^{5} \vartheta_{\varepsilon}\right)$ is bounded in $L^{4}\left(Q_{T}\right) \cap L^{\infty}\left(0, T ; L^{4}(0, l)\right)$, Lemma 5.16 4), $\left(\frac{1}{\varepsilon^{2}}\left(\frac{\partial \phi}{\partial x}\right)^{13} \frac{\partial \vartheta_{\varepsilon}}{\partial t}\right)_{\varepsilon}$ and $\left(\frac{1}{\varepsilon^{3}}\left(\frac{\partial \phi}{\partial x}\right)^{13} \frac{\partial u_{\varepsilon}}{\partial x} \frac{\partial \vartheta_{\varepsilon}}{\partial t}\right)_{\varepsilon}$ are bounded in $L^{2}\left(Q_{T}\right)$, Lemma 5.172$\left.), 3\right)$. Then, we estimate the fourth term of (5.34)

$$
2 \varepsilon^{2}\left|\int_{0}^{T} \int_{0}^{l}\left(\frac{\partial \phi}{\partial x}\right)^{26} \vartheta_{\varepsilon} \frac{\partial u_{2}}{\partial x} \frac{\partial \omega_{\varepsilon}}{\partial x} \frac{\partial \omega_{\varepsilon}}{\partial t} d x d t\right| \leq C_{5} \varepsilon^{9 / 2},
$$


since $\left(\frac{1}{\varepsilon^{2}}\left(\frac{\partial \phi}{\partial x}\right)^{11} \frac{\partial \omega_{\varepsilon}}{\partial x} \frac{\partial \omega_{\varepsilon}}{\partial t}\right)_{\varepsilon}$ is bounded in $L^{2}\left(Q_{T}\right)$, Lemma 5.14 1), $\left(\frac{1}{\sqrt{\varepsilon}}\left(\frac{\partial \phi}{\partial x}\right)^{3} \vartheta_{\varepsilon}\right)_{\varepsilon}$ is bounded in $L^{4}\left(Q_{T}\right) \cap L^{\infty}\left(0, T ; L^{4}(0, l)\right)$, Lemma 5.9 1) and assuming $\left(\frac{\partial \phi}{\partial x}\right)^{12} \frac{\partial u_{2}}{\partial x}$ belongs to $L^{4}\left(Q_{T}\right)$. Finally we estimate the third term of (5.33) by setting $\varphi=\frac{\partial^{2} \omega_{\varepsilon}}{\partial x^{2}}\left(\frac{\partial \phi}{\partial x}\right)^{24} \vartheta_{\varepsilon}^{2}$ in (3.10) and integrating by parts with respect to $x$

$$
\begin{aligned}
\varepsilon^{2} \int_{0}^{T} \int_{0}^{l} \frac{\partial^{2} \omega_{\varepsilon}}{\partial x^{2}}\left(\frac{\partial \phi}{\partial x}\right)^{26} \vartheta_{\varepsilon}^{2} \frac{\partial u_{2}}{\partial t} d x d t \\
=\varepsilon^{2} \int_{0}^{T} \int_{0}^{l} \frac{\partial^{2} \omega_{\varepsilon}}{\partial x^{2}}\left(\frac{\partial \phi}{\partial x}\right)^{24} \vartheta_{\varepsilon}^{2} \frac{\partial^{2} \phi}{\partial x^{2}} d x d t \\
=-\varepsilon^{2}\left(\int_{0}^{T} \int_{0}^{l} \frac{\partial \omega_{\varepsilon}}{\partial x}\left(\frac{\partial \phi}{\partial x}\right)^{24} \vartheta_{\varepsilon}^{2} \frac{\partial^{3} \phi}{\partial x^{3}} d x d t\right. \\
+24 \int_{0}^{T} \int_{0}^{l} \frac{\partial \omega_{\varepsilon}}{\partial x}\left(\frac{\partial \phi}{\partial x}\right)^{23}\left(\frac{\partial^{2} \phi}{\partial x^{2}}\right)^{2} \vartheta_{\varepsilon}^{2} d x d t \\
\left.\quad+2 \int_{0}^{T} \int_{0}^{l} \frac{\partial \omega_{\varepsilon}}{\partial x}\left(\frac{\partial \phi}{\partial x}\right)^{24} \frac{\partial^{2} \phi}{\partial x^{2}} \vartheta_{\varepsilon} \frac{\partial \vartheta_{\varepsilon}}{\partial x} d x d t\right) .
\end{aligned}
$$

We estimate the different terms in the right hand side of the previous equality

$$
\begin{aligned}
& \left|-\varepsilon^{2} \int_{0}^{T} \int_{0}^{l} \frac{\partial \omega_{\varepsilon}}{\partial x}\left(\frac{\partial \phi}{\partial x}\right)^{24} \frac{\partial^{3} \phi}{\partial x^{3}} \vartheta_{\varepsilon}^{2} d x d t\right| \\
& \leq \int_{0}^{T}\left\|\left(\frac{\partial \phi}{\partial x}\right)^{13} \frac{\partial \omega_{\varepsilon}}{\partial x} \vartheta_{\varepsilon}\right\|_{L^{2}(0, l)}^{2} d t+C_{6} \varepsilon^{6}, \\
& \left|24 \varepsilon^{2} \int_{0}^{T} \int_{0}^{l} \frac{\partial \omega_{\varepsilon}}{\partial x}\left(\frac{\partial \phi}{\partial x}\right)^{23}\left(\frac{\partial^{2} \phi}{\partial x^{2}}\right)^{2} \vartheta_{\varepsilon}^{2} d x d t\right| \\
& \leq \int_{0}^{T}\left\|\left(\frac{\partial \phi}{\partial x}\right)^{13} \frac{\partial \omega_{\varepsilon}}{\partial x} \vartheta_{\varepsilon}\right\|_{L^{2}(0, l)}^{2} d t+C_{7} \varepsilon^{6} \\
& \left|2 \varepsilon^{2} \int_{0}^{T} \int_{0}^{l} \frac{\partial \omega_{\varepsilon}}{\partial x}\left(\frac{\partial \phi}{\partial x}\right)^{24} \frac{\partial^{2} \phi}{\partial x^{2}} \vartheta_{\varepsilon} \frac{\partial \vartheta_{\varepsilon}}{\partial x} d x d t\right| \\
& \leq \int_{0}^{T}\left\|\left(\frac{\partial \phi}{\partial x}\right)^{13} \frac{\partial \omega_{\varepsilon}}{\partial x} \vartheta_{\varepsilon}\right\|_{L^{2}(0, l)}^{2} d t+C_{8} \varepsilon^{6},
\end{aligned}
$$


due to Lemma 5.16 3). Then, we estimate the fourth term of (5.31)

$$
\begin{aligned}
\left|\int_{0}^{T} \int_{0}^{l}\left(\varepsilon^{2}+\left(\frac{\partial u_{\varepsilon}}{\partial x}\right)^{2}\right) \frac{\partial u_{2}}{\partial t}\left(\frac{\partial \phi}{\partial x}\right)^{26} \vartheta_{\varepsilon}^{2} \frac{\partial \vartheta_{\varepsilon}}{\partial t} d x d t\right| \\
=\mid \varepsilon^{2} \int_{0}^{T} \int_{0}^{l} \frac{\partial^{2} \phi}{\partial x^{2}}\left(\frac{\partial \phi}{\partial x}\right)^{24} \vartheta_{\varepsilon}^{2} \frac{\partial \vartheta_{\varepsilon}}{\partial t} d x d t \\
\quad+\int_{0}^{T} \int_{0}^{l}\left(\frac{\partial u_{\varepsilon}}{\partial x}\right)^{2} \frac{\partial^{2} \phi}{\partial x^{2}}\left(\frac{\partial \phi}{\partial x}\right)^{24} \vartheta_{\varepsilon}^{2} \frac{\partial \vartheta_{\varepsilon}}{\partial t} d x d t \mid \\
\leq \frac{1}{4 \varepsilon^{2}} \int_{0}^{T}\left\|\left(\frac{\partial \phi}{\partial x}\right)^{13} \frac{\partial u_{\varepsilon}}{\partial x} \vartheta_{\varepsilon} \frac{\partial \vartheta_{\varepsilon}}{\partial t}\right\|_{L^{2}(0, l)}^{2} d t+C_{9} \varepsilon^{6},
\end{aligned}
$$

due to Lemma 5.16 1). Finally, we have

$$
\begin{aligned}
& \frac{1}{4} \int_{0}^{T}\left\|\left(\frac{\partial \phi}{\partial x}\right)^{13} \vartheta_{\varepsilon} \frac{\partial \vartheta_{\varepsilon}}{\partial t}\right\|_{L^{2}(0, l)}^{2} d t+\frac{1}{4 \varepsilon^{2}} \int_{0}^{T}\left\|\left(\frac{\partial \phi}{\partial x}\right)^{13} \frac{\partial u_{\varepsilon}}{\partial x} \vartheta_{\varepsilon} \frac{\partial \vartheta_{\varepsilon}}{\partial t}\right\|_{L^{2}(0, l)}^{2} d t \\
& \quad+\frac{1}{2}\left\|\frac{\partial \omega_{\varepsilon}}{\partial x}\left(\frac{\partial \phi}{\partial x}\right)^{13} \vartheta_{\varepsilon}(., T)\right\|_{L^{2}(0, l)}^{2} \\
& \leq C \int_{0}^{T}\left\|\left(\frac{\partial \phi}{\partial x}\right)^{13} \frac{\partial \omega_{\varepsilon}}{\partial x} \vartheta_{\varepsilon}\right\|_{L^{2}(0, l)}^{2} d t+C_{10} \varepsilon^{17 / 4} .
\end{aligned}
$$

From Gronwall's Lemma, we deduce that $\left(\frac{1}{\varepsilon^{17 / 8}}\left(\frac{\partial \phi}{\partial x}\right)^{13} \frac{\partial \omega_{\varepsilon}}{\partial x} \vartheta_{\varepsilon}\right)_{\varepsilon}$ is bounded in $L^{2}\left(Q_{T}\right) \cap L^{\infty}\left(0, T ; L^{2}(0, l)\right)$. Using the boundedness of $\left(\frac{1}{\varepsilon^{9 / 4}}\left(\frac{\partial \phi}{\partial x}\right)^{13} \frac{\partial u_{\varepsilon}}{\partial x} \vartheta_{\varepsilon}\right)_{\varepsilon}$ in $L^{2}\left(Q_{T}\right) \cap L^{\infty}\left(0, T ; L^{2}(0, l)\right)$, Lemma 5.182$)$, we deduce the result.

We thus conclude with the following:

Theorem 5.20. Assume that $\phi$ belongs to $H^{4}(0, l)$. Then:

1) The asymptotic expansion (3.11) is available in $L^{2}\left(\left(\frac{\partial \phi}{\partial x}\right)^{14}, Q_{T}\right)$.

2) $\left(\frac{1}{\varepsilon^{2}} \omega_{\varepsilon}\right)_{\varepsilon}$ converges strongly to $u_{2}$ in $L^{2}\left(\left(\frac{\partial \phi}{\partial x}\right)^{14}, Q_{T}\right)$. 
Remark 5.21. The previous results are available for the anisotropic mean curvature flow $[\mathbf{D}]$. The extension of these results to higher dimension will be developed in a forthcoming paper.

\section{References}

[BSS] G. Barles, H.M. Soner and P.E. Souganidis, Front propagation and phase field theory, SIAM J. Control Optim., 31(2) (1993), 439-469.

[CGG] Y.G. Chen, Y. Giga and S. Goto, Uniqueness and existence of viscosity solutions of generalized mean curvature flow equation, J. of Diff. Geometry, 33(3) (1991), 749-786.

[CLI] M.G. Crandall, H. Ishii and P.L. Lions, User's guide to viscosity solutions of second order partial differential equations, Bull. AMS, 27(1) (1992), 1-67.

[DD] K. Deckelnick and G. Dziuk, Convergence of a finite element method for nonparametric mean curvtaure flow, Numerische Mathematik, 72(2) (1995), 197-222.

[D] Y. Dumont, Theoretical studies on the anisotropic curve shortening and the $\varepsilon$ regularized Mean Curvature Flow, PhD, 1998, Mulhouse-France.

[D99] , The E-regularized mean curvature flow in one dimension space, Journal of Computational Analysis and Applications, 2(1) (2000), 11-47.

[EH] K. Ecker and G. Huisken, Mean curvature evolution of entire graphs, Annals of Math., 130(3) (1989), 453-471.

[ES1] L.C. Evans and J. Spruck, Motion of level sets by mean curvature I, J. of Diff. Geometry, 33(3) (1991), 635-681.

[ES2] _ Motion of level sets by mean curvature II, Trans. Amer. Math. Soc., 330(1) (1992), 321-332.

[ES3] _ Motion of level sets by mean curvature III, J. Geom. Anal., 2 (1992), 121-150.

[ES4] Motion of level sets by mean curvature IV, J. Geom. Anal., 5(1) (1995), $77-114$.

[ESS] L.C. Evans, H.M. Soner and P.E. Souganidis, Phase transitions and generalized motion by mean curvature, Comm. in Pure and Applied Math., 45(9) (1992), 1097-1123.

[Fr] M. Fried, Berechnung des Krummungsflusses von Niveauflachen, Diplomarbeit, Freiburg, 1993.

[F] A. Friedman, Partial Differential Equations of Parabolic type, Prentice-Hall, (1964), 347 pp.

[LSU] O.A. Ladyzenskaya, V.A. Solonnikov and N.N. Ural'ceva, Linear and Quasilinear Equations of Parabolic-type, Translations of Mathematical Monographs, Vol. 23, A.M.S., (1967), 648 pp.

[SZ] P. Sternberg and W. Ziemer, Generalized motion by curvature with a Dirichlet condition, J. of Diff. Equations, 114(2) (1994), 580-600. 
[W] U. Wandtke, Ein finite element Algorithmus fuer den mittleren Kruemmunsgsfluss, Diplomarbeit, Bonn, 1991.

Received April 9, 1999. This paper is dedicated to my son Axel.

\section{IREMIA - UNIVERSITÉ DE LA REUNION}

15 AVEnUe Rene CASSIN

B.P 7151

97715 Saint Denis Messag, Cedex 9

ILE DE LA REUNION

FRANCE

E-mail address: Yves.Dumont@univ-reunion.fr 